\title{
Geometria de curvas e subvariedades bi-harmônicas
}

Apoenã Passos Passamani 

Data de Depósito:

Assinatura:

\section{Apoenã Passos Passamani}

\section{Geometria de curvas e subvariedades bi-harmônicas}

Tese apresentada ao Instituto de Ciências Matemáticas e de Computação - ICMC-USP, como parte dos requisitos para obtenção do título de Doutor em Ciências - Matemática. VERSÃO REVISADA

Área de Concentração: Matemática

Orientadora: Profa. Dra. Irene Ignazia Onnis

Coorientador: Prof. Dr. Stefano Montaldo 
Ficha catalográfica elaborada pela Biblioteca Prof. Achille Bassi e Seção Técnica de Informática, ICMC/USP, com os dados fornecidos pelo(a) autor(a)

P289g Passos Passamani, Apoenã
Geometria de curvas e subvariedades bi-harmônicas
/ Apoenã Passos Passamani; orientador Irene I.
Onnis; co-orientador Stefano Montaldo. -- São
Carlos, 2015.
109 p.
Tese (Doutorado - Programa de Pós-Graduação em
Matemática) -- Instituto de Ciências Matemáticas e
de Computação, Universidade de São Paulo, 2015.
1. Imersões bi-harmônicas. 2. Imersões
biconservativas. 3. Superfícies com aplicação de
Gauss bi-harmônica. 4. Superfícies de ângulo
constante. I. Onnis, Irene I., orient. II.
Montaldo, Stefano, co-orient. III. Título.




\section{Apoenã Passos Passamani}

\section{Geometry of biharmonic curves and submanifolds}

Doctoral dissertation submitted to the Instituto de Ciências Matemáticas e de Computação - ICMCUSP, in partial fulfillment of the requirements for the degree of the Doctorate Program in Mathematics. FINAL VERSION

Concentration Area: Mathematics

Advisor: Profa. Dra. Irene Ignazia Onnis Coadvisor: Prof. Dr. Stefano Montaldo

USP - São Carlos

August 2015 

À minha amada avó

Zilda 



\section{Agradecimentos}

A Deus, por seu cuidado me dando saúde e forças para concluir essa etapa de minha vida e também por ter colocado pessoas tão especias nesta minha caminhada. Agradeço a Deus também pelas oportunidades de crescimento e aprendizagem das quais este doutorado faz parte.

À minha amada família que sempre me apoiou e me deu muitas felicidades e orgulho. Em particular ao meu pai, que acumula as funções de melhor amigo e conselheiro, e à minha mãe guerreira e grande exemplo de perseverança. Quero também citar meus avós Carlos, Guaraci, Leonilde e Zilda, por sua grande influência positiva em minha educação. E não posso deixar de agradecer às minhas irmãs Tainah, Taiana e Fernanda, por serem amigas e grande motivo de felicidade que me incentiva diariamente.

À minha namorada Ginnara, que me apoiou, foi compreensiva e me ajudou no dia a dia desta conquista.

Aos meus amigos, todos com sua importância, que foram fundamentais nesta etapa de minha vida longe de minha família, muitas vezes tendo feito as vezes desta. Em especial a Alcebíades, Alex, Natália, Andreza, Mayron, Bruna, Camila, Flávio, Nelson, dona Wilma e Sr. Luís, Northon, Patrícia, Thaís Maria, Rafael Borro, Alexandra, Rafael Morais, Vinícius. 
À minha orientadora profa. Irene Ignazia Onnis por ter sido atenciosa, dedicada e incentivadora.

Ao professor Stefano Montaldo por ter me recebido de forma calorosa em meu estágio na Itália e ter se dedicado na coorientação de minha tese.

Por fim, agradeço a Capes pelo suporte financeiro. 
Neste trabalho estudamos essencialmente problemas relacionados aos conceitos de superfícies e curvas bi-harmônicas e de superfícies de ângulo constante.

Caracterizamos as curva bi-harmônicas do grupo especial linear $\operatorname{SL}(2, \mathbb{R})$. Em particular, mostramos que todas as curvas bi-harmônicas de $\operatorname{SL}(2, \mathbb{R})$ são hélices e damos suas parametrizações explícitas como curvas do espaço pseudo-Euclidiano $\mathbb{R}_{2}^{4}$.

Estudamos as superfícies biconservativas (as quais representam uma grande família que inclui as superfícies bi-harmônicas) nos espaços de Bianchi-Cartan-Vranceanu, obtendo a caracterização daquelas de ângulo constante e daquelas $\mathrm{SO}(2)$-invariantes. Também, caracterizamos as superfícies de ângulo constante do espaço Euclidiano tridimensional que possuem aplicação de Gauss bi-harmônica, provando que são cilindros de Hopf sobre uma clotóide.

Além disto, caracterizamos as superfícies de ângulo contante de $\operatorname{SL}(2, \mathbb{R})$. Mais especificamente, damos uma descrição local explícita para estas superfícies em termos de uma determinada curva de $\mathrm{SL}(2, \mathbb{R})$ e de uma família a um parâmetro de isometrias do espaço ambiente. 
Palavras chave: imersões bi-harmônicas, imersões biconservativas, superfícies com aplicação de Gauss bi-harmônica, superfícies de ângulo constante. 
In this work we mainly study some problems related to the concept of biharmonic curves and surfaces and to surfaces of constant angle.

We characterize the biharmonic curves in the special linear group $\operatorname{SL}(2, \mathbb{R})$. In particular, we show that all proper biharmonic curves in $\operatorname{SL}(2, \mathbb{R})$ are helices and we give their explicit parametrizations as curves in the pseudo-Euclidean space $\mathbb{R}_{2}^{4}$.

We study the biconservative surfaces (which represent a large family including the biharmonic surfaces) in the Bianchi-Cartan-Vranceanu spaces, obtaining the characterization of those with constant angle and of those which are $\mathrm{SO}(2)$-invariant. Furthermore, we characterize the constant angle surfaces of the three-dimensional Euclidean space which have bi-harmonic Gauss map, proving that they are Hopf cylinders over a clothoid.

Also, we characterize the constant angle surfaces of $\mathrm{SL}(2, \mathbb{R})$. In particular, we give an explicit local description of these surfaces by means of a suitable curve of $\operatorname{SL}(2, \mathbb{R})$ and a 1-parameter family of isometries of $\operatorname{SL}(2, \mathbb{R})$. 
Keywords: biharmonic immersions, biconservative immersions, surfaces with biharmonic Gauss map, constant angle surfaces. 
$\begin{array}{lll}1 & \text { Curvas bi-harmônicas no grupo especial linear } & 7\end{array}$

1.1 o conceito de biharmonicidade . . . . . . . . . . . . . . . . 7

1.1 .1 Curvas bi-harmônicas . . . . . . . . . . . . . . . 9

1.2 o grupo especial linear $\ldots \ldots \ldots \ldots \ldots \ldots \ldots \ldots \ldots$

1.3 Curvas bi-harmônicas em $\mathrm{SL}(2, \mathbb{R})_{-} \ldots \ldots \ldots \ldots \ldots \ldots$

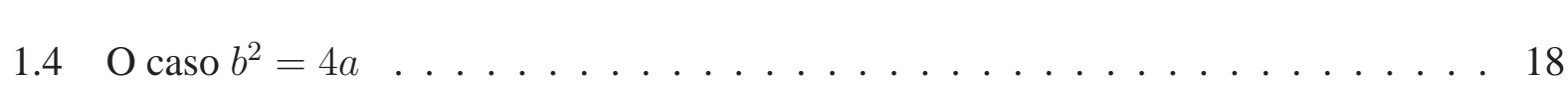

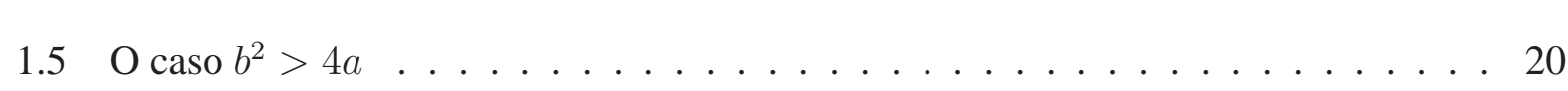

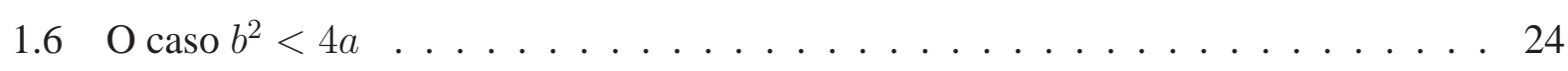

2 Superfícies biconservativas em BCV-espaços

2.1 Espaços de Bianchi-Cartan-Vranceanu e sua estrutura Riemanniana . . . . . . . . 29 
2.2 Equações de compatibilidade . . . . . . . . . . . . . . . . . . 32

2.3 o problema de biconservatividade . . . . . . . . . . . . . . . . 39

2.4 Superfícies biconservativas com ângulo constante em BCV-espaços . . . . . . . . 42

2.5 Superfícies de revolução biconservativas . . . . . . . . . . . . . . . . . . . 47

2.5.1 Superfícies de revolução biconservativas em BCV-espaços . . . . . . . . . 49

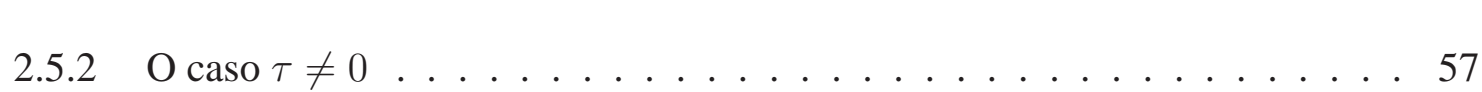

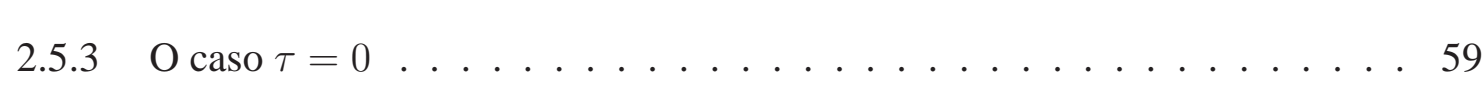

3 Superfícies com aplicação de Gauss bi-harmônicas $\quad 63$

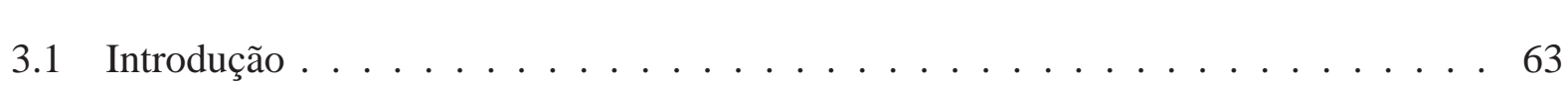

3.2 Superfícies de ângulo constante com aplicação de Gauss bi-harmônical . . . . . . . 65

4 Superfícies de ângulo constante no grupo especial linear $\quad 71$

4.1 Superfícies de ângulo constante em $\mathrm{SL}(2, \mathbb{R})_{\pi} \ldots \ldots \ldots$. . . . . . . . . 77

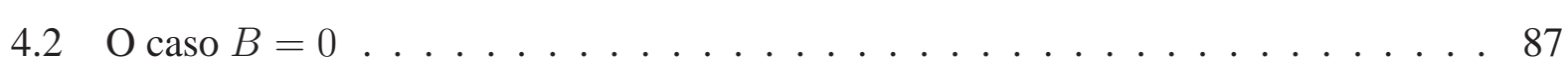

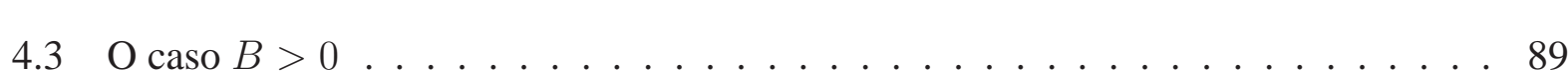

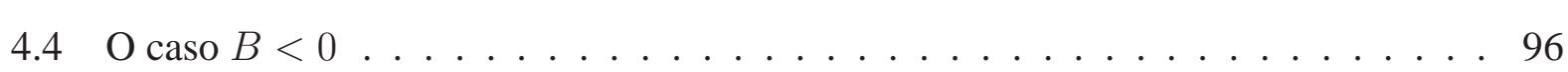

$\begin{array}{ll}\text { Referências Bibliográficas } & 103\end{array}$ 
Este trabalho é dedicado ao estudo de problemas relacionados com a teoria de bi-harmonicidade (Capítulos 1, 2] 3) e de superfícies de ângulos constante (Capítulo 4).

Um tema de grande relevância na geometria diferencial é constituído pela teoria de harmonicidade. Em particular, o estudo das imersões isométricas harmônicas se destaca devido ao fato de ser equivalente ao estudo das imersões mínimas (ver [29]). Lembramos que uma aplicação é dita harmônica se é ponto crítico do funcional energia (ver Definição 1.1). Uma generalização destas aplicações, sugerida por Eells-Lamaire em [28], é obtida ao considerarmos os pontos críticos do chamado funcional bienergia, que será definido na Seção 1.1. As aplicações que satisfazem esta condição são conhecidas como aplicações bi-harmônicas.

O estudo das funções bi-harmônicas teve início em 1863 devido a sua ligação com a teoria da elasticidade e com a mecânica dos fluidos; G.B. Airy e J.C. Maxwell foram os primeiros a usar tal tipo de funções para descrever um modelo matemático de elasticidade (veja [1,47]). Já a teoria das funções poli-harmônicas se desenvolveu mais tarde por obra de E. Almansi, T. Levi-Civita e M. Nicolaescu. O estudo das funções harmônicas e poli-harmônicas em variedades Riemannianas 
teve início na década de 70 com o trabalho de L. Sario, M. Nakai, C. Wang e L. Chung (veja [22]), seguido pelo trabalho de R. Caddeo e L. Vanhecke publicado em 1986 (ver [14]).

Nos últimos quinze anos, o estudo das aplicações e das imersões bi-harmônicas tem gerado nos pesquisadores um interesse crescente como demonstra a lista das publicações sobre o assunto presente na bibliografia do artigo [49].

No Capítulo 1 analisamos o conceito de bi-ionicidade para curvas e as estudamos no grupo especial linear $\operatorname{SL}(2, \mathbb{R})$, munido de uma família de métricas $g_{\tau}$ que depende de um parâmetro $\tau$. Os resultados deste estudo foram estão presentes no artigo [56]. Primeiramente demonstramos que as curvas bi-harmônicas de $\left(\mathrm{SL}(2, \mathbb{R}), g_{\tau}\right)$ fazem um ângulo constante $\vartheta$ com o campo de vetores tangente à fibração de Hopf (ver Seção 1.2). Logo após, provamos que a equação diferencial

$$
\gamma^{I V}+\left(b^{2}-2 a\right) \gamma^{\prime \prime}+a^{2} \gamma=0
$$

onde $a$ e $b$ são constantes que dependem de $\vartheta$ e $\tau$, deve ser satisfeita por uma curva bi-harmônica própria em $\operatorname{SL}(2, \mathbb{R})$, vista como curva do espaço pseudo-Euclidiano $\mathbb{R}_{2}^{4}$. Separamos o estudo em três casos que dependem do sinal da constante $\left(b^{2}-4 a\right)$ obtendo, em cada um deles, as expressões destas curvas como curvas em $\mathbb{R}_{2}^{4}$.

O Capítulo 2 é dedicado ao estudo das superfícies biconservativas nos espaços de BianchiCartan-Vranceanu (BCV-espaços). Para este fim, começamos apresentando o conceito de biconservatividade e mostrando sua relação com o de bi-harmonicidade. Em seguida, foram descritos os BCV-espaços e sua estrutura Riemanniana, os quais constituem uma representação local para as variedades Riemannianas homogêneas com grupo de isometria de dimensão 4 e para aquelas com curvatura seccional constante não negativa, dada pela seguinte família a dois parâmetros de métricas Riemannianas:

$$
g_{\kappa, \tau}=\frac{d x^{2}+d y^{2}}{F^{2}}+\left(d z+\tau \frac{y d x-x d y}{F}\right)^{2}, \quad \kappa, \tau \in \mathbb{R},
$$

onde $F=1+\frac{\kappa}{4}\left(x^{2}+y^{2}\right)$, definidas em

$$
\mathcal{N}=\left\{(x, y, z) \in \mathbb{R}^{3}: 1+\frac{\kappa}{4}\left(x^{2}+y^{2}\right)>0\right\} .
$$


Fazendo uso da fibração de Hopf admitida por estes espaços, utilizamos as técnicas desenvolvida em [18] para fazer o estudo das superfícies biconservativas de ângulo constante, obtendo no Teorema 2.13 a caracterização destas. Posteriormente, consideramos as superfícies de revolução biconservativas nos BCV-espaços. Dividimos o estudo destas superfícies nos casos $\tau \neq 0$ e $\tau=0$. Para o caso $\tau \neq 0$, no Teorema 2.18, concluímos que tais superfícies devem ser cilindros de Hopf. Já no caso $\tau=0$ obtemos no Teorema 2.19 um sistema de equações diferenciais que caracteriza estas superfícies, embora não tenhamos obtido uma forma de integrar tal sistema.

Um resultado clássico da teoria das aplicações harmônicas, conhecido como Teorema de RuhVilms (ver [64]) afirma que a aplicação de Gauss associada a uma imersão isométrica

$$
\gamma: \mathcal{M}^{m} \subset \mathbb{R}^{m+n} \rightarrow G(m, n)
$$

que a cada ponto $p$ de $\mathcal{M}$ associa o espaço tangente $T_{p} \mathcal{M}$, visto como um ponto da variedade Grassmanniana dos subespaços $m$-dimensionais orientados de $\mathbb{R}^{m+n}$, é harmônica se, e somente se, o campo curvatura média de $\mathcal{M}$ é paralelo. Em [6], A. Balmuş, S. Montaldo e C. Oniciuc, sugerem uma generalização do Teorema de Ruh-Vilms, considerando o problema de caracterizar as superfícies que possuem aplicação de Gauss bi-harmônica e obtêm uma condição que deve ser satisfeita por estas (ver Teorema 3.1). Seguindo nesta direção, no Capítulo 3, estudamos as superfícies do espaço Euclidiano tridimensional de ângulo constante que possuem aplicação de Gauss bi-harmônica própria. Como resultado, obtemos que as únicas superfícies que satisfazem estas hipóteses são cilindros sobre uma clotóide, também conhecida como espiral de Cornu.

Outro assunto relacionado com a teoria de harmonicidade que tem despertado grande interesse de pesquisadores renomados da área de geometria diferencial é o estudo de superfícies cujo campo de vetores normal unitário faz um ângulo constante com uma direção fixa do espaço ambiente (ver [20, 21, 24, 25, 33, 43, 51, 65], entre outros). Este interesse foi motivado pelo trabalho [16] de Cermelli e Di Scala que analisaram o caso de superfícies de ângulo constante em $\mathbb{R}^{3}$ obtendo uma importante relação com a equação de Hamilton-Jacobi e mostrando sua aplicação no campo da física, na configuração de equilíbrio de cristais líquidos. A relação entre tais superfícies e 
os cristais líquidos reside no fato das moléculas destes, em sua fase nemática (ver Capítulo 4), apresentarem (naturalmente) uma tendência em se alinharem de acordo com uma direção dada por um campo $X$, que é um ponto crítico do funcional energia

$$
E(X)=\frac{1}{2} \int_{\Omega}\|d X\|^{2} d v
$$

onde $\Omega$ é um domínio de $\mathbb{R}^{3}$ sobre o qual o campo $X$ é definido de acordo com a disposição do cristal líquido. A equação de Euler para este caso, descrita por Eells e Sampson em [29], é

$$
\Delta X+\|d X\|^{2} X=0
$$

Estes campos são conhecidos como campos harmônicos. A fronteira que separa os cristais líquidos de sua fase nemática e sua fase isotrópica (ver Capítulo 4) pode ser vista como uma superfície que faz ângulo constante com o campo $X$. Naturalmente o primeiro exemplo de campo harmônico a ser considerado é aquele constante, o que dá origem ao estudo das superfícies de ângulo constante.

As superfícies de ângulo constante foram, então, consideradas em diversas geometrias tridimensionais. Uma atenção especial foi dada as 3-variedades Riemannianas homogêneas com grupo de isometria de dimensão 4 . Os espaços produtos $\mathbb{S}^{2} \times \mathbb{R}$ e $\mathbb{H}^{2} \times \mathbb{R}$ foram considerados por Dillen-Fastenakels-Van der Veken-Vrancken (ver [21]), e Dillen-Munteanu (ver [20]), respectivamente. Em seguida, no artigo [33], Fastenakels-Munteanu-Van der Veken estenderam a noção de superfícies de ângulo constante para os BCV-espaços e deram sua classificação para o grupo de Heisenberg. Além disso, Montaldo-Onnis, em [51], caracterizaram as superfícies do tipo hélice na família a um parâmetro das esferas de Berger $\mathbb{S}_{\epsilon}^{3}$, com $\epsilon>0$.

O espaço tridimensional homogêneo com grupo de isometria de dimensão 4 remanescente, até então, era o grupo especial linear. Desta forma, no Capítulo 4 consideramos as superfícies cujo campo de vetores normal unitário $N$ faz ângulo constante $\vartheta$ com o campo de Hopf de $\left(\mathrm{SL}(2, \mathbb{R}), g_{\tau}\right)$ (como na Seção 1.2). Dividimos, então, o nosso estudo em três casos, dependendo da constante

$$
B:=\left(\tau^{2}+1\right) \cos ^{2} \vartheta-1
$$


a saber: $B>0, B=0, B<0$. Para cada um desses casos obtemos uma equação diferencial que deve ser satisfeita pelo vetor posição dessas superfícies vistas como superfícies de $\mathbb{R}_{2}^{4}$ (ver Proposição 4.5). Enfim, classificamos estas superfícies através dos Teoremas 4.7, 4.9 e 4.11, nos quais demonstramos que, em cada um dos três casos, estas podem ser obtidas pela ação de um subgrupo do grupo de isometrias de $\left(\operatorname{SL}(2, \mathbb{R}), g_{\tau}\right)$ sobre uma curva de $\operatorname{SL}(2, \mathbb{R})$. Ressaltamos que os resultados presentes neste capítulo foram publicados no artigo [52]. 



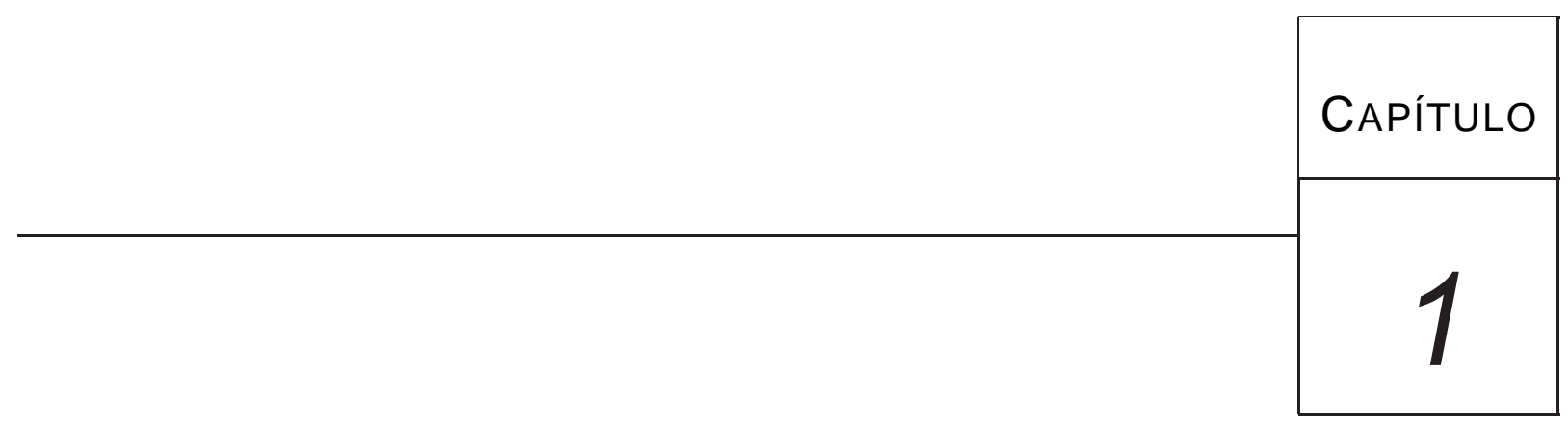

\section{Curvas bi-harmônicas no grupo} especial linear

\subsection{O conceito de biharmonicidade}

Indicaremos por $C^{\infty}(\mathcal{M}, \mathcal{N})$ o espaço das aplicações diferenciáveis $\phi:(\mathcal{M}, g) \rightarrow(\mathcal{N}, h)$ entre as duas variedades Riemannianas $(\mathcal{M}, g)$ e $(\mathcal{N}, h)$.

Definição 1.1. Uma aplicação $\phi \in C^{\infty}(\mathcal{M}, \mathcal{N})$ é dita harmônica se é um ponto crítico do funcional energia:

$$
E: C^{\infty}(\mathcal{M}, \mathcal{N}) \rightarrow \mathbb{R}, \quad E(\phi)=\frac{1}{2} \int_{\mathcal{M}}\|\mathrm{d} \phi\|^{2} \mathrm{~d} \mathcal{M}
$$


ou seja, se ф é solução da correspondente equação de Euler-Lagrange dada por

$$
\tau(\phi):=\operatorname{tr} \widetilde{\nabla} \mathrm{d} \phi=0
$$

onde

$$
\left(\widetilde{\nabla}_{X} \mathrm{~d} \phi\right)(Y)=\bar{\nabla}_{\mathrm{d} \phi(X)} \mathrm{d} \phi(Y)-\mathrm{d} \phi\left(\nabla_{X} Y\right), \quad X, Y \in T \mathcal{M},
$$

sendo $\bar{\nabla}$ a conexão de $\mathcal{N}$ e $\nabla$ a de $\mathcal{M}$. O campo $\tau(\phi)$ é conhecido como campo de tensão de $\phi$ (ver [29]).

Paralelamente à Definição 1.1 indicando $\operatorname{por} \operatorname{Imm}(\mathcal{M}, \mathcal{N})$ o espaço das imersões Riemannianas em $(\mathcal{N}, h)$, temos a seguinte

Definição 1.2. Uma imersão Riemanniana $\phi:\left(\mathcal{M}, \phi^{*} h\right) \rightarrow(\mathcal{N}, h)$ é dita mínima se é um ponto crítico do funcional volume

$$
V: \operatorname{Imm}(\mathcal{M}, \mathcal{N}) \rightarrow \mathbb{R}, \quad V(\phi)=\frac{1}{2} \int_{\mathcal{M}} v_{\phi^{*} h}
$$

Neste caso a correspondente equação de Euler-Lagrange é $H=0$, onde $H$ é o campo de vetores curvatura média.

Em [29], J. Eells e J.H. Sampson provaram que uma imersão Riemanniana $\phi:(\mathcal{M}, g) \rightarrow$ $(\mathcal{N}, h)$ é mínima se, e somente, se é um ponto crítico do funcional energia. Logo, estudar as imersões Riemannianas mínimas é equivalente a estudar as imersões Riemannianas harmônicas.

Uma extensão natural do conceito de aplicação harmônica, e portanto de imersão mínima, é obtida considerando o funcional bienergia:

$$
E_{2}: C^{\infty}(\mathcal{M}, \mathcal{N}) \rightarrow \mathbb{R}, \quad E_{2}(\phi)=\frac{1}{2} \int_{\mathcal{M}}\|\tau(\phi)\|^{2} \mathrm{~d} \mathcal{M}
$$

Mais precisamente,

Definição 1.3. Uma aplicação $\phi \in C^{\infty}(\mathcal{M}, \mathcal{N})$ é dita bi-harmônica se é um ponto crítico do funcional bienergia. 
Em [39,40] G.Y. Jiang obteve a fórmula da primeira variação da bi-energia, provando que a equação de Euler-Lagrange para $E_{2}$ é dada por

$$
\tau_{2}(\phi):=-\Delta^{\phi} \tau(\phi)+\operatorname{tr} \mathrm{R}^{\mathcal{N}}(d \phi, \tau(\phi)) d \phi=0
$$

onde $\mathrm{R}^{\mathcal{N}}$ denota o tensor de curvatura da variedade $\mathcal{N}$, dado por

$$
\mathrm{R}^{\mathcal{N}}(X, Y) Z=\bar{\nabla}_{Y} \bar{\nabla}_{X} Z-\bar{\nabla}_{X} \bar{\nabla}_{Y} Z+\bar{\nabla}_{[X, Y]} Z
$$

para $X, Y, Z \in T \mathcal{N}$, enquanto $\Delta^{\phi}$ denota o Laplaciano generalizado agindo em $C\left(\phi^{-1} T \mathcal{N}\right)$, que com respeito a um referencial local ortonormal $\left\{E_{i}\right\}_{i=1}^{m}$ de $\mathcal{M}$ é dado por

$$
\Delta^{\phi}=-\operatorname{tr}\left(\nabla^{\phi}\right)^{2}=-\sum_{i=1}^{m}\left\{\nabla_{E_{i}}^{\phi} \nabla_{E_{i}}^{\phi}-\nabla_{\nabla_{E_{i}} E_{i}}^{\phi}\right\} \text {, }
$$

sendo $\nabla^{\phi}$ a conexão em $C\left(\phi^{-1} T \mathcal{N}\right)$ induzida pela conexão de Levi-Civita de $(\mathcal{N}, h)$. O campo $\tau_{2}(\phi)$ definido acima é chamado de campo de bitensão de $\phi$, enquanto a equação (1.1) é conhecida como equação bi-harmônica.

Da expressão do campo de bitensão $\tau_{2}$ segue que toda aplicação harmônica (i.e. $\tau=0$ ) é também bi-harmônica. Uma aplicação bi-harmônica que não é harmônica é dita aplicação biharmônica própria.

\subsubsection{Curvas bi-harmônicas}

Seja $(\mathcal{N}, h)$ uma variedade Riemanniana. Uma curva $\gamma: I \rightarrow(\mathcal{N}, h)$, parametrizada por comprimento de arco, é bi-harmônica se $\gamma$ for uma aplicação bi-harmônica, ou seja, se

$$
\bar{\nabla}_{\gamma^{\prime}}^{3} \gamma^{\prime}+\mathrm{R}\left(\gamma^{\prime}, \bar{\nabla}_{\gamma^{\prime}} \gamma^{\prime}\right) \gamma^{\prime}=0
$$

Chamamos de curvas bi-harmônicas próprias aquelas definidas a partir de aplicações bi-harmônicas próprias. 
O estudo das curvas bi-harmônicas próprias em superfícies curvas começou com R. CaddeoS. Montaldo-P. Piu. Biharmonic em [12], onde são descritas estas curvas em superfícies, provando que curvas bi-harmônicas em superfícies de curvatura Gaussiana não positiva são geodésicas.

Para variedades Riemannianas tridimensionais com curvatura seccional constante, os casos de curvatura nula e negativa são considerados em [26] e [9], onde mostra-se que as únicas curvas bi-harmônicas são as geodésicas. Além disso, em [8], é considerado o caso de curvatura positiva, mostrando que as curvas bi-harmônicas possuem curvatura geodésica e torção geodésica constantes.

Feita exceção às formas espaciais, os espaços Riemannianos tridimensionais homogêneos mais interessantes são aqueles que possuem grupo de isometrias com dimensão 4: as esferas de Berger, o grupo de Heisenberg, o grupo especial linear $\operatorname{SL}(2, \mathbb{R})$, e os produtos Riemannianos $\mathbb{S}^{2} \times \mathbb{R}$ e $\mathbb{H}^{2} \times \mathbb{R}$, onde $\mathbb{S}^{2}$ e $\mathbb{H}^{2}$ são a esfera e o plano hiperbólico bidimensionais, respectivamente.

Em [4] A. Balmuş determinou as equações que caracterizam as curvas bi-harmônicas para as esferas de Berger $\mathbb{S}_{\epsilon}^{3}$ como curvas em $\mathbb{R}^{4}$ e deu uma interpretação geométrica para as curvas biharmônicas na esfera Euclidiana unitária $\mathbb{S}^{3}$. Em [13] os autores provaram que qualquer curva bi-harmônica no grupo de Heisenberg é uma hélice e deram suas parametrizações explícitas.

Além disso, em [11] os autores consideraram as curvas bi-harmônicas próprias nos espaços de Bianchi-Cartan-Vranceanu $\widetilde{\mathrm{SL}}(2, \mathbb{R}), S U(2), \mathbb{S}^{2} \times \mathbb{R}$ e $\mathbb{H}^{2} \times \mathbb{R}$ (os quais serão descritos na Seção 2.1), provando que essas curvas são hélices e dando suas equações paramétricas.

Neste capítulo apresentamos os resultados que compõem o artigo [56], onde é feito o estudo das curvas bi-harmônicas próprias no grupo especial linear $\mathrm{SL}(2, \mathbb{R})$ munido de uma família a um-parâmetro de métricas como descrito na Seção 1.2. Usando as mesmas técnicas apresentadas em [4] (para o caso das esferas de Berger) e em [13] (para o caso do grupo de Heisenberg), concluímos que as curvas bi-harmônicas de $\mathrm{SL}(2, \mathbb{R})$ fazem um ângulo constante $\vartheta$ com o campo de 
vetores tangente à fibração de Hopf. Ainda, no Teorema 1.7 provamos que a equação diferencial

$$
\gamma^{I V}+\left(b^{2}-2 a\right) \gamma^{\prime \prime}+a^{2} \gamma=0
$$

onde $a$ e $b$ são constantes que dependem de $\vartheta$ e $\tau$, deve ser satisfeita por uma curva bi-harmônica própria em $\operatorname{SL}(2, \mathbb{R})$, vista como curva do espaço pseudo-Euclidiano $\mathbb{R}_{2}^{4}$. Separamos o estudo em três casos que dependem do sinal da constante $\left(b^{2}-4 a\right)$ obtendo, em cada caso, as expressões destas curvas como curvas em $\mathbb{R}_{2}^{4}$.

\subsection{O grupo especial linear}

Seja $\mathbb{R}_{2}^{4}$ o espaço pseudo-Euclidiano 4-dimensional equipado com o produto interno semidefinido de assinatura $(2,2)$ dado por

$$
\langle v, w\rangle=v_{1} w_{1}+v_{2} w_{2}-v_{3} w_{3}-v_{4} w_{4}, \quad v, w \in \mathbb{R}^{4}
$$

Identificaremos o grupo especial linear com

$$
\operatorname{SL}(2, \mathbb{R})=\left\{(z, w) \in \mathbb{C}^{2}:|z|^{2}-|w|^{2}=1\right\}=\left\{v \in \mathbb{R}_{2}^{4}:\langle v, v\rangle=1\right\} \subset \mathbb{R}_{2}^{4} .
$$

Tal identificação pode ser obtida através da transformação linear

$$
\left(\begin{array}{ll}
a & b \\
c & d
\end{array}\right) \longrightarrow \frac{1}{2}((a+d)+i(b-c),(b+c)+i(a-d)),
$$

com $a d-b c=1$, a qual é um difeomorfismo. Além disso, usaremos o modelo de Lorentz para o plano hiperbólico com curvatura Gaussiana constante -4 , como segue

$$
\mathbb{H}^{2}(-4)=\left\{(x, y, z) \in \mathbb{R}_{1}^{3}: x^{2}+y^{2}-z^{2}=-1 / 4\right\}
$$

onde $\mathbb{R}_{1}^{3}$ é o espaço de Minkowski tridimensional. A aplicação de $\operatorname{Hopf} \psi: \operatorname{SL}(2, \mathbb{R}) \rightarrow \mathbb{H}^{2}(-4)$ dada por

$$
\psi(z, w)=\frac{1}{2}\left(2 z \bar{w},|z|^{2}+|w|^{2}\right)
$$


é uma submersão, com fibras circulares. Tomando-se

$$
X_{1}(z, w)=(i z, i w), \quad X_{2}(z, w)=(i \bar{w}, i \bar{z}), \quad X_{3}(z, w)=(\bar{w}, \bar{z}), \quad(z, w) \in \operatorname{SL}(2, \mathbb{R}),
$$

resulta que $X_{1}$ é um campo de vetores vertical (i.e. $\mathrm{d} \psi\left(X_{1}\right)=0$ ), enquanto que $X_{2}, X_{3}$ são horizontais. O campo de vetores $X_{1}$ é chamado de campo de Hopf.

Equiparemos $\mathrm{SL}(2, \mathbb{R})$ com a família a um parâmetro de métricas $g_{\tau}, \tau>0$, dada por

$$
g_{\tau}\left(X_{i}, X_{j}\right)=\delta_{i j}, \quad g_{\tau}\left(X_{1}, X_{1}\right)=\tau^{2}, \quad g_{\tau}\left(X_{1}, X_{j}\right)=0, \quad i, j \in\{2,3\}
$$

a qual torna a aplicação de Hopf $\psi:\left(\operatorname{SL}(2, \mathbb{R}), g_{\tau}\right) \rightarrow \mathbb{H}^{2}(-4)$ uma submersão Riemanniana. Com respeito ao produto interno de $\mathbb{R}_{2}^{4}$ a métrica $g_{\tau}$ é dada por

$$
g_{\tau}(X, Y)=-\langle X, Y\rangle+\left(1+\tau^{2}\right)\left\langle X, X_{1}\right\rangle\left\langle Y, X_{1}\right\rangle .
$$

De agora em diante, denotaremos a 3-variedade homogênea $\left(\mathrm{SL}(2, \mathbb{R}), g_{\tau}\right)$ por $\mathrm{SL}(2, \mathbb{R})_{\tau}$. Temos que

$$
E_{1}=-\tau^{-1} X_{1}, \quad E_{2}=X_{2}, \quad E_{3}=X_{3},
$$

é uma base ortonormal em $\operatorname{SL}(2, \mathbb{R})_{\tau}$ e a conexão de Levi-Civita $\nabla^{\tau}$ de $\operatorname{SL}(2, \mathbb{R})_{\tau}$ é dada por:

$$
\begin{aligned}
& \nabla_{E_{1}}^{\tau} E_{1}=0, \quad \nabla^{\tau} E_{2} E_{2}=0, \quad \nabla^{\tau} E_{3} E_{3}=0, \\
& \nabla^{\tau}{ }_{E_{1}} E_{2}=-\tau^{-1}\left(2+\tau^{2}\right) E_{3}, \quad \nabla^{\tau}{ }_{E_{1}} E_{3}=\tau^{-1}\left(2+\tau^{2}\right) E_{2}, \\
& \nabla^{\tau}{ }_{E_{2}} E_{1}=-\tau E_{3}, \quad \nabla^{\tau}{ }_{E_{3}} E_{1}=\tau E_{2}, \quad \nabla^{\tau} E_{3} E_{2}=-\tau E_{1}=-\nabla^{\tau} E_{2} E_{3} .
\end{aligned}
$$

Além disso, as componentes não nulas da curvatura Riemanniana são

$$
\begin{aligned}
& g_{\tau}\left(\mathrm{R}\left(E_{1}, E_{2}\right) E_{1}, E_{2}\right)=\tau^{2}, \quad g_{\tau}\left(\mathrm{R}\left(E_{1}, E_{3}\right) E_{1}, E_{3}\right)=\tau^{2}, \\
& g_{\tau}\left(\mathrm{R}\left(E_{2}, E_{3}\right) E_{2}, E_{3}\right)=-\left(4+3 \tau^{2}\right) .
\end{aligned}
$$

Finalmente, lembramos que o grupo de isometria de $\operatorname{SL}(2, \mathbb{R})_{\tau}$ é o grupo unitário indefinido 4-dimensional $\mathrm{U}_{1}(2)$ que pode ser identificado com:

$$
\mathrm{U}_{1}(2)=\left\{A \in \mathrm{O}_{2}(4): A J_{1}= \pm J_{1} A\right\},
$$


onde $J_{1}$ é a estrutura complexa de $\mathbb{R}^{4}$ definida por

$$
J_{1}=\left(\begin{array}{ll}
J & 0 \\
0 & J
\end{array}\right), \quad J=\left(\begin{array}{cc}
0 & -1 \\
1 & 0
\end{array}\right)
$$

enquanto

$$
\mathrm{O}_{2}(4)=\left\{A \in \mathrm{GL}(4, \mathbb{R}): A^{t}=\epsilon A^{-1} \epsilon\right\}, \quad \epsilon=\left(\begin{array}{cc}
I & 0 \\
0 & -I
\end{array}\right), \quad I=\left(\begin{array}{ll}
1 & 0 \\
0 & 1
\end{array}\right)
$$

é o grupo ortogonal indefinido. Observamos que $\mathrm{O}_{2}(4)$ é o grupo das matrizes reais $4 \times 4$ que preservam o produto interno semi-definido de $\mathbb{R}_{2}^{4}$.

\subsection{Curvas bi-harmônicas em $\mathrm{SL}(2, \mathbb{R})_{\tau}$}

Seja $\gamma: I \rightarrow \operatorname{SL}(2, \mathbb{R})_{\tau}$ uma curva diferenciável parametrizada por comprimento de arco e seja $\{T, N, B\}$ o triedro de campos de vetores ortonormais tangentes a $\operatorname{SL}(2, \mathbb{R})_{\tau}$ ao longo de $\gamma(s)$ definido como segue: denotamos por $T$ o campo de vetores unitário $\gamma^{\prime}$ tangente a $\gamma$, por $N$ o campo de vetores unitário na direção de $\nabla^{\tau} T$ normal a $\gamma$, e escolhemos $B$ de forma que $\{T, N, B\}$ seja uma base ortonormal positivamente orientada. Então, temos as seguintes equações de Frenet

$$
\left\{\begin{array}{l}
\nabla_{T}^{\tau} T=k_{1} N, \\
\nabla_{T}^{\tau} N=-k_{1} T+k_{2} B, \\
\nabla_{T}^{\tau_{T} B}=-k_{2} N,
\end{array}\right.
$$

onde $k_{1}=\left\|\nabla_{T}^{\tau} T\right\|$ é a curvatura geodésica de $\gamma$ e $k_{2}$ sua torção.

Teorema 1.4. Considere $\gamma: I \rightarrow \operatorname{SL}(2, \mathbb{R})_{\tau}$ uma curva ppca e a notação $T=\sum T_{i} E_{i}, N=$ $\sum N_{i} E_{i}$ e $B=\sum B_{i} E_{i}$. Então $\gamma$ é bi-harmônica própria se, e somente se,

$$
\left\{\begin{array}{l}
k_{1}=\text { constante } \neq 0, \\
k_{1}^{2}+k_{2}^{2}=\tau^{2}-4\left(1+\tau^{2}\right) B_{1}^{2}, \\
k_{2}^{\prime}=-4\left(1+\tau^{2}\right) N_{1} B_{1} .
\end{array}\right.
$$


Demonstração. Considere a curva $\gamma: I \rightarrow \operatorname{SL}(2, \mathbb{R})_{\tau}$ parametrizada por comprimento de arco. Neste caso a equação (1.2) se torna

$$
\left(\nabla^{\tau} T\right)^{3} T+\mathrm{R}\left(T, \nabla_{T}^{\tau} T\right) T=0
$$

Usando as equações de Frenet em (1.8), obtemos as condições

$$
\left\{\begin{array}{l}
k_{1}=\text { constante } \neq 0, \\
k_{1}^{2}+k_{2}^{2}=g_{\tau}(\mathrm{R}(T, N) T, N), \\
k_{2}^{\prime}=-g_{\tau}(\mathrm{R}(T, N) T, B) .
\end{array}\right.
$$

Escrevendo

$$
T=\sum_{i=1}^{3} T_{i} E_{i}, \quad N=\sum_{i=1}^{3} N_{i} E_{i}, \quad B=\sum_{i=1}^{3} B_{i} E_{i}
$$

e usando (1.5), temos que

$$
\begin{aligned}
& g_{\tau}(\mathrm{R}(T, N) T, N)=\tau^{2}-4\left(1+\tau^{2}\right) B_{1}^{2}, \\
& g_{\tau}(\mathrm{R}(T, N) T, B)=4\left(1+\tau^{2}\right) N_{1} B_{1} .
\end{aligned}
$$

Proposição 1.5. Seja $\gamma: I \rightarrow \mathrm{SL}(2, \mathbb{R})_{\tau}$ uma curva bi-harmônica própria parametrizada pelo comprimento de arco, então sua curvatura geodésica e sua torção são constantes.

Demonstração. Das equações de Frenet resulta que

$$
g_{\tau}\left(\nabla_{T}^{\tau} B, E_{1}\right)=-g_{\tau}\left(k_{2} N, E_{1}\right)=-k_{2} N_{1} .
$$

Por outro lado, usando (1.4), obtemos

$$
\begin{aligned}
g_{\tau}\left(\nabla_{T}^{\tau} B, E_{1}\right) & =g_{\tau}\left(B_{1}^{\prime} E_{1}+T_{2} B_{3} \nabla_{E_{2}}^{\tau} E_{3}+T_{3} B_{2} \nabla_{E_{3}}^{\tau} E_{2}, E_{1}\right) \\
& =B_{1}^{\prime}+\tau\left(T_{2} B_{3}-T_{3} B_{2}\right) \\
& =B_{1}^{\prime}-\tau N_{1} .
\end{aligned}
$$


Combinando essas duas equações, temos

$$
B_{1}^{\prime}=\left(\tau-k_{2}\right) N_{1}
$$

Agora, usando a segunda equação de (1.7) obtemos

$$
k_{2} k_{2}^{\prime}=-4\left(1+\tau^{2}\right) B_{1} B_{1}^{\prime} .
$$

Da terceira equação de (1.7), (1.9) e (1.10) resulta que $\left(\tau-2 k_{2}\right) B_{1} N_{1}=0$. Portanto, temos duas possibilidades: $B_{1} N_{1}=0$ o que, junto com (1.7), implica que $k_{2}^{\prime}=0$; ou $k_{2}=\tau / 2$. Logo, $k_{2}$ é constante.

Proposição 1.6. Seja $\gamma: I \rightarrow \mathrm{SL}(2, \mathbb{R})_{\tau}$ uma curva bi-harmônica própria parametrizada pelo comprimento de arco, então ela forma um ângulo constante $\vartheta \in(0, \pi / 2]$ com o campo de Hopf $E_{1}$ e seu campo de vetores tangente pode ser escrito como

$$
\gamma^{\prime}(s)=T=\cos \vartheta E_{1}+\operatorname{sen} \vartheta \operatorname{sen} \beta(s) E_{2}+\operatorname{sen} \vartheta \cos \beta(s) E_{3},
$$

onde $\beta: I \rightarrow \mathbb{R}$ é uma função suave.

Demonstração. Primeiro notamos que $B_{1} \neq 0$. De fato se $B_{1}=0$ e $N_{1}=0$, então $\gamma$ é a curva integral do campo de vetores $E_{1}$ e logo é uma geodésica. Além disso, se $B_{1}=0$ e $N_{1} \neq 0$, de (1.9) obtemos que $k_{2}=\tau$ o que, junto com a segunda equação de (1.7), nos dá $k_{1}=0$.

Como $B_{1} \neq 0$, a terceira equação de (1.7) e a Proposição 1.5 implicam que $N_{1}=0$. Agora, usando as equações (1.4) e (1.6) temos que

$$
k_{1} N_{1}=g_{\tau}\left(\nabla_{T}^{\tau} T, E_{1}\right)=T_{1}^{\prime}
$$

Concluímos que $T_{1}=$ constante e obtemos a expressão (1.11).

Usando o resultado anterior temos o seguinte 
Teorema 1.7. Seja $\gamma: I \rightarrow \operatorname{SL}(2, \mathbb{R})_{\tau} \subset \mathbb{R}_{2}^{4}$ uma curva parametrizada por comprimento de arco. Então $\gamma$ é bi-harmônica própria se, e somente se, como curva em $\mathbb{R}_{2}^{4}$, satisfaz a equação

$$
\gamma^{I V}+\left(b^{2}-2 a\right) \gamma^{\prime \prime}+a^{2} \gamma=0
$$

onde a e b são as constantes dadas por:

$$
\left\{\begin{array}{l}
a=\frac{1}{2}\left(-\tau^{-2}+1-\left(1+\tau^{-2}\right) \cos 2 \vartheta\right)-\tau^{-1} \cos \vartheta \beta^{\prime} \\
b=\beta^{\prime}=-\tau^{-1}\left(2+\tau^{2}\right) \cos \vartheta \pm \sqrt{\left(4+5 \tau^{2}\right) \cos ^{2} \vartheta-4\left(1+\tau^{2}\right)}
\end{array}\right.
$$

com

$$
\frac{4\left(1+\tau^{2}\right)}{\left(4+5 \tau^{2}\right)} \leq \cos ^{2} \vartheta<1
$$

Demonstração. Escrevendo

$$
\gamma(s)=\left(x_{1}(s), x_{2}(s), x_{3}(s), x_{4}(s)\right)
$$

de (1.11) temos que as funções coordenadas de $\gamma$ em $\mathbb{R}_{2}^{4}$ satisfazem

$$
\left\{\begin{array}{l}
x_{1}^{\prime}=\tau^{-1} \cos \vartheta x_{2}+\operatorname{sen} \vartheta \cos \beta x_{3}+\operatorname{sen} \vartheta \operatorname{sen} \beta x_{4}, \\
x_{2}^{\prime}=-\tau^{-1} \cos \vartheta x_{1}+\operatorname{sen} \vartheta \operatorname{sen} \beta x_{3}-\operatorname{sen} \vartheta \cos \beta x_{4}, \\
x_{3}^{\prime}=\operatorname{sen} \vartheta \cos \beta x_{1}+\operatorname{sen} \vartheta \operatorname{sen} \beta x_{2}+\tau^{-1} \cos \vartheta x_{4}, \\
x_{4}^{\prime}=\operatorname{sen} \vartheta \operatorname{sen} \beta x_{1}-\operatorname{sen} \vartheta \cos \beta x_{2}-\tau^{-1} \cos \vartheta x_{3} .
\end{array}\right.
$$

Derivando 1.13), resulta que

$$
\left\{\begin{array}{l}
x_{1}^{\prime \prime}=a x_{1}-b x_{2}^{\prime} \\
x_{2}^{\prime \prime}=a x_{2}+b x_{1}^{\prime} \\
x_{3}^{\prime \prime}=a x_{3}-b x_{4}^{\prime} \\
x_{4}^{\prime \prime}=a x_{4}+b x_{3}^{\prime}
\end{array}\right.
$$

onde

$$
\left\{\begin{array}{l}
a=\frac{1}{2}\left(-\tau^{-2}+1-\left(1+\tau^{-2}\right) \cos 2 \vartheta\right)-\tau^{-1} \cos \vartheta \beta^{\prime} \\
b=\beta^{\prime}
\end{array}\right.
$$


Agora provaremos que $b$ é constante e determinaremos a sua expressão. Calculando $\nabla^{\tau} T$, usando (1.11) e (1.4), temos que a curvatura geodésica e o campo de vetores normal são dados por

$$
k_{1}= \pm \operatorname{sen} \vartheta\left(\beta^{\prime}+2 \tau^{-1}\left(1+\tau^{2}\right) \cos \vartheta\right), \quad N= \pm\left(\cos \beta E_{2}-\operatorname{sen} \beta E_{3}\right) .
$$

Então

$$
\begin{aligned}
& B=T \wedge N= \pm\left(-\operatorname{sen} \vartheta E_{1}+\cos \vartheta \operatorname{sen} \beta E_{2}+\cos \vartheta \cos \beta E_{3}\right), \\
& k_{2}=g_{\tau}\left(\nabla_{T}^{\tau} N, B\right)=\left(\tau-\cos \vartheta\left(\beta^{\prime}+2 \tau^{-1}\left(1+\tau^{2}\right) \cos \vartheta\right)\right) .
\end{aligned}
$$

Substituindo as expressões de $k_{1}, k_{2}$ e $B_{1}$ na segunda equação de (1.7), resulta que

$$
\beta^{\prime}=-\tau^{-1}\left(2+\tau^{2}\right) \cos \vartheta \pm \sqrt{\left(4+5 \tau^{2}\right) \cos ^{2} \vartheta-4\left(1+\tau^{2}\right)}
$$

Agora, derivando duas vezes (1.14), e usando (1.13), obtemos a equação (1.12). Além disso, como a curva $\gamma$ não é harmônica, de (1.15), resulta que $\cos \vartheta \neq 1$.

Observação 1.1. Para determinar explicitamente a expressão de $\gamma$, nos serão úteis os valores dos produtos internos entre $\gamma$, suas derivadas e a imagem $J_{1}$ sobre estas.

Usando (1.13) e (1.14), obtemos que:

$$
\begin{array}{lll}
\langle\gamma, \gamma\rangle=1, & \left\langle\gamma^{\prime}, \gamma^{\prime}\right\rangle=\tilde{B}, \quad\left\langle\gamma, \gamma^{\prime}\right\rangle=0, \\
\left\langle\gamma^{\prime}, \gamma^{\prime \prime}\right\rangle=0, & \left\langle\gamma^{\prime \prime}, \gamma^{\prime \prime}\right\rangle=D, & \left\langle\gamma, \gamma^{\prime \prime}\right\rangle=-\tilde{B}, \\
\left\langle\gamma^{\prime}, \gamma^{\prime \prime \prime}\right\rangle=-D, & \left\langle\gamma^{\prime \prime}, \gamma^{\prime \prime \prime}\right\rangle=0, & \left\langle\gamma, \gamma^{\prime \prime \prime}\right\rangle=0, \\
\left\langle\gamma^{\prime \prime \prime}, \gamma^{\prime \prime \prime}\right\rangle=E, &
\end{array}
$$

onde

$$
\begin{gathered}
\tilde{B}=\left(1+\tau^{-2}\right) \cos ^{2} \vartheta-1, \quad D=a^{2}+b^{2} \tilde{B}+2 a b \tau^{-1} \cos \vartheta \\
E=a\left(a-2 b^{2}\right) \tilde{B}+b^{2} D-2 a^{2} b \tau^{-1} \cos \vartheta .
\end{gathered}
$$

Além disso, como

$$
J_{1} \gamma=X_{1 \mid \gamma}=-\tau E_{1 \mid \gamma}
$$


usando (1.11) e (1.14), obtemos as seguinte identidades

$$
\begin{aligned}
& \left\langle J_{1} \gamma, \gamma^{\prime}\right\rangle=-\tau^{-1} \cos \vartheta \\
& \left\langle J_{1} \gamma, \gamma^{\prime \prime}\right\rangle=0, \\
& \left\langle J_{1} \gamma^{\prime \prime}, \gamma^{\prime}\right\rangle=-a \tau^{-1} \cos \vartheta-b \tilde{B}:=I, \\
& \left\langle J_{1} \gamma^{\prime}, \gamma^{\prime \prime \prime}\right\rangle=0 \\
& \left\langle J_{1} \gamma^{\prime}, \gamma^{\prime \prime}\right\rangle+\left\langle J_{1} \gamma, \gamma^{\prime \prime \prime}\right\rangle=0, \\
& \left\langle J_{1} \gamma^{\prime \prime}, \gamma^{\prime \prime \prime}\right\rangle+\left\langle J_{1} \gamma^{\prime}, \gamma^{I V}\right\rangle=0 .
\end{aligned}
$$

Para determinar a expressão do vetor posição de $\gamma$ em $\mathbb{R}_{2}^{4}$, integramos (1.12), dividindo nosso estudo em casos, de acordo com as três possibilidades:

(i) $b^{2}=4 a$;

(ii) $b^{2}>4 a$;

(iii) $b^{2}<4 a$.

\subsection{O caso $b^{2}=4 a$}

Teorema 1.8. Seja $\gamma: I \rightarrow \mathrm{SL}(2, \mathbb{R})_{\tau} \subset \mathbb{R}_{2}^{4}$ uma curva bi-harmônica própria parametrizada pelo comprimento de arco tal que $b^{2}=4 a$. Então

$$
b=-\tau^{-1}\left(2+\tau^{2}\right) \cos \vartheta+\sqrt{\left(4+5 \tau^{2}\right) \cos ^{2} \vartheta-4\left(1+\tau^{2}\right)},
$$

com

$$
\cos ^{2} \vartheta=\frac{\left(2+\tau^{2}\right)^{2}}{4+5 \tau^{2}+\tau^{4}}
$$

Além disso,

$$
\begin{aligned}
\gamma(s)= & A\left(\cos (\sqrt{a} s)+g_{14} s \operatorname{sen}(\sqrt{a} s),-\operatorname{sen}(\sqrt{a} s)+g_{14} s \cos (\sqrt{a} s)\right. \\
& \left.-g_{14} s \cos (\sqrt{a} s), g_{14} s \operatorname{sen}(\sqrt{a} s)\right)
\end{aligned}
$$


onde $g_{14}$ é a constante, dada por

$$
g_{14}=\frac{\tau}{\sqrt{4+5 \tau^{2}+\tau^{4}}}
$$

e $A \in \mathrm{O}_{2}(4)$ é uma matriz $4 \times 4$ ortogonal indefinida que comuta com $J_{1}$.

Demonstração. Como $b^{2}=4 a$, a equação diferencial (1.12) se torna

$$
\gamma^{I V}(s)+2 a \gamma^{\prime \prime}(s)+a^{2} \gamma(s)=0
$$

Integrando (1.20) temos

$$
\gamma(s)=\cos (\sqrt{a} s) g_{1}+\operatorname{sen}(\sqrt{a} s) g_{2}+s \cos (\sqrt{a} s) g_{3}+s \operatorname{sen}(\sqrt{a} s) g_{4},
$$

onde $g_{1}, g_{2}, g_{3}$ e $g_{4}$ são vetores constantes de $\mathbb{R}_{2}^{4}$.

Um cálculo direto mostra que $b^{2}=4 a$ ocorre em dois casos: para $\vartheta=0$ e para

$$
\cos ^{2} \vartheta=\frac{\left(2+\tau^{2}\right)^{2}}{4+5 \tau^{2}+\tau^{4}}
$$

e nos dois casos $b$ deve ter a expressão dada em (1.18). Como o primeiro caso produz curvas harmônicas, estudaremos apenas o segundo.

Usando as relações (1.16), obtemos

$$
\begin{aligned}
& \left\langle g_{1}, g_{1}\right\rangle=\left\langle g_{2}, g_{2}\right\rangle=1, \\
& \left\langle g_{3}, g_{3}\right\rangle=\left\langle g_{4}, g_{4}\right\rangle=0 \\
& \left\langle g_{1}, g_{4}\right\rangle=-\left\langle g_{2}, g_{3}\right\rangle=\frac{\tau}{\sqrt{4+5 \tau^{2}+\tau^{4}}}, \\
& \left\langle g_{1}, g_{2}\right\rangle=\left\langle g_{1}, g_{3}\right\rangle=\left\langle g_{2}, g_{4}\right\rangle=\left\langle g_{3}, g_{4}\right\rangle=0,
\end{aligned}
$$

ao passo que (1.17) confere

$$
\begin{aligned}
& \left\langle J_{1} g_{1}, g_{2}\right\rangle=-1, \\
& \left\langle J_{1} g_{2}, g_{4}\right\rangle=\left\langle J_{1} g_{1}, g_{3}\right\rangle=\frac{\tau}{\sqrt{4+5 \tau^{2}+\tau^{4}}}, \\
& \left\langle J_{1} g_{1}, g_{4}\right\rangle=\left\langle J_{1} g_{2}, g_{3}\right\rangle=\left\langle J_{1} g_{3}, g_{4}\right\rangle=0 .
\end{aligned}
$$


Agora, tomando

$$
\left\{\begin{array}{l}
e_{1}=g_{1}, \\
e_{2}=g_{2}, \\
e_{3}=\frac{g_{3}}{\left\langle g_{2}, g_{3}\right\rangle}-g_{2}, \\
e_{4}=\frac{g_{4}}{\left\langle g_{1}, g_{4}\right\rangle}-g_{1},
\end{array}\right.
$$

temos que $\left\{e_{i}\right\}$ é uma base ortonormal de $\mathbb{R}_{2}^{4}$ que satisfaz:

$$
\begin{aligned}
& \left\langle J_{1} e_{1}, e_{2}\right\rangle=\left\langle J_{1} e_{3}, e_{4}\right\rangle=-1, \\
& \left\langle J_{1} e_{1}, e_{3}\right\rangle=\left\langle J_{1} e_{1}, e_{4}\right\rangle=\left\langle J_{1} e_{2}, e_{3}\right\rangle=\left\langle J_{1} e_{2}, e_{4}\right\rangle=0 .
\end{aligned}
$$

Concluímos que $e_{2}=-J_{1} e_{1}$ e $e_{4}=J_{1} e_{3}$. Dessa forma, se considerarmos a base ortonormal $\left\{\tilde{E}_{i}\right\}_{i=1}^{4}$ de $\mathbb{R}_{2}^{4}$ dada por

$$
\tilde{E}_{1}=(1,0,0,0), \quad \tilde{E}_{2}=(0,-1,0,0), \quad \tilde{E}_{3}=(0,0,1,0), \quad \tilde{E}_{4}=(0,0,0,1),
$$

deve haver uma matriz $A \in \mathrm{O}_{2}(4)$, com $J_{1} A=A J_{1}$, tal que $e_{i}=A \tilde{E}_{i}, i \in\{1, \ldots, 4\}$. Finalmente, pondo $\left\langle g_{1}, g_{4}\right\rangle=g_{14}$, podemos reescrever (1.21) como (1.19).

\subsection{0 caso $b^{2}>4 a$}

Teorema 1.9. Seja $\gamma: I \rightarrow \operatorname{SL}(2, \mathbb{R})_{\tau} \subset \mathbb{R}_{2}^{4}$ uma curva bi-harmônica própria parametrizada pelo comprimento de arco, tal que $b^{2}>4 a$. Então existem duas possibilidades:

(i)

$$
b=-\tau^{-1}\left(2+\tau^{2}\right) \cos \vartheta+\sqrt{\left(4+5 \tau^{2}\right) \cos ^{2} \vartheta-4\left(1+\tau^{2}\right)}
$$

$e$

$$
\frac{4\left(1+\tau^{2}\right)}{\left(4+5 \tau^{2}\right)} \leq \cos ^{2} \vartheta<\frac{\left(2+\tau^{2}\right)^{2}}{4+5 \tau^{2}+\tau^{4}}
$$

(ii)

$$
b=-\tau^{-1}\left(2+\tau^{2}\right) \cos \vartheta-\sqrt{\left(4+5 \tau^{2}\right) \cos ^{2} \vartheta-4\left(1+\tau^{2}\right)}
$$


$e$

$$
\frac{4\left(1+\tau^{2}\right)}{\left(4+5 \tau^{2}\right)} \leq \cos ^{2} \vartheta
$$

Em ambos os casos, a expressão de $\gamma$ vista como uma curva de $\mathbb{R}_{2}^{4}$ é

$$
\gamma(s)=A\left(\sqrt{C_{33}} \cos \left(\alpha_{2} s\right), \sqrt{C_{33}} \operatorname{sen}\left(\alpha_{2} s\right), \sqrt{-C_{11}} \cos \left(\alpha_{1} s\right), \sqrt{-C_{11}} \operatorname{sen}\left(\alpha_{1} s\right)\right)
$$

onde

$$
\alpha_{1,2}=\sqrt{\frac{\left(b^{2}-2 a\right) \pm \sqrt{b^{2}\left(b^{2}-4 a\right)}}{2}}
$$

$e$

$$
C_{11}=\frac{\tilde{B}-\alpha_{2}^{2}}{\alpha_{1}^{2}-\alpha_{2}^{2}}, \quad C_{33}=\frac{-\tilde{B}+\alpha_{1}^{2}}{\alpha_{1}^{2}-\alpha_{2}^{2}},
$$

são constantes reais e $A \in \mathrm{O}_{2}(4)$ é uma matriz $4 \times 4$ indefinida e ortogonal que anti-comuta com $J_{1}$.

Demonstração. Primeiro, observamos que a condição $b^{2}>4 a$ nos dá as duas possibilidades (i) e (ii). Além disso, integrando (1.12) obtemos

$$
\gamma(s)=\cos \left(\alpha_{1} s\right) C_{1}+\operatorname{sen}\left(\alpha_{1} s\right) C_{2}+\cos \left(\alpha_{2} s\right) C_{3}+\operatorname{sen}\left(\alpha_{2} s\right) C_{4}
$$

onde

$$
\alpha_{1,2}=\sqrt{\frac{\left(b^{2}-2 a\right) \pm \sqrt{b^{2}\left(b^{2}-4 a\right)}}{2}}
$$

são constantes reais, enquanto $C_{i}, i \in\{1, \ldots, 4\}$, são vetores constantes de $\mathbb{R}_{2}^{4}$.

Pondo $C_{i j}=\left\langle C_{i}, C_{j}\right\rangle$, e avaliando as relações (1.16) em $s=0$, obtemos:

$$
\begin{gathered}
C_{11}+C_{33}+2 C_{13}=1, \\
\alpha_{1}^{2} C_{22}+\alpha_{2}^{2} C_{44}+2 \alpha_{1} \alpha_{2} C_{24}=\tilde{B}, \\
\alpha_{1} C_{12}+\alpha_{2} C_{14}+\alpha_{1} C_{23}+\alpha_{2} C_{34}=0, \\
\alpha_{1}^{3} C_{12}+\alpha_{1} \alpha_{2}^{2} C_{23}+\alpha_{1}^{2} \alpha_{2} C_{14}+\alpha_{2}^{3} C_{34}=0,
\end{gathered}
$$




$$
\begin{gathered}
\alpha_{1}^{4} C_{11}+\alpha_{2}^{4} C_{33}+2 \alpha_{1}^{2} \alpha_{2}^{2} C_{13}=D, \\
\alpha_{1}^{2} C_{11}+\alpha_{2}^{2} C_{33}+\left(\alpha_{1}^{2}+\alpha_{2}^{2}\right) C_{13}=\tilde{B}, \\
\alpha_{1}^{4} C_{22}+\left(\alpha_{1}^{3} \alpha_{2}+\alpha_{1} \alpha_{2}^{3}\right) C_{24}+\alpha_{2}^{4} C_{44}=D, \\
\alpha_{1}^{5} C_{12}+\alpha_{1}^{3} \alpha_{2}^{2} C_{23}+\alpha_{1}^{2} \alpha_{2}^{3} C_{14}+\alpha_{2}^{5} C_{34}=0, \\
\alpha_{1}^{3} C_{12}+\alpha_{1}^{3} C_{23}+\alpha_{2}^{3} C_{14}+\alpha_{2}^{3} C_{34}=0, \\
\alpha_{1}^{6} C_{22}+\alpha_{2}^{6} C_{44}+2 \alpha_{1}^{3} \alpha_{2}^{3} C_{24}=E .
\end{gathered}
$$

De (1.25), (1.26), (1.30), (1.31), segue que

$$
C_{12}=C_{14}=C_{23}=C_{34}=0 \text {. }
$$

Também, de (1.23), 1.27) e (1.28), obtemos

$$
C_{11}=\frac{\tilde{B}-\alpha_{2}^{2}}{\alpha_{1}^{2}-\alpha_{2}^{2}}, \quad C_{13}=0, \quad C_{33}=\frac{-\tilde{B}+\alpha_{1}^{2}}{\alpha_{1}^{2}-\alpha_{2}^{2}} .
$$

Observamos que um cálculo direto nos dá

$$
\alpha_{1}^{2}-\alpha_{2}^{2}=b \sqrt{b^{2}-4 a},
$$

que, claramente, é uma constante positiva. Ademais, não é difícil verificar a validade de

$$
\tilde{B}+\alpha_{2}^{2}=W_{1}+(2 \tau)^{-1} b \sqrt{W_{2}}, \quad \text { e } \quad-\tilde{B}+\alpha_{1}^{2}=-W_{1}+(2 \tau)^{-1} b \sqrt{W_{2}},
$$

onde

$$
W_{1}=-b\left(\tau^{-1} \cos \vartheta+\frac{b}{2}\right) \quad \text { e } \quad W_{2}=(b \tau+2 \cos \vartheta)^{2}-4 \tau^{2} \operatorname{sen}^{2} \vartheta,
$$

Dessa forma, usando fato que $\left|(2 \tau)^{-1} b \sqrt{W_{2}}\right|>\left|W_{1}\right|$ com a condição

$$
\frac{4\left(1+\tau^{2}\right)}{\left(4+5 \tau^{2}\right)} \leq \cos ^{2} \vartheta
$$

podemos garantir que $C_{11}<0$ e $C_{33}>0$. 
Finalmente, usando (1.24), (1.29) e (1.32), concluímos que

$$
C_{22}=\frac{D-\tilde{B} \alpha_{2}^{2}}{\alpha_{1}^{2}\left(\alpha_{1}^{2}-\alpha_{2}^{2}\right)}, \quad C_{24}=0, \quad C_{44}=\frac{-D+\tilde{B} \alpha_{1}^{2}}{\alpha_{2}^{2}\left(\alpha_{1}^{2}-\alpha_{2}^{2}\right)} .
$$

Chamamos a atenção do leitor para o fato de que, utilizando (1.27) e (1.28) juntamente com a informação $C_{13}=0$, podemos obter $C_{11}=C_{22}$ e $C_{33}=C_{44}$.

Como $\left\{C_{i}\right\}_{i=1}^{4}$ são ortogonais entre si e

$$
\left\|C_{1}\right\|=\left\|C_{2}\right\|=\sqrt{-C_{11}}, \quad\left\|C_{3}\right\|=\left\|C_{4}\right\|=\sqrt{C_{33}},
$$

fazendo $e_{i}=C_{i} /\left\|C_{i}\right\|, i \in\{1, \ldots, 4\}$ obtemos uma base pseudo-ortonormal de $\mathbb{R}_{2}^{4}$, e podemos escrever:

$$
\gamma(s)=\sqrt{-C_{11}}\left(\cos \left(\alpha_{1} s\right) e_{1}+\operatorname{sen}\left(\alpha_{1} s\right) e_{2}\right)+\sqrt{C_{33}}\left(\cos \left(\alpha_{2} s\right) e_{3}+\operatorname{sen}\left(\alpha_{2} s\right) e_{4}\right) .
$$

Agora, avaliando em $s=0$ as identidades (1.17), temos:

$$
\begin{gathered}
\alpha_{2} C_{33}\left\langle J_{1} e_{3}, e_{4}\right\rangle-\alpha_{1} C_{11}\left\langle J_{1} e_{1}, e_{2}\right\rangle \\
+\sqrt{-C_{11} C_{33}}\left(\alpha_{1}\left\langle J_{1} e_{3}, e_{2}\right\rangle+\alpha_{2}\left\langle J_{1} e_{1}, e_{4}\right\rangle\right)=-\tau^{-1} \cos \vartheta \\
\left\langle J_{1} e_{1}, e_{3}\right\rangle=0, \\
\alpha_{2}^{3} C_{33}\left\langle J_{1} e_{3}, e_{4}\right\rangle-\alpha_{1}^{3} C_{11}\left\langle J_{1} e_{1}, e_{2}\right\rangle \\
+\sqrt{-C_{11} C_{33}}\left(\alpha_{1} \alpha_{2}^{2}\left\langle J_{1} e_{3}, e_{2}\right\rangle+\alpha_{1}^{2} \alpha_{2}\left\langle J_{1} e_{1}, e_{4}\right\rangle\right)=-I, \\
\left\langle J_{1} e_{2}, e_{4}\right\rangle=0 \\
\alpha_{1}\left\langle J_{1} e_{2}, e_{3}\right\rangle+\alpha_{2}\left\langle J_{1} e_{1}, e_{4}\right\rangle=0 \\
\alpha_{2}\left\langle J_{1} e_{2}, e_{3}\right\rangle+\alpha_{1}\left\langle J_{1} e_{1}, e_{4}\right\rangle=0 .
\end{gathered}
$$

Observamos que para obter as identidades acima, fizemos divisões por $\alpha_{1}^{2}-\alpha_{2}^{2}=\sqrt{b^{2}\left(b^{2}-4 a\right)}$, que é diferente de zero. De (1.34) e (1.35), levando em conta que $\alpha_{1}^{2}-\alpha_{2}^{2} \neq 0$, resulta que

$$
\left\langle J_{1} e_{3}, e_{2}\right\rangle=0, \quad\left\langle J_{1} e_{1}, e_{4}\right\rangle=0 .
$$


Então,

$$
J_{1} e_{1}= \pm e_{2} \quad \text { e } \quad J_{1} e_{3}= \pm e_{4} .
$$

Portanto, o vetor posição de $\gamma$ é dado por

$$
\gamma(s)=\sqrt{-C_{11}}\left(\cos \left(\alpha_{1} s\right) e_{1} \pm \operatorname{sen}\left(\alpha_{1} s\right) J_{1} e_{1}\right)+\sqrt{C_{33}}\left(\cos \left(\alpha_{2} s\right) e_{3} \pm \operatorname{sen}\left(\alpha_{2} s\right) J_{1} e_{3}\right)
$$

De (1.14) para $s=0$, vemos que $J_{1} e_{1}=-e_{2}$ e $J_{1} e_{3}=-e_{4}$. Então, se fixarmos a base ortonormal de $\mathbb{R}_{2}^{4}$ dada por

$$
\bar{E}_{1}=(0,0,1,0), \quad \bar{E}_{2}=(0,0,0,1), \quad \bar{E}_{3}=(1,0,0,0), \quad \bar{E}_{4}=(0,1,0,0),
$$

deve haver uma matriz $A \in \mathrm{O}_{2}(4)$, com $J_{1} A=-A J_{1}$, tal que $e_{i}=A \bar{E}_{i}, i \in\{1,2,3,4\}$. Substituindo $e_{i}=A \bar{E}_{i}$ em (1.33) obtemos (1.22).

\subsection{0 caso $b^{2}<4 a$}

Teorema 1.10. Seja $\gamma: I \rightarrow \operatorname{SL}(2, \mathbb{R})_{\tau} \subset \mathbb{R}_{2}^{4}$ uma curva bi-harmônica parametrizada pelo comprimento de arco, tal que $b^{2}<4 a$. Então

$$
\begin{gathered}
b=-\tau^{-1}\left(2+\tau^{2}\right) \cos \vartheta+\sqrt{\left(4+5 \tau^{2}\right) \cos ^{2} \vartheta-4\left(1+\tau^{2}\right)}, \\
\frac{\left(2+\tau^{2}\right)^{2}}{4+5 \tau^{2}+\tau^{4}}<\cos ^{2} \vartheta<1,
\end{gathered}
$$

e a expressão de $\gamma$ vista como uma curva de $\mathbb{R}_{2}^{4}$ é dada por:

$$
\begin{aligned}
\gamma(s)= & A\left(\cos \left(\frac{b}{2} s\right) \cosh (\mu s)+w_{14} \operatorname{sen}\left(\frac{b}{2} s\right) \operatorname{senh}(\mu s),\right. \\
& \operatorname{sen}\left(\frac{b}{2} s\right) \cosh (\mu s)-w_{14} \cos \left(\frac{b}{2} s\right) \operatorname{senh}(\mu s), \\
& \left.\cos \left(\frac{b}{2} s\right) \operatorname{senh}(\mu s) \sqrt{1+w_{14}^{2}}, \operatorname{sen}\left(\frac{b}{2} s\right) \operatorname{senh}(\mu s) \sqrt{1+w_{14}^{2}}\right),
\end{aligned}
$$

onde

$$
\mu=\frac{\sqrt{4 a-b^{2}}}{2}, \quad w_{14}=\frac{b \tau+2 \cos \vartheta}{2 \tau \mu}
$$

são constantes reais e $A \in \mathrm{O}_{2}(4)$ é uma matriz $4 \times 4$ ortogonal indefinida que comuta com $J_{1}$. 
Demonstração. De $b^{2}<4 a$, resulta que $b$ é dada por (1.36) e $\vartheta$ satisfaz (1.37). Além disso, integrando (1.12), obtemos

$$
\gamma(s)=\cos \left(\frac{b}{2} s\right)\left(\cosh (\mu s) w_{1}+\operatorname{senh}(\mu s) w_{3}\right)+\operatorname{sen}\left(\frac{b}{2} s\right)\left(\cosh (\mu s) w_{2}+\operatorname{senh}(\mu s) w_{4}\right),
$$

onde

$$
\mu=\frac{\sqrt{4 a-b^{2}}}{2}
$$

enquanto $w_{i}, i \in\{1, \ldots, 4\}$, são vetores constantes de $\mathbb{R}_{2}^{4}$. Se $w_{i j}:=\left\langle w_{i}, w_{j}\right\rangle$, avaliando as relações (1.16) em $s=0$, obtemos

$$
\begin{aligned}
& w_{11}=1, \\
& \frac{b^{2}}{4} w_{22}+\mu^{2} w_{33}+\mu b w_{23}=\tilde{B}, \\
& \frac{b}{2} w_{12}+\mu w_{13}=0 \\
& \frac{b}{2}\left(\mu^{2}-\frac{b^{2}}{4}\right) w_{12}+\mu^{2} b w_{34}+\mu \frac{b^{2}}{2} w_{24}+\mu\left(\mu^{2}-\frac{b^{2}}{4}\right) w_{13}=0, \\
& \left(\mu^{2}-\frac{b^{2}}{4}\right)^{2} w_{11}+\mu^{2} b^{2} w_{44}+2 \mu b\left(\mu^{2}-\frac{b^{2}}{4}\right) w_{14}=D \\
& \left(\mu^{2}-\frac{b^{2}}{4}\right) w_{11}+\mu b w_{14}=-\tilde{B} \\
& \frac{b^{2}}{4}\left(3 \mu^{2}-\frac{b^{2}}{4}\right) w_{22}+\mu^{2}\left(\mu^{2}-3 \frac{b^{2}}{4}\right) w_{33}+\mu \frac{b}{2}\left(4 \mu^{2}-b^{2}\right) w_{23}=-D, \\
& \frac{b}{2}\left(3 \mu^{2}-\frac{b^{2}}{4}\right)\left(\mu^{2}-\frac{b^{2}}{4}\right) w_{12}+b \mu^{2}\left(\mu^{2}-3 \frac{b^{2}}{4}\right) w_{34} \\
& +\mu\left(\mu^{2}-3 \frac{b^{2}}{4}\right)\left(\mu^{2}-\frac{b^{2}}{4}\right) w_{13}+\mu \frac{b^{2}}{2}\left(3 \mu^{2}-\frac{b^{2}}{4}\right) w_{24}=0, \\
& \frac{b}{2}\left(3 \mu^{2}-\frac{b^{2}}{4}\right) w_{12}+\mu\left(\mu^{2}-3 \frac{b^{2}}{4}\right) w_{13}=0, \\
& \frac{b^{2}}{4}\left(3 \mu^{2}-\frac{b^{2}}{4}\right)^{2} w_{22}+\mu^{2}\left(\mu^{2}-3 \frac{b^{2}}{4}\right)^{2} w_{33} \\
& +\mu b\left(3 \mu^{2}-\frac{b^{2}}{4}\right)\left(\mu^{2}-3 \frac{b^{2}}{4}\right) w_{23}=E .
\end{aligned}
$$

De (1.40), 1.44) e (1.45), segue que

$$
w_{11}=-w_{44}=1, \quad w_{14}=\frac{b \tau+2 \cos \vartheta}{2 \tau \mu} .
$$


Além disso, de (1.42) e (1.48), obtemos que

$$
w_{12}=w_{13}=0
$$

e, portanto, de (1.43) e (1.47),

$$
w_{24}=w_{34}=0 \text {. }
$$

Usando também (1.41), (1.46) e (1.49), temos

$$
w_{22}=-w_{33}=1, \quad w_{23}=-\frac{b \tau+2 \cos \vartheta}{2 \tau \mu} .
$$

Então, podemos definir a seguinte base pseudo-ortonormal em $\mathbb{R}_{2}^{4}$ :

$$
\left\{\begin{array}{l}
e_{1}=w_{1} \\
e_{2}=w_{2} \\
e_{3}=\frac{w_{3}+w_{14} w_{2}}{\sqrt{1+w_{14}^{2}}} \\
e_{4}=\frac{w_{4}-w_{14} w_{1}}{\sqrt{1+w_{14}^{2}}}
\end{array}\right.
$$

$\operatorname{com}\left\langle e_{1}, e_{1}\right\rangle=1=\left\langle e_{2}, e_{2}\right\rangle$ e $\left\langle e_{3}, e_{3}\right\rangle=-1=\left\langle e_{4}, e_{4}\right\rangle$.

Avaliando as identidades (1.17) em $s=0$, e levando em conta que:

$$
\begin{aligned}
& \gamma(0)=w_{1}, \\
& \gamma^{\prime}(0)=\frac{b}{2} w_{2}+\mu w_{3}, \\
& \gamma^{\prime \prime}(0)=\left(\mu^{2}-\frac{b^{2}}{4}\right) w_{1}+\mu b w_{4}, \\
& \gamma^{\prime \prime \prime}(0)=\frac{b}{2}\left(3 \mu^{2}-\frac{b^{2}}{4}\right) w_{2}+\mu\left(\mu^{2}-\frac{3}{4} b^{2}\right) w_{3}, \\
& \gamma^{I V}(0)=\left(\mu^{4}-\frac{3}{2} \mu^{2} b^{2}+\frac{b^{4}}{16}\right) w_{1}+2 \mu b\left(\mu^{2}-\frac{b^{2}}{4}\right) w_{4},
\end{aligned}
$$

temos

$$
\begin{aligned}
& \left\langle J_{1} w_{1}, w_{2}\right\rangle=-\left\langle J_{1} w_{3}, w_{4}\right\rangle=1, \\
& \left\langle J_{1} w_{3}, w_{2}\right\rangle=\left\langle J_{1} w_{1}, w_{4}\right\rangle=0, \\
& \left\langle J_{1} w_{1}, w_{3}\right\rangle=\left\langle J_{1} w_{2}, w_{4}\right\rangle=-w_{14} .
\end{aligned}
$$


Então,

$$
\begin{gathered}
\left\langle J_{1} e_{1}, e_{2}\right\rangle=-\left\langle J_{1} e_{3}, e_{4}\right\rangle=1, \\
\left\langle J_{1} e_{1}, e_{4}\right\rangle=\left\langle J_{1} e_{1}, e_{3}\right\rangle=\left\langle J_{1} e_{2}, e_{3}\right\rangle=\left\langle J_{1} e_{2}, e_{4}\right\rangle=0 .
\end{gathered}
$$

Portanto, concluímos que

$$
J_{1} e_{1}=e_{2}, \quad J_{1} e_{3}=e_{4}
$$

Consequentemente, se considerarmos a base ortonormal $\left\{E_{i}\right\}_{i=1}^{4}$ de $\mathbb{R}_{2}^{4}$ dada por

$$
E_{1}=(1,0,0,0), \quad E_{2}=(0,1,0,0), \quad E_{3}=(0,0,1,0), \quad E_{4}=(0,0,0,1),
$$

deve existir uma matriz $A \in \mathrm{O}_{2}(4), \operatorname{com} J_{1} A=A J_{1}$, tal que $e_{i}=A E_{i}, i \in\{1,2,3,4\}$. Portanto, usando (1.39) e (1.50) obtemos (1.38). 



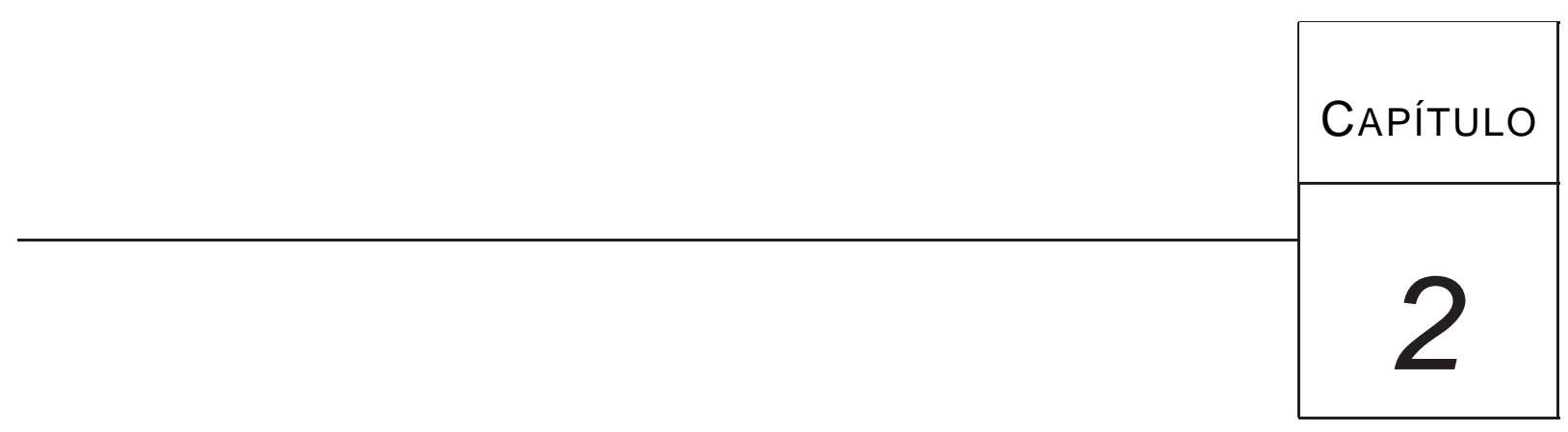

\section{Superfícies biconservativas em}

BCV-espaços

\subsection{Espaços de Bianchi-Cartan-Vranceanu e sua estru- tura Riemanniana}

É um resultado clássico da geometria diferencial (veja, por exemplo, [67]) que a dimensão máxima do grupo das isometrias de uma variedade Riemanniana tridimensional é 6 e, nesse caso, a variedade é uma forma espacial. Alpem disso, sabe-se que não existem 3-variedades Riemannianas 
com grupo de isometrias de dimensão 5 (veja [35]). e que se uma 3-variedade Riemanniana possui grupo de isometrias 4-dimensional, então é uma variedade homogênea.

Para as variedades Riemannianas homogêneas com grupo de isometria de dimensão 4 e para aquelas com curvatura seccional constante não negativa, existe uma representação local dada pela seguinte família a dois parâmetros de métricas Riemannianas:

$$
g_{\kappa, \tau}=\frac{d x^{2}+d y^{2}}{F^{2}}+\left(d z+\tau \frac{y d x-x d y}{F}\right)^{2},
$$

onde $F=1+\frac{\kappa}{4}\left(x^{2}+y^{2}\right)$ e $\kappa, \tau \in \mathbb{R} ;$ definidas em $\mathcal{N}=\mathbb{R}^{3}$ se $\kappa \geq 0$, senão em

$$
\mathcal{N}=\left\{(x, y, z) \in \mathbb{R}^{3}: x^{2}+y^{2}<-4 / \kappa\right\}
$$

As métricas $g_{\kappa, \tau}$, denominadas métricas de Bianchi-Cartan-Vranceanu, podem ser encontradas na classificação das variedades Riemannianas homogêneas tridimensionais dada por L. Bianchi em 1897 (veja [4]) e, mais tarde, aparecem na forma (2.1) em [15,67], com É. Cartan e G. Vranceanu (respectivamente). No que segue, denotaremos $\operatorname{com} \mathcal{N}_{\kappa, \tau}$ o espaço $\mathcal{N}$ munido da métrica $g_{\kappa, \tau}$.

A importância e o interesse na nesta família de métricas está no fato dela incluir todas as métricas homogêneas tridimensionais cujo grupo de isometrias é de dimensão 4 ou 6 , exceto às de curvatura seccional constante negativa. Indicando com $\mathbb{H}^{2}$ o plano hiperbólico de raio $1 /(\sqrt{-k})$ e com $\mathbb{S}^{2}$ a esfera de $\mathbb{R}^{3}$ de raio $1 /(\sqrt{k})$, temos a situação ilustrada na Figura 2.1.

Observe que:

- se $4 \tau^{2}=\kappa$, então $\mathcal{N}_{\kappa, \tau}$ possui curvatura seccional constante e não negativa, ou seja $\mathcal{N}_{\kappa, \tau}$ é o espaço Euclidiano $\mathbb{R}^{3}(\kappa=0)$ ou a esfera $\mathbb{S}^{3}(\kappa \neq 0)$;

- se $\tau=0$, então $\mathcal{N}_{\kappa, \tau}$ é produto de uma superfície de curvatura Gaussiana constante $\kappa$ com a reta real $\mathbb{R}$;

- se $\tau \neq 0$ e $\kappa>0, \mathcal{N}_{\kappa, \tau}$ é localmente uma esfera de Berger; 


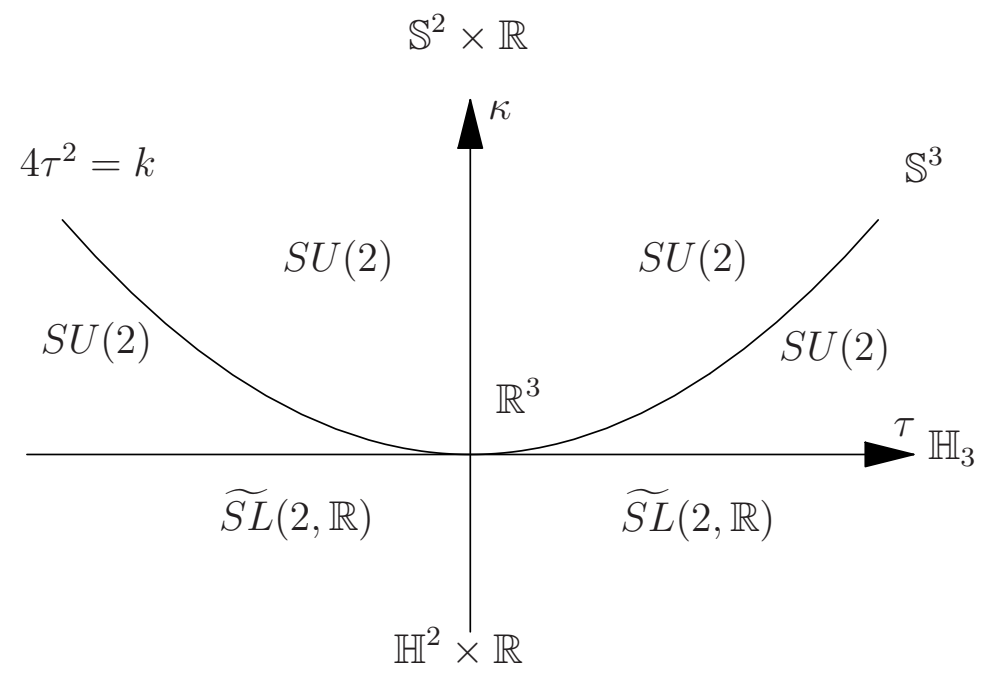

Figura 2.1: Descrição geométrica da métrica $g_{\kappa, \tau}$ (veja [62]).

- se $\tau \neq 0$ e $\kappa<0, \mathcal{N}_{\kappa, \tau}$ é localmente o recobrimento universal do grupo linear especial, i.e. $\widetilde{S L}(2, \mathbb{R})$

- se $\tau \neq 0$ e $\kappa=0$, obtemos o espaço de Heisenberg $\mathbb{H}_{3}$ munido de uma métrica invariante à esquerda.

Para que possamos fazer nosso estudo nesses espaços, precisamos conhecer sua estrutura Riemanniana, que passamos a descrever. A métrica de Bianchi-Cartan-Vranceanu pode ser escrita através de 1-formas, como

$$
d s_{\kappa, \tau}^{2}=\sum_{i=1}^{3} w_{i} \otimes w_{i}
$$

onde

$$
w_{1}=d z+\tau \frac{y d x-x d y}{F} ; \quad w_{2}=\frac{d x}{F} ; \quad w_{3}=\frac{d y}{F}
$$

A base ortonormal de campos de vetores correspondentes às 1-formas é dada por

$$
E_{1}=\frac{\partial}{\partial z}, \quad E_{2}=F \frac{\partial}{\partial x}-\tau y \frac{\partial}{\partial z}, \quad E_{3}=F \frac{\partial}{\partial y}+\tau x \frac{\partial}{\partial z}
$$

Quanto ao grupo de isometrias do espaço $\mathcal{N}_{\kappa, \tau}$ temos a proposição que segue. 
Proposição 2.1 ( [62]]). A álgebra de Lie das isometrias infinitesimais de $\mathcal{N}_{\kappa, \tau}$ admite como base

$$
\left\{\begin{array}{l}
X_{1}=\left(1-\frac{\kappa y^{2}}{2 F}\right) E_{1}+\frac{\kappa x y}{2 F} E_{2}+\frac{2 \tau y}{F} E_{3} \\
X_{2}=\frac{\kappa x y}{2 F} E_{1}+\left(1-\frac{\kappa x^{2}}{2 F}\right) E_{2}-\frac{2 \tau x}{F} E_{3} \\
X_{3}=\frac{-y}{F} E_{1}+\frac{x}{F} E_{2}-\frac{\tau\left(x^{2}+y^{2}\right)}{F} E_{3} \\
X_{4}=E_{3} .
\end{array}\right.
$$

Além disso, as expressões da conexão de Levi-Civita, com respeito a base ortonormal (2.2), é dada por:

$$
\begin{array}{l|l|l}
\bar{\nabla}_{E_{1}} E_{1}=0, & \bar{\nabla}_{E_{2}} E_{2}=\frac{\kappa}{2} y E_{3}, \\
\bar{\nabla}_{E_{2}} E_{1}=\bar{\nabla}_{E_{1}} E_{2}=-\tau E_{3}, & \bar{\nabla}_{E_{3}} E_{2}=-\frac{\kappa}{2} x E_{3}-\tau E_{1}, \\
\bar{\nabla}_{E_{1}} E_{3}=\bar{\nabla}_{E_{3}} E_{1}=\tau E_{2} . & \bar{\nabla}_{E_{2}} E_{3}=-\frac{\kappa}{2} y E_{2}+\tau E_{1}, & \bar{\nabla}_{E_{3}} E_{3}=\frac{\kappa}{2} x E_{2},
\end{array}
$$

Chamamos a atenção do leitor para as seguintes notações e convenção de sinal que adotaremos. O operador de curvatura possui a expressão

$$
\mathrm{R}(X, Y) Z=\bar{\nabla}_{Y} \bar{\nabla}_{X} Z-\bar{\nabla}_{X} \bar{\nabla}_{Y} Z+\bar{\nabla}_{[X, Y]} Z
$$

onde $X, Y, Z$ são campos de vetores diferenciáveis em $\mathcal{N}_{\kappa, \tau}$. Dessa forma temos que

$$
\begin{aligned}
& g_{\kappa, \tau}\left(\mathrm{R}\left(E_{1}, E_{2}\right) E_{1}, E_{2}\right)=\tau^{2}, \quad g_{\kappa, \tau}\left(\mathrm{R}\left(E_{1}, E_{3}\right) E_{1}, E_{3}\right)=\tau^{2} \\
& g_{\kappa, \tau}\left(\mathrm{R}\left(E_{2}, E_{3}\right) E_{2}, E_{3}\right)=\kappa-3 \tau^{2} .
\end{aligned}
$$

Podemos, então, calcular as componentes não nulas da curvatura de Ricci, as quais denotaremos por $\operatorname{Ricci}_{i j}$. Resulta que:

$$
\operatorname{Ricci}\left(E_{1}, E_{1}\right)=2 \tau^{2}, \quad \operatorname{Ricci}\left(E_{2}, E_{2}\right)=\operatorname{Ricci}\left(E_{3}, E_{3}\right)=\kappa-2 \tau^{2} .
$$

\subsection{Equações de compatibilidade}

Um fato importante dos BCV-espaços é que eles admitem uma submersão Riemanniana sobre uma superfície de curvatura Gaussiana constante $\kappa$, chamada fibração de Hopf. O campo de vetores 
tangente às fibras dessa projeção é o campo de Killing $E_{1}$, também conhecido como campo de Hopf.

Em [18], Daniel estudou as superfícies localmente isometricamente imersas em variedades Riemannianas tridimensionais homogêneas com grupo de isometria de dimensão 4, que, como vimos na seção anterior, são os espaços de Bianchi-Cartan-Vranceanu que cumprem $4 \tau^{2} \neq \kappa$. Neste estudo, o autor considera o ângulo $\vartheta$ que o campo de vetores unitário normal à superfície faz com o campo de Hopf, provando que as equações de Gauss e Codazzi são dadas em termos da função $\vartheta$ e que este ângulo é um dos invariantes fundamentais para uma superfície em BCVespaços.

No que segue, apresentaremos alguns resultados de [18] que nos serão úteis mais tarde.

Começamos considerando uma superfície $\mathcal{M}$ simplesmente conexa e orientada em $\mathcal{N}_{\kappa, \tau}$, denotamos com $\nabla$ sua conexão de Levi-Civita, $N$ o campo de vetores unitário normal a $\mathcal{M}, A$ o operador de forma associado ao campo $N, K$ a curvatura Gaussiana da superfície e $B(X, Y):=$ $\bar{\nabla}_{X} Y-\nabla_{X} Y, \operatorname{com} X, Y \in T M$, a segunda forma fundamental. Isto posto, vale

$$
\left|g_{\kappa, \tau}\left(E_{1}, N\right)\right|=\cos \vartheta, \quad \vartheta \in[0, \pi / 2]
$$

Projetando $E_{1}$ no plano tangente a $\mathcal{M}$ obtemos

$$
E_{1}=T+\cos \vartheta N
$$

onde $T$ é a parte tangente e satisfaz $g_{\kappa, \tau}(T, T)=\operatorname{sen}^{2} \vartheta$.

No que segue, usaremos $\mathbb{E}$ para denotar os BCV-espaços com grupo de isometria de dimensão 4. Temos os resultados que seguem.

Proposição 2.2 ( [18]). Para quaisquer campos de vetores $X, Y, Z, W$ em $\mathbb{E}$, temos:

$$
g_{\kappa, \tau}(\mathrm{R}(X, Y) Z, W)=\left(\kappa-3 \tau^{2}\right) g_{\kappa, \tau}\left(\mathrm{R}_{0}(X, Y) Z, W\right)+\left(\kappa-4 \tau^{2}\right) g_{\kappa, \tau}\left(\mathrm{R}_{1}\left(E_{1} ; X, Y\right) Z, W\right),
$$

com

$$
\mathrm{R}_{0}(X, Y) Z=g_{\kappa, \tau}(X, Z) Y-g_{\kappa, \tau}(Y, Z) X
$$




$$
\begin{aligned}
\mathrm{R}_{1}(V ; X, Y) Z= & g_{\kappa, \tau}(Y, V) g_{\kappa, \tau}(Z, V) X+g_{\kappa, \tau}(Y, Z) g_{\kappa, \tau}(X, V) V \\
& -g_{\kappa, \tau}(X, Z) g_{\kappa, \tau}(Y, V) V-g_{\kappa, \tau}(X, V) g_{\kappa, \tau}(Z, V) Y
\end{aligned}
$$

Demonstração. Escrevemos $X=\tilde{X}+x E_{1}$, com $\widetilde{X}$ horizontal com relação à fibração de Hopf e $x=g_{\kappa, \tau}\left(X, E_{1}\right)$, e analogamente faremos para os campos $Y, Z, W$. Usando a multilinearidade do tensor de curvatura, obtemos uma soma de 16 termos; os termos em que $E_{1}$ aparece três vezes ou quatro vezes, ou duas nas posições 1,2 ou 3,4 , se anulam devido à antissimetria do tensor. Os termos em que $E_{1}$ aparece uma única vez se anulam, já que nas componentes não nulas do tensor curvatura, (2.4), $E_{1}$ aparece duas vezes ou nenhuma. Portanto, temos

$$
\begin{aligned}
g_{\kappa, \tau}(\mathrm{R}(X, Y) Z, W)= & g_{\kappa, \tau}(\mathrm{R}(\widetilde{X}, \widetilde{Y}) \widetilde{Z}, \widetilde{W})+y w g_{\kappa, \tau}\left(\mathrm{R}\left(\widetilde{X}, E_{1}\right) \widetilde{Z}, E_{1}\right) \\
& +y z g_{\kappa, \tau}\left(\mathrm{R}\left(\widetilde{X}, E_{1}\right) E_{1}, \widetilde{W}\right)+x w g_{\kappa, \tau}\left(\mathrm{R}\left(E_{1}, \widetilde{Y}\right) \widetilde{Z}, E_{1}\right) \\
& +x z g_{\kappa, \tau}\left(\mathrm{R}\left(E_{1}, \widetilde{Y}\right) E_{1}, \widetilde{W}\right) \\
= & \left(\kappa-3 \tau^{2}\right)\left[g_{\kappa, \tau}(\widetilde{X}, \widetilde{Z}) g_{\kappa, \tau}(\widetilde{Y}, \widetilde{W})-g_{\kappa, \tau}(\widetilde{X}, \widetilde{W}) g_{\kappa, \tau}(\widetilde{Y}, \widetilde{Z})\right] \\
& +\tau^{2}\left[y w g_{\kappa, \tau}(\widetilde{X}, \widetilde{Z})-y z g_{\kappa, \tau}(\widetilde{X}, \widetilde{W})-x w g_{\kappa, \tau}(\widetilde{Y}, \widetilde{Z})\right. \\
& \left.+x z g_{\kappa, \tau}(\widetilde{Y}, \widetilde{W})\right] .
\end{aligned}
$$

Neste ponto convém observar que

$$
g_{\kappa, \tau}(\tilde{X}, \widetilde{Z})=g_{\kappa, \tau}\left(X-x E_{1}, Z-z E_{1}\right)=g_{\kappa, \tau}(X, Z)-x z,
$$

e analogamente para os demais campos. Desta forma temos

$$
\begin{aligned}
{\left[g_{\kappa, \tau}(\widetilde{X}, \widetilde{Z}) g_{\kappa, \tau}(\widetilde{Y}, \widetilde{W})-g_{\kappa, \tau}(\widetilde{X}, \widetilde{W}) g_{\kappa, \tau}(\widetilde{Y}, \widetilde{Z})\right]=} \\
\quad\left[g_{\kappa, \tau}(X, Z) g_{\kappa, \tau}(Y, W)-g_{\kappa, \tau}(X, W) g_{\kappa, \tau}(Y, Z)\right] \\
\quad-\left[g_{\kappa, \tau}(X, Z) g_{\kappa, \tau}\left(Y, E_{1}\right) g_{\kappa, \tau}\left(W, E_{1}\right)+g_{\kappa, \tau}(Y, W) g_{\kappa, \tau}\left(X, E_{1}\right) g_{\kappa, \tau}\left(Z, E_{1}\right)\right. \\
\left.-g_{\kappa, \tau}(X, W) g_{\kappa, \tau}\left(Y, E_{1}\right) g_{\kappa, \tau}\left(Z, E_{1}\right)-g_{\kappa, \tau}(Y, Z) g_{\kappa, \tau}\left(X, E_{1}\right) g_{\kappa, \tau}\left(W, E_{1}\right)\right],
\end{aligned}
$$

$\mathrm{e}$

$$
\begin{aligned}
{\left[y w g_{\kappa, \tau}(\widetilde{X}, \widetilde{Z})-\right.} & \left.y z g_{\kappa, \tau}(\widetilde{X}, \widetilde{W})-x w g_{\kappa, \tau}(\widetilde{Y}, \widetilde{Z})+x z g_{\kappa, \tau}(\widetilde{Z}, \widetilde{W})\right]= \\
& {\left[g_{\kappa, \tau}(X, Z) g_{\kappa, \tau}\left(Y, E_{1}\right) g_{\kappa, \tau}\left(W, E_{1}\right)+g_{\kappa, \tau}(Y, W) g_{\kappa, \tau}\left(X, E_{1}\right) g_{\kappa, \tau}\left(Z, E_{1}\right)\right.} \\
& \left.-g_{\kappa, \tau}(X, W) g_{\kappa, \tau}\left(Y, E_{1}\right) g_{\kappa, \tau}\left(Z, E_{1}\right)-g_{\kappa, \tau}(Y, Z) g_{\kappa, \tau}\left(X, E_{1}\right) g_{\kappa, \tau}\left(W, E_{1}\right)\right] .
\end{aligned}
$$


Concluímos, assim, que

$$
\begin{aligned}
g_{\kappa, \tau}(\mathrm{R}(X, Y) Z, W)= & \left.\kappa-3 \tau^{2}\right)\left[g_{\kappa, \tau}(X, Z) g_{\kappa, \tau}(Y, W)-g_{\kappa, \tau}(X, W) g_{\kappa, \tau}(Y, Z)\right] \\
& -\left(\kappa-4 \tau^{2}\right)\left[g_{\kappa, \tau}(X, Z) g_{\kappa, \tau}\left(Y, E_{1}\right) g_{\kappa, \tau}\left(W, E_{1}\right)\right. \\
& +g_{\kappa, \tau}(Y, W) g_{\kappa, \tau}\left(X, E_{1}\right) g_{\kappa, \tau}\left(Z, E_{1}\right)-g_{\kappa, \tau}(X, W) g_{\kappa, \tau}\left(Y, E_{1}\right) g_{\kappa, \tau}\left(Z, E_{1}\right) \\
& \left.-g_{\kappa, \tau}(Y, Z) g_{\kappa, \tau}\left(X, E_{1}\right) g_{\kappa, \tau}\left(W, E_{1}\right)\right] .
\end{aligned}
$$

Proposição 2.3 ( [18]). Sejam $X, Y, Z, W$ campos de vetores tangentes a $\mathcal{M} \subset \mathbb{E}$. Então,

$$
\begin{gathered}
g_{\kappa, \tau}(\mathrm{R}(X, Y) Z, W)=\left(\kappa-3 \tau^{2}\right) g_{\kappa, \tau}\left(\mathrm{R}_{0}(X, Y) Z, W\right)+\left(\kappa-4 \tau^{2}\right) g_{\kappa, \tau}\left(\mathrm{R}_{1}(T ; X, Y) Z, W\right), \\
\mathrm{R}(X, Y) N=\left(\kappa-4 \tau^{2}\right) \cos \vartheta\left(g_{\kappa, \tau}(Y, T) X-g_{\kappa, \tau}(X, T) Y\right) .
\end{gathered}
$$

Demonstração. O resultado é uma consequência da Proposição 2.2, usando o fato de que $X, Y, Z$ e $W$ são tangentes a superfície e $N$ é normal.

Proposição 2.4 ( [18]). As equações de Gauss e Codazzi em um espaço homogêneo tridimensional $\mathcal{N}_{\kappa, \tau}$ com grupo de isometrias de dimensão 4 são, respectivamente, dadas por:

$$
\begin{gathered}
K=\operatorname{det} A+\tau^{2}+\left(\kappa-4 \tau^{2}\right) \cos ^{2} \vartheta \\
\nabla_{X} A Y-\nabla_{Y} A X-A[X, Y]=\left(\kappa-4 \tau^{2}\right) \cos \vartheta\left(g_{\kappa, \tau}(Y, T) X-g_{\kappa, \tau}(X, T) Y\right),
\end{gathered}
$$

sendo $X, Y$ campos de vetores tangentes a $\mathcal{M}$.

Demonstração. Começamos com a equação de Gauss, que pode ser escrita na forma

$$
K=\operatorname{det} A+g_{\kappa, \tau}(\mathrm{R}(X, Y) X, Y),
$$

onde $X$ e $Y$ formam uma base ortonormal de $T \mathcal{M}$. Usando a Proposição 2.3 obtemos

$$
g_{\kappa, \tau}(\mathrm{R}(X, Y) X, Y)=\left(\kappa-3 \tau^{2}\right) g_{\kappa, \tau}\left(\mathrm{R}_{0}(X, Y) X, Y\right)+\left(\kappa-4 \tau^{2}\right) g_{\kappa, \tau}\left(\mathrm{R}_{1}(T ; X, Y) X, Y\right),
$$


sendo que, por cálculos diretos e usando a ortonormalidade de $\{X, Y\}$, temos

$$
g_{\kappa, \tau}\left(\mathrm{R}_{0}(X, Y) X, Y\right)=1 \quad \text { e } \quad g_{\kappa, \tau}\left(\mathrm{R}_{1}(T ; X, Y) X, Y\right)=-\left(g_{\kappa, \tau}(Y, T)^{2}+g_{\kappa, \tau}(X, T)^{2}\right)
$$

Observamos que $\left(g_{\kappa, \tau}(Y, T)^{2}+g_{\kappa, \tau}(X, T)^{2}\right)=\|T\|^{2}=\operatorname{sen}^{2} \vartheta$, e então, substituindo os valores em (2.8), obtemos a equação desejada.

Para a equação de Codazzi, começamos com sua escrita para o caso das hipersuperfícies:

$$
\begin{aligned}
g_{\kappa, \tau}(\mathrm{R}(X, Y) Z, N)= & Y g_{\kappa, \tau}(B(X, Z), N)-g_{\kappa, \tau}\left(B\left(\nabla_{Y} X, Z\right), N\right) \\
& -g_{\kappa, \tau}\left(B\left(X, \nabla_{Y} Z\right), N\right)-X g_{\kappa, \tau}(B(Y, Z), N) \\
& +g_{\kappa, \tau}\left(B\left(\nabla_{X} Y, Z\right), N\right)+g_{\kappa, \tau}\left(B\left(Y, \nabla_{X} Z\right), N\right) .
\end{aligned}
$$

Através de cálculos diretos, podemos escrever o lado direito de (2.9) como

$$
g_{\kappa, \tau}\left(\nabla_{Y} A X-\nabla_{X} A Y+A[X, Y], Z\right) .
$$

O lado esquerdo de (2.9), pode ser escrito usando a antissimetria nas duas últimas entradas do tensor de curvatura e a Proposição 2.3, resultando em

$$
-g_{\kappa, \tau}\left(\left(\kappa-4 \tau^{2}\right) \cos \vartheta\left(g_{\kappa, \tau}(Y, T) X-g_{\kappa, \tau}(X, T) Y, Z\right)\right) .
$$

O resultado segue então ao notarmos que as expressões são válidas para qualquer $Z \in T \mathcal{M}$.

Proposição 2.5 ( [18]). Para um qualquer campo X tangente a $\mathcal{M} \subset \mathbb{E}$, vale

$$
\nabla_{X} T=\cos \vartheta(A X-\tau J X), \quad g_{\kappa, \tau}(A X-\tau J X, T)=-X(\cos \vartheta)
$$

sendo $J Y:=N \wedge Y$ para qualquer campo $Y \in T \mathcal{M}$.

Demonstração. Por um lado, temos

$$
\begin{aligned}
\bar{\nabla}_{X} E_{1} & =\bar{\nabla}_{X}(T+\cos \vartheta N) \\
& =\bar{\nabla}_{X} T+X(\cos \vartheta) N+\cos \vartheta \bar{\nabla}_{X} N \\
& =\nabla_{X} T+g_{\kappa, \tau}(A X, T) N+X(\cos \vartheta) N-\cos \vartheta A X .
\end{aligned}
$$


Por outro lado, escrevendo $X=x_{1} E_{1}+x_{2} E_{2}+x_{3} E_{3}$ e usando (2.3), temos

$$
\begin{aligned}
\bar{\nabla}_{X} E_{1} & =x_{1} \bar{\nabla}_{E_{1}} E_{1}+x_{2} \bar{\nabla}_{E_{2}} E_{1}+x_{3} \bar{\nabla}_{E_{3}} E_{1} \\
& =\tau\left(x_{3} E_{2}-x_{2} E_{3}\right) \\
& =\tau X \wedge E_{1} \\
& =\tau X \wedge(T+\cos \vartheta N) \\
& =\tau\left(g_{\kappa, \tau}(J X, T) N-\cos \vartheta J X\right) .
\end{aligned}
$$

A conclusão segue de compararmos as partes tangentes e normais das duas expressões.

Observação 2.1. Ressaltamos que, embora as proposições acima deem conta de BCV-espaços com grupo de isometria 4-dimensional, estes resultados se mantêm válidos para aqueles com grupo de isometria de dimensão 6. De fato, as demonstrações aqui expostas servem perfeitamente para o caso $\kappa=4 \tau^{2}$. Desta forma, utilizaremos estes resultados para qualquer BCV-espaço $\mathcal{N}_{\kappa, \tau}$.

Observe que $\{T, J T\}$ definidos como acima formam uma base para $T M$. Utilizando a Proposição 2.5 e supondo $\vartheta \in(0, \pi / 2]$, podemos descrever a matriz do operador de forma nesta base com a expressão

$$
A=\left(\begin{array}{cc}
\frac{T(\vartheta)}{\operatorname{sen} \vartheta} & \frac{J T(\vartheta)}{\operatorname{sen} \vartheta}-\tau \\
\frac{J T(\vartheta)}{\operatorname{sen} \vartheta}-\tau & \lambda
\end{array}\right),
$$

onde

$$
\lambda:=\frac{g_{\kappa, \tau}(A J T, J T)}{\operatorname{sen}^{2} \vartheta} .
$$

Já as componentes da conexão, que também seguem da Proposição 2.5, ficam

$$
\begin{aligned}
& \nabla_{T} T=\cot \vartheta[T(\vartheta) T+(J T(\vartheta)-2 \tau \tan \vartheta) J T], \\
& \nabla_{J T} T=\cot \vartheta J T(\vartheta) T+\lambda \cos \vartheta J T, \\
& \nabla_{T} J T=\cot \vartheta[(-J T(\vartheta)+2 \tau \tan \vartheta) T+T(\vartheta) J T], \\
& \nabla_{J T} J T=-\lambda \cos \vartheta T+\cot \vartheta J T(\vartheta) J T .
\end{aligned}
$$


Para a realização de cálculos futuros, nos será de útil considerar a ortonormalização de $\{T, J T\}$, dada por

$$
e_{1}=\frac{T}{\operatorname{sen} \vartheta}, \quad e_{2}=\frac{J T}{\operatorname{sen} \vartheta} .
$$

Nesta nova base, a matriz do operador de forma pode ser reescrita como

$$
A=\left(\begin{array}{cc}
e_{1}(\vartheta) & e_{2}(\vartheta)-\tau \\
e_{2}(\vartheta)-\tau & \lambda
\end{array}\right),
$$

e as componentes da conexão de Levi-Civita ficam

$$
\begin{array}{ll}
\nabla_{e_{1}} e_{1}=\cot \vartheta\left(e_{2}(\vartheta)-2 \tau\right) e_{2}, & \nabla_{e_{2}} e_{1}=\lambda \cot \vartheta e_{2}, \\
\nabla_{e_{1}} e_{2}=-\cot \vartheta\left(e_{2}(\vartheta)-2 \tau\right) e_{1}, & \nabla_{e_{2}} e_{2}=-\lambda \cot \vartheta e_{1} .
\end{array}
$$

Utilizando as expressões do operador de forma e das componentes da conexão, reescrevemos as equações de Gauss e Codazzi de acordo com a proposição que segue.

Proposição 2.6. Seja $\mathcal{M}$ uma superfície de um BCV-espaço $\mathcal{N}_{\kappa, \tau}$ tal que $\vartheta \in(0, \pi / 2]$. Então, as equações de Gauss e de Codazzi são equivalentes ao sistema

$$
\left\{\begin{array}{l}
e_{1} e_{2}(\vartheta)+\lambda \cot \vartheta e_{2}(\vartheta)+\cot \vartheta e_{1}(\vartheta)\left(e_{2}(\vartheta)-2 \tau\right)-e_{2} e_{1}(\vartheta)=0 \\
2 \cot \vartheta\left[\left(e_{2}(\vartheta)\right)^{2}-3 \tau e_{2}(\vartheta)+2 \tau^{2}\right]+e_{1}(\lambda)+\lambda^{2} \cot \vartheta \\
-\lambda \cot \vartheta e_{1}(\vartheta)-e_{2} e_{2}(\vartheta)-\left(4 \tau^{2}-\kappa\right) \cos \vartheta \operatorname{sen} \vartheta=0
\end{array}\right.
$$

Demonstração. A equação de Gauss (2.6) é dada por

$$
K=g_{\kappa, \tau}\left(\nabla_{e_{2}} \nabla_{e_{1}} e_{1}-\nabla_{e_{1}} \nabla_{e_{2}} e_{1}+\nabla_{\left[e_{1}, e_{2}\right]} e_{1}, e_{2}\right)
$$

onde

$$
\begin{aligned}
& \left.\left.\nabla_{e_{2}} \nabla_{e_{1}} e_{1}=e_{2}\left[\cot \vartheta\left(e_{2}(\vartheta)-2 \tau\right)\right)\right] e_{2}-\left[\cot \vartheta\left(e_{2}(\vartheta)-2 \tau\right)\right)\right](\lambda \cot \vartheta) e_{1} \\
& \nabla_{e_{1}} \nabla_{e_{2}} e_{1}=e_{1}(\lambda \cot \vartheta) e_{2}-(\lambda \cot \vartheta) \cot \vartheta\left(e_{2}(\vartheta)-2 \tau\right) e_{1} \\
& \nabla_{\left[e_{1}, e_{2}\right]} e_{1}=-\cot ^{2} \vartheta\left(\left(e_{2}(\vartheta)-2 \tau\right)^{2}+\lambda^{2}\right) e_{2} .
\end{aligned}
$$


Além disso, temos

$$
\operatorname{det} A=\lambda e_{1}(\vartheta)-\left(e_{2}(\vartheta)-\tau\right)^{2}
$$

e, logo, a expressão para a equação de Gauss na base $\left\{e_{1}, e_{2}\right\}$ é

$$
\begin{aligned}
& \tan \vartheta\left[e_{2} e_{2}(\vartheta)-e_{1}(\lambda)\right]+\lambda e_{1}(\vartheta)-\lambda^{2}-2\left(e_{2}(\vartheta)\right)^{2} \\
& -4 \tau^{2} \cos ^{2} \vartheta+6 \tau e_{2}(\vartheta)-\kappa \operatorname{sen}^{2} \vartheta=0 .
\end{aligned}
$$

Para a equação de Codazzi, fazemos $X=e_{1}$ e $Y=e_{2}$ em (2.7), obtendo

$$
\nabla_{e_{1}} A\left(e_{2}\right)-\nabla_{e_{2}} A\left(e_{1}\right)-A\left(\left[e_{1}, e_{2}\right]\right)=\left(4 \tau^{2}-\kappa\right) \cos \vartheta \operatorname{sen} \vartheta e_{2}
$$

onde,

$$
\begin{aligned}
\nabla_{e_{1}} A\left(e_{2}\right) & =\left[e_{1}\left(e_{2}(\vartheta)-\tau\right)-\lambda \cot \vartheta\left(e_{2}(\vartheta)-2 \tau\right)\right] e_{1}+\left[\cot \vartheta\left(e_{2}(\vartheta)-\tau\right)\left(e_{2}(\vartheta)-2 \tau\right)\right] e_{2} \\
\nabla_{e_{2}} A\left(e_{1}\right) & =\left[e_{2} e_{1}(\vartheta)-\lambda \cot \vartheta\left(e_{2}(\vartheta)-\tau\right)\right] e_{1}+\left[\lambda \cot \vartheta e_{1}(\vartheta)+e_{2}\left(e_{2}(\vartheta)-\tau\right)\right] e_{2} \\
A\left(\left[e_{1}, e_{2}\right]\right) & =\left[\cot \vartheta\left(-\left(e_{2}(\vartheta)-2 \tau\right) e_{1}(\vartheta)-\lambda\left(e_{2}(\vartheta)-\tau\right)\right)\right] e_{1} \\
& +\left[\cot \vartheta\left(-\left(e_{2}(\vartheta)-2 \tau\right)\left(e_{2}(\vartheta)-\tau\right)-\lambda^{2}\right)\right] e_{2}
\end{aligned}
$$

Desta forma, a equação de Codazzi é equivalente às condições:

$$
\left\{\begin{array}{l}
e_{1} e_{2}(\vartheta)+\lambda \cot \vartheta e_{2}(\vartheta)+\cot \vartheta e_{1}(\vartheta)\left(e_{2}(\vartheta)-2 \tau\right)-e_{2} e_{1}(\vartheta)=0, \\
2 \cot \vartheta\left[\left(e_{2}(\vartheta)\right)^{2}-3 \tau e_{2}(\vartheta)+2 \tau^{2}\right]+e_{1}(\lambda)+\lambda^{2} \cot \vartheta \\
-\lambda \cot \vartheta e_{1}(\vartheta)-e_{2} e_{2}(\vartheta)-\left(4 \tau^{2}-\kappa\right) \cos \vartheta \operatorname{sen} \vartheta=0 .
\end{array}\right.
$$

Observamos que a segunda equação deste sistema garante que a equação de Gauss seja satisfeita e, assim, concluímos a demonstração.

\subsection{O problema de biconservatividade}

Como descrito na Seção 1.1 os conceitos de aplicação harmônica e bi-harmônica têm sua origem em problemas variacionais. Em [36] Hilbert mostra que a cada problema variacional pode 
ser associado um tensor energia-momento $S$, o qual é 2-covariante e simétrico nos pontos críticos, i.e., $\operatorname{div} S=0$. No caso de uma aplicação harmônica entre variedades Riemannianas, $\phi:(\mathcal{M}, g) \rightarrow(\mathcal{N}, h)$, este tensor tem a expressão

$$
S=\frac{1}{2}\|d \phi\|_{h}^{2} g-\phi^{*} h
$$

sendo estudado em detalhes por Baird e Eells em [3], e por Sanini em [66]. Ressaltamos, que $S$ assim definido satisfaz $\operatorname{div} S=-h(\tau(\phi), d \phi), \log$ o div $S=0$ para as aplicações harmônicas.

Observe que se $\phi$ for uma imersão isométrica, valerá sempre $\operatorname{div} S=0$, já que $\tau(\phi)$ será normal à variedade imersa.

Para as aplicações bi-harmônicas, o tensor energia-momento foi obtido por Jiang em [41] e a sua expressão é dada por

$$
\begin{aligned}
S_{2}(X, Y)= & \frac{1}{2}\|\tau(\phi)\|_{h}^{2} g(X, Y)+h(d \phi, \nabla \tau(\phi)) g(X, Y) \\
& -h\left(d \phi(X), \nabla_{Y} \tau(\phi)\right)-h\left(d \phi(Y), \nabla_{X} \tau(\phi)\right), \quad X, Y \in T \mathcal{M}
\end{aligned}
$$

Posteriormente $S_{2}$ foi estudado em [45], onde os autores obtiveram exemplos de aplicações biharmônicas. O tensor $S_{2}$ assim definido satisfaz

$$
\operatorname{div} S_{2}=-\left\langle\tau_{2}(\phi), d \phi\right\rangle
$$

confirmando o princípio de tensor energia-momento para o funcional bienergia, uma vez que $\tau_{2}(\phi)=0$ se, e somente se, $\phi$ for um ponto crítico deste funcional.

Ao contrário do que ocorre no caso harmônico, o fato de $\phi$ ser uma imersão isométrica não implica necessariamente que o divergente de $S_{2}$ seja nulo. Ao invés disso, temos

$$
\operatorname{div} S_{2}=-h\left(\tau_{2}(\phi), d \phi\right)
$$

onde $\tau_{2}(\phi)^{T}$ representa a parte tangente do campo de bitensão dado em (1.1). Portanto, a condição $\operatorname{div} S_{2}=0$ equivale a $\tau_{2}(\phi)^{T}=0$. 
No estudo de imersões bi-harmônicas, uma das técnicas largamente utilizada consiste na decomposição do campo de bitensão em sua parte normal e tangente e, então, verificam-se as condições obtidas ao impor que cada uma dessas partes se anule. Tal decomposição do campo de bitensão pode ser encontrada, por exemplo, em [5, 17, 44, 57, 59]. Para o caso de hipersuperfícies, temos o seguinte teorema:

Teorema 2.7 ( [57]). Seja $\phi: \mathcal{M}^{n-1} \rightarrow \mathcal{N}^{n}$ uma imersão isométrica com função curvatura média $f$ e vetor curvatura média $H=(f /(n-1)) N$. Então, $\phi$ será bi-harmônica se, e somente se, as componentes normal e tangente de $\tau_{2}(\phi)$ se anularem, i.e. respectivamente

$$
\Delta f+f\|A\|^{2}-f \operatorname{Ricci}^{\mathcal{N}}(N, N)=0
$$

$e$

$$
2 A(\operatorname{grad} f)+f \operatorname{grad} f-2 f \operatorname{Ricci}^{\mathcal{N}}(N)^{T}=0,
$$

sendo A o operador de forma e $\operatorname{Ricci}(N)^{T}$ a componente tangente da curvatura de Ricci de $\mathcal{N}$ na direção do campo normal unitário $N$ de $\mathcal{M}$ em $\mathcal{N}$.

Assim fica claro que, para que uma hipersuperfície $\mathcal{M}$, como no Teorema 2.7, satisfaça

$$
\operatorname{div} S_{2}=0
$$

é necessário e suficiente que (2.14) seja satisfeita. Dito isto, podemos apresentar o conceito de hipersuperfícies biconservativas, que está claramente relacionado ao conceito de aplicações biharmônicas. Temos a seguinte

Definição 2.8. Uma imersão isométrica $\phi: \mathcal{M}^{n-1} \rightarrow \mathcal{N}^{n}$ com vetor curvatura média $H=$ $(f /(n-1)) N$ será dita biconservativa se $\phi$ satisfizer a condição (2.14). A hipersuperfície $\mathcal{M}^{n-1}$ assim imersa também será chamada de biconservativa.

Como toda hipersuperfície bi-harmônica é biconservativa, as que não são bi-harmônicas são chamadas de biconservativas próprias. 
O conceito de hipersuperfícies biconservativas foi introduzido por Caddeo, Montaldo, Oniciuc e Piu em [10], onde os autores classificam localmente estas hipersuperfícies nos espaços forma. Já em [50], são estudadas hipersuperfícies biconservativas próprias do espaço Euclidiano $\mathbb{R}^{n}$ que são invariantes pela ação do grupo $S O(p+1) \times S O(q+1)$, no caso em que $n=p+q+2$, e pela ação do grupo $S O(p+1)$ no caso em que $n=p+2$; utilizando esse estudo os autores obtêm exemplos de imersões bi-harmônicas próprias. Em [68] são consideradas as superfícies biconservativas do tipo espaço e do tipo tempo no espaço de Minkowski tridimensional, sendo dada a parametrização para aquelas que não são de curvatura média constante. Ainda, em [34] são classificadas as superfícies biconservativas não mínimas que possuem vetor curvatura média paralelo nos espaços produtos $\mathbb{S}^{n} \times \mathbb{R}$ e $\mathbb{H}^{n} \times \mathbb{R}$; além disso, os autores consideram as superfícies biconservativas de curvatura média constante em espaços de Hadamard obtendo resultados sobre sua compacidade.

Este capítulo é dedicado ao estudo das superfícies biconservativas nos BCV-espaços. Mais especificamente, consideramos as superfícies biconservativas que fazem ângulo constante com o campo de Hopf (ver Seção 2.2), aquelas que possuem curvatura média constante (CMC) e as de rotação ao redor do campo de Hopf.

\subsection{Superfícies biconservativas com ângulo constante em BCV-espaços}

Nesta seção estudamos as superfícies biconservativas de ângulo constante nos BCV-espaços, obtendo na Proposição 2.11 que estas superfícies são CMC e, por fim, mostramos que as superfícies biconservativas $\mathrm{CMC}$ dos $\mathrm{BCV}$-espaços que não são espaços forma devem ser cilindros de Hopf sobre curvas de curvatura geodésica constante. Ao longo desta seção adotaremos a notação da Seção 2.2. desta forma, temos a definição que segue:

Definição 2.9. Dizemos que uma superfície de um BCV-espaço $\mathcal{N}_{\kappa, \tau}$ é uma superfície do tipo hélice ou uma superfície de ângulo constante se for orientável e o ângulo $\vartheta \in[0, \pi)$ entre seu 
campo de vetores normal unitário e o campo de Killing unitário $E_{1}$ (tangente à fibração de Hopf) é constante para todos os ponto da superfície.

Começamos verificando quais condições são obtidas ao impormos que uma superfície qualquer em um BCV-espaço seja biconservativa.

Proposição 2.10. Seja $\mathcal{M} \subset \mathcal{N}_{\kappa, \tau}$ uma superfície em um $B C V$-espaço com $\vartheta \in(0, \pi / 2]$. Para que $\mathcal{M}$ seja biconservativa, é necessário e suficiente que

$$
\left\{\begin{array}{l}
e_{1}\left(\lambda+e_{1}(\vartheta)\right)\left(\lambda+3 e_{1}(\vartheta)\right)+2 e_{2}\left(\lambda+e_{1}(\vartheta)\right)\left(e_{2}(\vartheta)-\tau\right) \\
-2\left(4 \tau^{2}-\kappa\right)\left(\lambda+e_{1}(\vartheta)\right) \cos \vartheta \operatorname{sen} \vartheta=0, \\
2 e_{1}\left(\lambda+e_{1}(\vartheta)\right)\left(e_{2}(\vartheta)-\tau\right)+\left(3 \lambda+e_{1}(\vartheta)\right) e_{2}\left(\lambda+e_{1}(\vartheta)\right)=0,
\end{array}\right.
$$

onde $\left\{e_{1}, e_{2}\right\}$ são os campos de vetores tangentes a $\mathcal{M}$ dados em (2.11.

Demonstração. Pondo $e_{1}=\sum a_{i} E_{i}, e_{2}=\sum b_{i} E_{i}$ e $N=\sum c_{i} E_{i}$ e utilizando (2.5), obtemos

$$
\begin{aligned}
\operatorname{Ricci}(N)^{T} & =\operatorname{Ricci}\left(N, e_{1}\right) e_{1}+\operatorname{Ricci}\left(N, e_{2}\right) e_{2} \\
& =\left[\left(\kappa-2 \tau^{2}\right)\left(c_{2} a_{2}+c_{3} a_{3}\right)+2 \tau^{2} c_{1} a_{1}\right] e_{1} \\
& +\left[\left(\kappa-2 \tau^{2}\right)\left(c_{2} b_{2}+c_{3} b_{3}\right)+2 \tau^{2} c_{1} b_{1}\right] e_{2} \\
& =\left(4 \tau^{2}-\kappa\right) c_{1}\left(a_{1} e_{1}+b_{1} e_{2}\right) .
\end{aligned}
$$

Pela construção de $\left\{e_{1}, e_{2}\right\}$, temos que $a_{1}=\operatorname{sen} \vartheta, b_{1}=0$ e $c_{3}=\cos \vartheta$, portanto

$$
\operatorname{Ricci}(N)^{T}=\left(4 \tau^{2}-\kappa\right) \cos \vartheta \operatorname{sen} \vartheta e_{1} .
$$

De (2.12), segue que a função curvatura média

$$
f=\lambda+e_{1}(\vartheta)
$$

$\log 0$

$$
\operatorname{grad} f=\left(e_{1}(\lambda)+e_{1} e_{1}(\vartheta)\right) e_{1}+\left(e_{2}(\lambda)+e_{2} e_{1}(\vartheta)\right) e_{2} .
$$


Usando (2.12) temos

$$
\begin{aligned}
A(\operatorname{grad} f) & =\left[\left(e_{1}(\lambda)+e_{1} e_{1}(\vartheta)\right) e_{1}(\vartheta)+\left(e_{2}(\lambda)+e_{2} e_{1}(\vartheta)\right)\left(e_{2}(\vartheta)-\tau\right)\right] e_{1} \\
& +\left[\left(e_{1}(\lambda)+e_{1} e_{1}(\vartheta)\right)\left(e_{2}(\vartheta)-\tau\right)+\left(e_{2}(\lambda)+e_{2} e_{1}(\vartheta)\right) \lambda\right] e_{2} .
\end{aligned}
$$

Dessa forma, juntando (2.16), (2.17) e (2.18), concluímos que a equação de biconservatividade é equivalente ao sistema (2.15).

Agora nos encontramos em posição de estudar o caso de superfícies de ângulo constante.

Proposição 2.11. Seja $\mathcal{M}$ uma superfície biconservativa própria de um $B C V$-espaço $\mathcal{N}_{\kappa, \tau}$. Se $\mathcal{M}$ for de ângulo constante $\vartheta \in[0, \pi / 2]$, então possuirá curvatura média constante.

Demonstração. Primeiro consideramos o caso em que $\vartheta \in(0, \pi / 2)$. Neste caso a matriz do operador de forma na base $\left\{e_{1}, e_{2}\right\}$ é dada por

$$
A=\left(\begin{array}{cc}
0 & -\tau \\
-\tau & \lambda
\end{array}\right)
$$

$\log 0$

$$
f=\lambda \text {. }
$$

A primeira das equações de Codazzi (2.13) é automaticamente satisfeita, enquanto a segunda se reduz a

$$
e_{1}(\lambda)+\lambda^{2} \cot \vartheta+\kappa \cos \vartheta \operatorname{sen} \vartheta+4 \tau^{2} \cot \vartheta \cos ^{2} \vartheta=0
$$

Já as de biconservatividade (2.15) nos dão o sistema

$$
\left\{\begin{array}{l}
\lambda e_{1}(\lambda)-2 \tau e_{2}(\lambda)-2 \lambda \cos \vartheta \operatorname{sen} \vartheta\left(4 \tau^{2}-\kappa\right)=0 \\
3 \lambda e_{2}(\lambda)=2 \tau e_{1}(\lambda)
\end{array}\right.
$$


Agora, usando a segunda equação de (2.20) na primeira, e tendo em conta (2.19) obtemos o seguinte polinômio em $\lambda$, com coeficientes constantes:

$$
\begin{aligned}
& \cot \vartheta \lambda^{4}+\left[\cos \vartheta \operatorname{sen} \vartheta\left(8 \tau^{2}-\kappa\right)+4 \tau^{2} \cot \vartheta\left(\cos ^{2} \vartheta-\frac{1}{3}\right)\right] \lambda^{2} \\
& -\frac{4}{3} \tau^{2} \cos \vartheta\left(\kappa \operatorname{sen} \vartheta+4 \tau^{2} \cot \vartheta \cos \vartheta\right)=0 .
\end{aligned}
$$

Portanto, $f$ é constante, ou seja a superfície é CMC.

Para o caso em que $\vartheta=\pi / 2$, o sistema (2.15) se torna

$$
\left\{\begin{array}{l}
\lambda e_{1}(\lambda)=2 \tau e_{2}(\lambda) \\
3 \lambda e_{2}(\lambda)=2 \tau e_{1}(\lambda)
\end{array}\right.
$$

donde segue, de imediato, que $\lambda$ deve ser constante.

Já no caso em que $\vartheta=0$, ocorre $\operatorname{sen} \vartheta=0$ e, então, $E_{2}$ e $E_{3}$, definidos em (2.2), devem ser tangentes à superfície. Desta forma, a distribuição determinada por $\left\{E_{2}, E_{3}\right\}$ deve ser integrável e, pelo Teorema de Frobenius, deve ser involutiva. Segue que $\tau=0$ e, portanto, a superfície deve ser $\mathbb{H}^{2} \times\{p\} \subset \mathbb{H}^{2} \times \mathbb{R}, \mathbb{S}^{2} \times\{p\} \subset \mathbb{S}^{2} \times \mathbb{R}$ ou $\mathbb{R}^{2} \times\{p\} \subset \mathbb{R}^{3}$, onde $p \in \mathbb{R}$. Estas superfícies são harmônicas e portanto não são biconservativas próprias.

Proposição 2.12. Seja $\mathcal{M}$ uma superfície biconservativa própria de um BCV-espaço $\mathcal{N}_{\kappa, \tau}$ que não seja um espaço forma (i.e. $\kappa \neq 4 \tau^{2}$ ). Se $\mathcal{M}$ possuir curvatura média constante, então será um cilindro de Hopf sobre uma curva que possui curvatura geodésica constante.

Demonstração. Ao considerarmos superfícies biconservativas de curvatura média constante, a equação (2.14) nos dá

$$
f \operatorname{Ricci}(N)^{T}=0 \text {. }
$$

Então, descartando o caso harmônico, e usando (2.16) obtemos a condição

$$
\left(4 \tau^{2}-\kappa\right) \cos \vartheta \operatorname{sen} \vartheta=0
$$

Concluímos que uma das seguintes possibilidades deve ocorrer: 
1) $4 \tau^{2}-\kappa=0$, caso em que o BCV-espaço é uma forma espacial;

2) $\operatorname{sen} \vartheta=0$. Como vimos na demonstração da Proposição 2.11 este caso gera superfícies harmônicas;

3) $\cos \vartheta=0$.

Descartados os primeiro e segundo casos pelas hipóteses da proposição, vejamos mais a fundo o terceiro caso. No que segue, usamos ideias presentes em [57, 58]. Como consequência imediata de $\cos \vartheta=0$, temos $e_{1}=E_{1}$. Segue do fato de as curvas integrais de $e_{1}$ serem geodésicas de $\mathcal{M}$ que podemos parametrizar a superfície por $\mathbf{x}=\mathbf{x}(u, v)$, de forma que as $u$-curvas sejam as curvas integrais de $e_{1}$ e as $v$-curvas sejam ortogonais às $u$-curvas.

Consideramos então a submersão de Hopf

$$
\psi: \mathcal{N}_{\kappa, \tau} \rightarrow M^{2}(\kappa)=\left(\mathbb{R}^{2}, h=\frac{d x^{2}+d y^{2}}{\left[1+\frac{\kappa}{4}\left(x^{2}+y^{2}\right)\right]^{2}}\right), \quad \psi(x, y, z)=(x, y)
$$

e também

$$
\begin{aligned}
\beta: I & \longrightarrow \mathcal{N}_{\kappa, \tau} \\
s & \longmapsto \beta(s)
\end{aligned}
$$

uma $v$-curva parametrizada por comprimento de arco. Desta forma, $\beta$ será horizontal à submersão $\psi$. Seja

$$
\alpha(s)=\psi(\beta(s))
$$

a curva no espaço base da submersão, gerada pela projeção de $\beta$, então podemos ver a superfície $\mathcal{M}$ (localmente) como união de pré-imagens de $\alpha$ :

$$
\mathcal{M}=\cup_{s \in I} \psi^{-1}(\alpha(s))
$$

Segue que a superfície $\mathcal{M}$ é um cilindro de Hopf sobre a curva $\alpha(s)$. 
Considere o referencial de Frenet de $\alpha$ em $M^{2}(\kappa)$ dado por $\left\{\mathbf{t}=\alpha^{\prime}, \mathbf{n}\right\}$, e $\kappa_{g}$ sua curvatura geodésica. Das fórmulas de Frenet temos

$$
\begin{aligned}
& \widehat{\nabla}_{\mathbf{t}} \mathbf{t}=\kappa_{g} \mathbf{n}, \\
& \widehat{\nabla}_{\mathbf{t}} \mathbf{n}=-\kappa_{g} \mathbf{t},
\end{aligned}
$$

onde $\widehat{\nabla}$ denota a conexão em $M^{2}(\kappa)$. Dessa forma, sendo $\psi$ uma submersão Riemanniana,

$$
\alpha^{\prime}=\mathrm{d} \psi\left(e_{2}\right), \quad \mathbf{n}=\mathrm{d} \psi(N),
$$

$e_{2}$ e $N$ campos horizontais à submersão, resulta que

$$
\kappa_{g}=h\left(\widehat{\nabla}_{\mathbf{t}} \mathbf{t}, \mathbf{n}\right)=g_{\kappa, \tau}\left(\bar{\nabla}_{e_{2}} e_{2}, N\right)=f .
$$

Concluímos assim que $\kappa_{g}$ é constante, terminando a demonstração.

Como consequência direta dos resultados supracitados, podemos enunciar o seguinte teorema.

Teorema 2.13. Seja $\mathcal{M}$ uma superfície biconservativa própria de um BCV-espaço $\mathcal{N}_{\kappa, \tau}$ que não seja um espaço forma (i.e. $\left.\kappa \neq 4 \tau^{2}\right)$. Então as seguintes afirmações são equivalentes:

1) $\mathcal{M}$ é uma superfície de ângulo constante;

2) $\mathcal{M}$ é uma superfície $C M C$;

3) $\mathcal{M}$ é um cilindro de Hopf sobre uma curva com curvatura geodésica constante.

\subsection{Superfícies de revolução biconservativas}

Nesta seção trataremos de superfícies invariantes pela ação de um subgrupo do grupo de isometrias do espaço ambiente. Sendo assim, relembramos a seguir algumas propriedades da teoria de geometria equivariante.

Seja $(\mathcal{N}, g)$ uma variedade Riemanniana e $G$ um subgrupo fechado do grupo de isometria $\mathfrak{I s o}(\mathcal{N}, g)$. Para $x \in \mathcal{N}$, denotaremos por $a x$ a ação do elemento $a \in G$ em $x$ e por: 
- $G(x):=\{a x: a \in G\}$, a órbita de $x$

- $G_{x}:=\{a \in G: a x=x\}$, o grupo de isotropia de $x$;

- $\mathcal{B}=\mathcal{N} / G$, o espaço das órbitas.

Da teoria de ações Riemannianas sabemos que:

- Existe uma única classe minimal de conjugação de subgrupos de isotropia. As órbitas com isotropia nesta classe são chamadas de principais. A união das órbitas principais, denotada por $\mathcal{N}_{r}$, é chamada de parte regular de $\mathcal{N}$. O conjunto $\mathcal{N}_{r}$ é aberto e denso em $\mathcal{N}$.

- O grupo de isotropia $G_{x}$ é compacto e $G / G_{x}$ é difeomorfo a $G(x)$. Todas as órbitas com isotropia na mesma classe de conjugação são duas a duas difeomorfas. Em particular, todas as órbitas principais são duas a duas difeomorfas.

- A parte regular do espaço das órbitas $\mathcal{B}_{r}:=\mathcal{N}_{r} / G$ é uma variedade diferenciável conexa (se $\mathcal{N}$ é conexo) e a aplicação quociente $\pi: \mathcal{N}_{r} \rightarrow \mathcal{B}_{r}$ é uma submersão.

Observação 2.2. $O$ espaço das órbitas $\mathcal{B}$ pode conter singularidades devido a presença de órbitas não principais. Porém, para o caso em que as órbitas principais possuem codimensão menor ou igual a 2 (caso no qual se enquadra nosso estudo), o espaço das órbitas é sempre uma variedade diferenciável, com ou sem bordo.

Na sequência assumiremos que $(\mathcal{N}, g)$ seja uma variedade Riemanniana tridimensional. Dado $X$ um campo de Killing de $\mathcal{N}$, temos que $X$ gera um subgrupo a um parâmetro $G_{X}$ do grupo de isometrias $\mathfrak{I s o}(\mathcal{N}, g)$. Seja $\phi: \mathcal{M}^{2} \rightarrow \mathcal{N}^{3}$ uma imersão de $\mathcal{M}$ em $\mathcal{N}$. Dizemos que $\phi$ é uma imersão $G_{X}$-equivariante, e $\phi(\mathcal{M})$ uma superfície $G_{X}$-equivariante de $\mathcal{N}$, se existir uma ação de $G_{X}$ sobre $\mathcal{M}$ tal que para $x \in \mathcal{M}$ e $a \in G_{X}$ tenhamos $\phi(a x)=a \phi(x)$. Equiparemos $\mathcal{M}$ com a métrica induzida pela imersão $\phi$ e assumiremos que $\phi(\mathcal{M}) \subset \mathcal{N}_{r}$ e que $\mathcal{N} / G_{X}$ é conexo. Dessa forma, $\phi$ induz uma imersão $\widetilde{\phi}: \mathcal{M} / G_{X} \rightarrow \mathcal{N}_{r} / G_{X}$ entre os espaços das órbitas; além disso, o 
espaço $\mathcal{N}_{r} / G_{X}$ pode se equipado com uma métrica Riemanniana, a métrica quociente, de forma que a aplicação quociente $\pi: \mathcal{N}_{r} \rightarrow \mathcal{N}_{r} / G_{X}$ seja uma submersão Riemanniana. Observe que $\mathcal{N}_{r} / G_{X}$ é uma superfície e $\widetilde{\phi}$ define uma curva em $\mathcal{N}_{r} / G_{X}$ chamada de curva geradora.

É sabido que $\mathcal{N}_{r} / G_{X}$ pode ser localmente parametrizado por funções invariantes do campo de Killing $X$ (ver, por exemplo, [55]). Se $\left\{\zeta_{1}, \zeta_{2}\right\}$ é um conjunto completo de funções invariantes que parametrizam um subconjunto aberto e $G_{X}$-invariante de $\mathcal{N}_{r}$, então a métrica quociente é dada por

$$
\widetilde{g}=\sum_{i, j=1}^{2} h^{i j} \mathrm{~d} \zeta_{i} \otimes \mathrm{d} \zeta_{j},
$$

onde $\left(h^{i j}\right)$ representa inversa da matriz $\left(h_{i j}\right)$ cujas entradas são

$$
h_{i j}=g\left(\operatorname{grad} \zeta_{i}, \operatorname{grad} \zeta_{j}\right)
$$

sendo grad o gradiente de $(\mathcal{N}, g)$.

A função curvatura média da superfície invariante imersa se relaciona com a curvatura geodésica da curva da forma descrita no teorema que segue.

Teorema 2.14 ( [2]]). Seja f a função curvatura média de $\mathcal{M}_{r} \subset \mathcal{N}_{r}$ e $k_{g}$ a curvatura geodésica da curva geradora $\mathcal{M}_{r} / G_{X} \subset \mathcal{B}_{r}$ com respeito a métrica quociente $\widetilde{g}$. Então

$$
f(x)=k_{g}(\pi(x))-D_{\mathbf{n}} \log \omega(\pi(x)), \quad x \in \mathcal{M}_{r},
$$

onde $\mathbf{n}$ é o campo de vetores unitário normal à curva geradora e $\omega=\sqrt{g(X, X)}$ é a função volume da órbita principal $\pi(x)$.

\subsubsection{Superfícies de revolução biconservativas em BCV-espaços}

Consideraremos, a partir de agora, as superfícies de um BCV-espaço $\mathcal{N}_{\kappa, \tau}$, que sejam invariantes pelo grupo de isometrias $G_{X}$ gerado pelo campo de Killing

$$
X=-y \frac{\partial}{\partial x}+x \frac{\partial}{\partial y} .
$$


Estas superfícies são ditas de revolução em torno do eixo z. Excluindo as órbitas dos pontos ao longo do eixo $z$, as órbitas geradas pela ação de $G_{X}$ são todas principais e a teoria acima exposta se aplica.

Fazendo a mudança de coordenadas:

$$
\left\{\begin{array}{l}
x=r \cos \theta \\
y=r \operatorname{sen} \theta \\
z=z
\end{array}\right.
$$

onde $r \geq 0$, temos

$$
\left\{\begin{array}{l}
d x=\cos \theta d r-r \operatorname{sen} \theta d \theta \\
d y=\operatorname{sen} \theta d r+r \cos \theta d \theta \\
d z=d z
\end{array}\right.
$$

e, sua base dual é dada por:

$$
\left\{\begin{array}{l}
\frac{\partial}{\partial x}=\cos \theta \frac{\partial}{\partial r}-\frac{\operatorname{sen} \theta}{r} \frac{\partial}{\partial \theta} \\
\frac{\partial}{\partial y}=\operatorname{sen} \theta \frac{\partial}{\partial r}+\frac{\cos \theta}{r} \frac{\partial}{\partial \theta} \\
\frac{\partial}{\partial z}=\frac{\partial}{\partial z}
\end{array}\right.
$$

Neste caso, a métrica (2.1) pode ser reescrita como

$$
g_{\kappa, \tau}=\frac{d r^{2}}{F^{2}}+r^{2}\left(\frac{1+\tau^{2} r^{2}}{F^{2}}\right) d \theta^{2}+d z^{2}-2 \frac{\tau r^{2}}{F} d \theta d z
$$

onde $F=1+\frac{\kappa}{4} r^{2}$. Portanto, a matriz associada à métrica $g_{\kappa, \tau}$ é

$$
\left(\left(g_{\kappa, \tau}\right)_{i j}\right)=\left(\begin{array}{ccc}
\frac{1}{F^{2}} & 0 & 0 \\
0 & \frac{r^{2}\left(1+\tau^{2} r^{2}\right)}{F^{2}} & -\frac{\tau r^{2}}{F} \\
0 & -\frac{\tau r^{2}}{F} & 1
\end{array}\right)
$$


cuja inversa é dada por

$$
\left(\left(g_{\kappa, \tau}\right)^{i j}\right)=\left(\begin{array}{ccc}
F^{2} & 0 & 0 \\
0 & \frac{F^{2}}{r^{2}} & \tau F \\
0 & \tau F & 1+r^{2} \tau^{2}
\end{array}\right) .
$$

Sendo que a expressão do campo de Killing $X$ nas coordenadas cilíndricas é

$$
X=\frac{\partial}{\partial \theta}
$$

as funções invariantes que parametrizam o espaço das órbitas são:

$$
u(r, \theta, z)=r, \quad v(r, \theta, z)=z .
$$

Dessa forma, temos que

$$
\mathcal{B}=\left\{(r, z) \in \mathbb{R}^{2}: r \geq 0\right\}
$$

Nosso próximo passo é calcular a métrica quociente do espaço das órbitas. Temos:

Proposição 2.15. A métrica quociente $\widetilde{g}$ para o espaço das órbitas $\mathcal{B}$ é dada por

$$
\widetilde{g}=\frac{d r^{2}}{F^{2}}+\frac{d z^{2}}{1+\tau^{2} r^{2}}
$$

Demonstração. Para determinar $\widetilde{g}$ usaremos (2.21). Como

$$
\begin{array}{ll}
(\operatorname{grad} u)_{1}=F^{2}, & (\operatorname{grad} v)_{1}=0, \\
(\operatorname{grad} u)_{2}=0, & (\operatorname{grad} v)_{2}=\tau F, \\
(\operatorname{grad} u)_{3}=0, & (\operatorname{grad} v)_{3}=\left(1+\tau^{2} r^{2}\right),
\end{array}
$$

então,

$$
\operatorname{grad} u=F^{2} \frac{\partial}{\partial r}, \quad \operatorname{grad} v=\tau F \frac{\partial}{\partial \theta}+\left(1+\tau^{2} r^{2}\right) \frac{\partial}{\partial z}
$$

Dessa forma,

$$
\begin{aligned}
& h_{11}=g_{\kappa, \tau}(\operatorname{grad} u, \operatorname{grad} u)=F^{2}, \\
& h_{12}=g_{\kappa, \tau}(\operatorname{grad} u, \operatorname{grad} v)=0=h_{21}, \\
& h_{22}=g_{\kappa, \tau}(\operatorname{grad} v, \operatorname{grad} v)=1+\tau^{2} r^{2},
\end{aligned}
$$


logo,

$$
\left(h_{i j}\right)=\left(\begin{array}{cc}
F^{2} & 0 \\
0 & \left(1+\tau^{2} r^{2}\right)
\end{array}\right) .
$$

Segue que a matriz da métrica $\widetilde{g}$ é dada por

$$
\left(\widetilde{g}_{i j}\right)=\left(\begin{array}{cc}
\frac{1}{F^{2}} & 0 \\
0 & \frac{1}{\left(1+\tau^{2} r^{2}\right)}
\end{array}\right)
$$

e, portanto, a métrica $\widetilde{g}$ deve ser como em (2.23).

Agora, considere a superfície $\mathcal{M}$ parametrizada por

$$
\Phi(\theta, s)=(r(s), \theta, z(s))
$$

a qual é obtida pela ação do grupo de isometrias $G_{X}$ sobre uma curva do espaço das órbitas

$$
\gamma(s)=(r(s), z(s))
$$

Consideramos $\gamma$ parametrizada pelo comprimento de arco em $(\mathcal{B}, \widetilde{g})$, o que nos confere

$$
\frac{r^{\prime 2}}{F^{2}}+\frac{z^{\prime 2}}{1+\tau^{2} r^{2}}=1
$$

O espaço tangente à superfície $G_{X}$-invariante $\mathcal{M}$ será gerado por

$$
\left\{\begin{array}{l}
\Phi_{\theta}=(0,1,0)=\frac{\partial}{\partial \theta} \\
\Phi_{s}=\left(r^{\prime}, 0, z^{\prime}\right)=r^{\prime} \frac{\partial}{\partial r}+z^{\prime} \frac{\partial}{\partial z} .
\end{array}\right.
$$

Usando (2.2) e (2.22) podemos escrever

$$
\left\{\begin{array}{l}
\frac{\partial}{\partial r}=\frac{\cos \theta}{F} E_{2}+\frac{\operatorname{sen} \theta}{F} E_{3} \\
\frac{\partial}{\partial \theta}=-\frac{r^{2} \tau}{F} E_{1}-\frac{r \operatorname{sen} \theta}{F} E_{2}+\frac{r \cos \theta}{F} E_{3} \\
\frac{\partial}{\partial z}=E_{1}
\end{array}\right.
$$


e, então, obtemos $X_{\theta}$ e $X_{s}$ na base ortonormal $\left\{E_{1}, E_{2}, E_{3}\right\}$ :

$$
\left\{\begin{array}{l}
\Phi_{\theta}=-\frac{r^{2} \tau}{F} E_{1}-\frac{r \operatorname{sen} \theta}{F} E_{2}+\frac{r \cos \theta}{F} E_{3}, \\
\Phi_{s}=z^{\prime} E_{1}+r^{\prime}\left(\frac{\cos \theta}{F} E_{2}+\frac{\operatorname{sen} \theta}{F} E_{3}\right) .
\end{array}\right.
$$

Usando (2.25), calculamos a expressão do campo de vetores normal unitário

$$
\begin{aligned}
N & =\frac{\Phi_{s} \wedge \Phi_{\theta}}{\left\|\Phi_{s} \wedge \Phi_{\theta}\right\|_{g_{\kappa, \tau}}} \\
& =\frac{r^{\prime}}{F \sqrt{1+\tau^{2} r^{2}}} E_{1}+\frac{\left(-z^{\prime} \cos \theta-\frac{r r^{\prime} \tau \operatorname{sen} \theta}{F}\right)}{\sqrt{1+\tau^{2} r^{2}}} E_{2}+\frac{\left(-z^{\prime} \operatorname{sen} \theta+\frac{r r^{\prime} \tau \cos \theta}{F}\right)}{\sqrt{1+\tau^{2} r^{2}}} E_{3} .
\end{aligned}
$$

Portanto, neste caso,

$$
\cos \vartheta=g_{k, \tau}\left(N, E_{1}\right)=\frac{r^{\prime}}{F \sqrt{1+\tau^{2} r^{2}}} .
$$

Também usaremos os campos de vetores $T$ e $J T$, descritos na Seção 2.2. Com este fim, passamos a descrevê-los usando a base $\left\{\Phi_{\theta}, \Phi_{s}\right\}$.

Como $T$ é a parte tangente de $E_{1}$, temos:

$$
\begin{aligned}
& g_{k, \tau}\left(T, \Phi_{\theta}\right)=g_{k, \tau}\left(E_{1}, \Phi_{\theta}\right)=-\frac{r^{2} \tau}{F}, \\
& g_{k, \tau}\left(T, \Phi_{s}\right)=g_{k, \tau}\left(E_{1}, \Phi_{s}\right)=z^{\prime}
\end{aligned}
$$

Escrevendo

$$
T=a \Phi_{\theta}+b \Phi_{s}
$$

obtemos o seguinte sistema

$$
\left\{\begin{array}{l}
g_{k, \tau}\left(T, \Phi_{\theta}\right)=a g_{k, \tau}\left(\Phi_{\theta}, \Phi_{\theta}\right)+b g_{k, \tau}\left(\Phi_{\theta}, \Phi_{s}\right), \\
g_{k, \tau}\left(T, \Phi_{s}\right)=a g_{k, \tau}\left(\Phi_{\theta}, \Phi_{s}\right)+b g_{k, \tau}\left(\Phi_{s}, \Phi_{s}\right),
\end{array}\right.
$$

cuja solução, juntamente com a condição (2.24), nos dá

$$
a=-\frac{r^{\prime 2} \tau}{F\left(1+r^{2} \tau^{2}\right)}, \quad b=\frac{z^{\prime}}{\left(1+r^{2} \tau^{2}\right)} .
$$

Usando (2.25), podemos também escrever $T$ com respeito à base ortonormal $\left\{E_{1}, E_{2}, E_{3}\right\}$ :

$$
\begin{aligned}
T= & \frac{\tau^{2} r^{2} r^{\prime 2}+F^{2} z^{\prime 2}}{F^{2}\left(1+r^{2} z^{2}\right)} E_{1}+\frac{r^{\prime}\left(\tau r r^{\prime} \operatorname{sen} \theta+F z^{\prime} \cos \theta\right)}{F^{2}\left(1+r^{2} \tau^{2}\right)} E_{2} \\
& +\frac{r^{\prime}\left(-\tau r r^{\prime} \cos \theta+F z^{\prime} \operatorname{sen} \theta\right)}{F^{2}\left(1+r^{2} \tau^{2}\right)} E_{3} .
\end{aligned}
$$


Quanto a $J T$, este pode ser obtido fazendo $N \wedge T$ e usando (2.24), o que resulta em

$$
J T=\frac{\tau r r^{\prime} \cos \theta-F z^{\prime} \operatorname{sen} \theta}{F \sqrt{1+\tau^{2} r^{2}}} E_{2}+\frac{\tau r r^{\prime} \operatorname{sen} \theta+F z^{\prime} \cos \theta}{F \sqrt{1+\tau^{2} r^{2}}} E_{3} .
$$

Com respeito à base $\left\{\Phi_{\theta}, \Phi_{s}\right\}$ temos

$$
J T=c \Phi_{\theta}+d \Phi_{s}
$$

onde $c$ e $d$ são obtidos a partir do sistema

$$
\left\{\begin{array}{l}
g_{k, \tau}\left(J T, \Phi_{\theta}\right)=c g_{k, \tau}\left(\Phi_{\theta}, \Phi_{\theta}\right)+d g_{k, \tau}\left(\Phi_{s}, \Phi_{\theta}\right), \\
g_{k, \tau}\left(J T, \Phi_{s}\right)=c g_{k, \tau}\left(\Phi_{\theta}, \Phi_{s}\right)+d g_{k, \tau}\left(\Phi_{s}, \Phi_{s}\right),
\end{array}\right.
$$

e possuem as expressões:

$$
c=\frac{F z^{\prime}}{r \sqrt{1+\tau^{2} r^{2}}}, \quad d=\frac{\tau r}{\sqrt{1+\tau^{2} r^{2}}}
$$

Nosso próximo objetivo é obter a função curvatura média da superfície $G_{X}$-invariante, o que é dado na proposição que segue.

Proposição 2.16. Seja $\mathcal{M}$ uma superfície $G_{X}$-invariante de um $B C V$-espaço $\mathcal{N}_{\kappa, \tau}$, com curva geradora $\gamma(s)=(r(s), z(s))$, parametrizada por comprimento de arco. Então a função curvatura média de $\mathcal{M}$ é dada por

$$
f=\left(\frac{1}{r}-\frac{\kappa}{4} r\right) \operatorname{sen} \sigma+\sigma^{\prime}
$$

sendo $\sigma$ o ângulo entre $\gamma^{\prime}$ e a direção $\frac{\partial}{\partial r}$ no espaço das órbitas.

Demonstração. Calcularemos a função curvatura média utilizando o Teorema de Redução 2.14 Ao longo de uma órbita principal $\xi$, temos:

$$
f=\kappa_{g}-D_{\mathbf{n}} \log \omega(\xi)
$$

onde $\kappa_{g}$ é a curvatura geodésica de $\gamma, D_{\mathbf{n}}$ é a derivação na direção normal e $\omega(\xi)$ é a função volume de $\xi$. 
Indicando por $\sigma$ o ângulo entre $\gamma^{\prime}$ e $\frac{\partial}{\partial r}$, a curvatura geodésica de $\gamma$ é dada por

$$
\begin{gathered}
k_{g}=\frac{1}{2 \sqrt{\widetilde{g}_{11} \widetilde{g}_{22}}}\left(\left(\widetilde{g}_{22}\right)_{r} z^{\prime}-\left(\widetilde{g}_{11}\right)_{z} r^{\prime}\right)+\sigma^{\prime} \\
=-\frac{\left(1+\frac{\kappa r^{2}}{4}\right) r \tau^{2} \operatorname{sen} \sigma}{1+r^{2} \tau^{2}}+\sigma^{\prime}
\end{gathered}
$$

Como $X$ é o gerador do grupo de isometrias que estamos considerando, temos

$$
\omega(\xi)=\sqrt{g_{\kappa, \tau}(X, X)}=\frac{r}{F} \sqrt{1+\tau^{2} r^{2}}
$$

Podemos obter o campo de vetores $\mathbf{n}$ normal a $\gamma$ ao projetarmos o campo de vetores $N$ sobre o espaço das órbitas. Resulta que

$$
\mathbf{n}=\left(-F \operatorname{sen} \sigma, \sqrt{1+\tau^{2} r^{2}} \cos \sigma\right)
$$

e, então,

$$
\begin{aligned}
D_{\mathbf{n}} \log \omega(\xi) & =\left.\frac{d}{d s} \log \omega(\xi+s \mathbf{n})\right|_{s=0} \\
& =\left.\frac{d}{d s} \log \left[\left(\frac{r-s(F \operatorname{sen} \sigma)}{1+\frac{\kappa}{4}(r-s F \operatorname{sen} \sigma)^{2}}\right) \sqrt{1+\tau^{2}(r-s F \operatorname{sen} \sigma)^{2}}\right]\right|_{s=0} \\
& =\frac{F\left(-4+r^{2}\left(\kappa-8 \tau^{2}\right)\right) \operatorname{sen} \sigma}{r\left(4+\kappa r^{2}\right)\left(1+r^{2} \tau^{2}\right)}
\end{aligned}
$$

Finalmente, substituindo as expressões (2.32) e (2.33) em (2.31), obtemos (2.30).

Observação 2.3. Posteriormente serão uteis as seguintes expressões

$$
\cos \sigma=\frac{\widetilde{g}\left(\gamma^{\prime}, \frac{\partial}{\partial r}\right)}{\sqrt{\widetilde{g}\left(\frac{\partial}{\partial r}, \frac{\partial}{\partial r}\right)}}=\frac{r^{\prime}}{F}, \quad \operatorname{sen} \sigma=\frac{z^{\prime}}{\sqrt{1+\tau^{2} r^{2}}}
$$

onde sen $\sigma$ foi obtido através da equação (2.24).

Neste ponto, de posse da expressão de $f$, a qual não depende de $\theta$, começamos a descrever as equações de biconservatividade. No que segue, usaremos a notação adotada na Seção 2.2 .

Proposição 2.17. Seja $\mathcal{M}$ uma superfície $G_{X}$-invariante de um $B C V$-espaço $\mathcal{N}_{\kappa, \tau}$, cuja função curvatura média seja $f$. Então, para que $\mathcal{M}$ seja biconservativa é necessário e suficiente que o 
seguinte sistema seja satisfeito:

$$
\left\{\begin{array}{l}
f^{\prime}\left[-\frac{2 b T(\cos \vartheta)}{\operatorname{sen}^{4} \vartheta}-\frac{2 d}{\operatorname{sen}^{2} \vartheta}\left(\frac{J T(\cos \vartheta)}{\operatorname{sen}^{2} \vartheta}+\tau\right)+\frac{b f}{\operatorname{sen}^{2} \vartheta}\right] \\
-2 f\left(4 \tau^{2}-\kappa\right) \cos \vartheta=0 \\
f^{\prime}\left[-2 b\left(\frac{J T(\cos \vartheta)}{\operatorname{sen}^{2} \vartheta}+\tau\right)+2 d \lambda+d f\right]=0
\end{array}\right.
$$

Demonstração. Para a demonstração, calculamos cada termo da equação (2.14). Temos

$$
\begin{aligned}
\operatorname{grad} f & =\frac{T(f)}{\|T\|_{g_{\kappa, \tau}}} \frac{T}{\|T\|_{g_{\kappa, \tau}}}+\frac{J T(f)}{\|J T\|_{g_{\kappa, \tau}}} \frac{J T}{\|J T\|_{g_{\kappa, \tau}}} \\
& =\frac{b \Phi_{s}(f) T+d \Phi_{s}(f) J T}{\|J T\|_{g_{\kappa, \tau}}^{2}}
\end{aligned}
$$

ou seja, indicando $\Phi_{s}(f)=f^{\prime}$, já que $f$ depende apenas de $s$, temos

$$
\operatorname{grad} f=\frac{f^{\prime}(b T+d J T)}{\|J T\|_{g_{\kappa, \tau}}^{2}} .
$$

Além disso, usando (2.10) vemos que

$$
\begin{aligned}
A(\operatorname{grad} f) & =\frac{f^{\prime}(b A(T)+d A(J T))}{\|J T\|_{g_{\kappa, \tau}}^{2}} \\
& =\frac{b f^{\prime}}{\operatorname{sen}^{2} \vartheta}\left(-\frac{T(\cos \vartheta)}{\operatorname{sen}^{2} \vartheta} T-\left(\frac{J T(\cos \vartheta)}{\operatorname{sen}^{2} \vartheta}+\tau\right) J T\right) \\
& +\frac{d f^{\prime}}{\operatorname{sen}^{2} \vartheta}\left(-\left(\frac{J T(\cos \vartheta)}{\operatorname{sen}^{2} \vartheta}+\tau\right) T+\lambda J T\right) .
\end{aligned}
$$

Portanto, levando em conta (2.16), obtemos as condições do sistema (2.35).

Observação 2.4. Para manipular as expressões do sistema (2.35), serão úteis as expressões

$$
\begin{aligned}
T(\cos \vartheta) & =a \Phi_{\theta}(\cos \vartheta)+b \Phi_{s}(\cos \vartheta) \\
& =a \Phi_{\theta}\left(\frac{r^{\prime}}{F \sqrt{1+\tau^{2} r^{2}}}\right)+b \Phi_{s}\left(\frac{r^{\prime}}{F \sqrt{1+\tau^{2} r^{2}}}\right) \\
& =b\left(\frac{r^{\prime}}{F \sqrt{1+\tau^{2} r^{2}}}\right)^{\prime}
\end{aligned}
$$


$e$

$$
\begin{aligned}
J T(\cos \vartheta) & =c \Phi_{\theta}(\cos \vartheta)+d \Phi_{s}(\cos \vartheta) \\
& =c \Phi_{\theta}\left(\frac{r^{\prime}}{F \sqrt{1+\tau^{2} r^{2}}}\right)+d \Phi_{s}\left(\frac{r^{\prime}}{F \sqrt{1+\tau^{2} r^{2}}}\right) \\
& =d\left(\frac{r^{\prime}}{F \sqrt{1+\tau^{2} r^{2}}}\right)^{\prime} .
\end{aligned}
$$

Como estamos interessados em superfícies que não sejam de curvatura média constante, pois estas já foram tratadas na Seção 2.4 a segunda condição de (2.35) dá

$$
-2 b\left(\frac{J T(\cos \vartheta)}{\operatorname{sen}^{2} \vartheta}+\tau\right)+2 d \lambda+d f=0
$$

que, por cálculos diretos, se torna

$$
\frac{\tau\left(3 f r \sqrt{1+\tau^{2} r^{2}}-2 z^{\prime}\right)}{1+\tau^{2} r^{2}}=0 .
$$

Logo, temos duas possibilidades:

1. $\tau \neq 0$, que nos dá

$$
f=\frac{2 z^{\prime}}{3 r \sqrt{1+\tau^{2} r^{2}}}
$$

2. $\tau=0$.

\subsubsection{O caso $\tau \neq 0$}

O estudo deste caso fica sintetizado pelo teorema seguinte.

Teorema 2.18. Seja $\mathcal{M}$ uma superfície $G_{X}$-invariante biconservativa de um $B C V$-espaço $\mathcal{N}_{\kappa, \tau}$, que não seja um espaço forma e com $\tau \neq 0$. Então $\mathcal{M}$ será biconservativa se, e somente se, for um cilindro de Hopf sobre uma curva de curvatura geodésica constante.

Demonstração. Começamos usando (2.34) para reescrever (2.37) na forma

$$
f=\frac{2 \operatorname{sen} \sigma}{3 r}
$$


Observe que sen $\sigma \neq 0$, já que $f$ é não nula. Dessa forma, comparando (2.38) com (2.30) obtemos

$$
\frac{\sigma^{\prime}}{\operatorname{sen} \sigma}=\left(\frac{-1}{3 r}+\frac{\kappa r}{4}\right)
$$

Logo, multiplicando ambos os lados por

$$
\cos \sigma=\frac{4 r^{\prime}}{4+\kappa r^{2}}
$$

obtemos

$$
\sigma^{\prime} \cot \sigma=\frac{-4+3 \kappa r^{2}}{3 r\left(4+\kappa r^{2}\right)} r^{\prime}
$$

que, integrando, nos dá

$$
\log (\operatorname{sen} \sigma)=\frac{1}{3}\left(-\log (r)+2 \log \left(4+\kappa r^{2}\right)\right)+C_{0}
$$

onde $C_{0}$ é uma constante de integração. Consequentemente temos

$$
\operatorname{sen} \sigma=C_{1} \frac{\left(4+\kappa r^{2}\right)^{2 / 3}}{r^{1 / 3}}
$$

sendo $C_{1}$ uma constante positiva.

De (2.39) e também, usando

$$
\cos \sigma=\frac{4 r^{\prime}}{4+k r^{2}}
$$

obtemos

$$
r^{\prime}=\frac{\left(4+k r^{2}\right) \sqrt{r^{2 / 3}-C_{1}^{2}\left(4+k r^{2}\right)^{4 / 3}}}{4 r^{1 / 3}} .
$$

Agora vamos considerar a primeira equação de (2.35). Devido a (2.27) e a (2.28) podemos reescrevê-la na forma

$$
f^{\prime}\left(b f-\frac{2\left(b^{2}+d^{2}\right)}{\operatorname{sen}^{2} \sigma}(\cos \vartheta)^{\prime}-2 d \tau\right)-2 f\left(4 \tau^{2}-\kappa\right) \cos \vartheta \operatorname{sen}^{2} \vartheta=0 .
$$

Em seguida, usando (2.29), 2.38), o fato de que

$$
b=\frac{z^{\prime}}{\sqrt{1+\tau^{2} r^{2}}}=\frac{\operatorname{sen} \sigma}{\sqrt{1+\tau^{2} r^{2}}}
$$


e observando que $b^{2}+d^{2}=\operatorname{sen}^{2} \vartheta$, obtemos

$$
\left(\frac{2 \operatorname{sen} \sigma}{3 r}\right)^{\prime}\left(\frac{2 \operatorname{sen}^{2} \sigma-6 r^{2} \tau^{2}}{3 r \sqrt{1+\tau^{2} r^{2}}}-2(\cos \vartheta)^{\prime}\right)-\frac{4 \operatorname{sen} \sigma}{3 r}\left(4 \tau^{2}-\kappa\right) \cos \vartheta \operatorname{sen}^{2} \vartheta=0
$$

Como consequência de (2.39) e (2.40), segue que

$$
\begin{aligned}
\cos \vartheta & =\frac{\sqrt{r^{2 / 3}-C_{1}^{2}\left(4+k r^{2}\right)^{4 / 3}}}{r^{1 / 3} \sqrt{1+\tau^{2} r^{2}}} ; \\
(\cos \vartheta)^{\prime} & =\frac{\left(4+k r^{2}\right)\left(-3 \tau^{2} r^{8 / 3}+C_{1}^{2}\left(4+k r^{2}\right)^{1 / 3}\left(4+r^{2}\left(-3 \kappa+16 \tau^{2}\right)\right)\right)}{12 r^{5 / 3}\left(1+\tau^{2} r^{2}\right)^{3 / 2}} ; \\
\operatorname{sen}^{2} \vartheta & =1-\frac{r^{2 / 3}-C_{1}^{2}\left(4+k r^{2}\right)^{4 / 3}}{r^{2 / 3}\left(1+\tau^{2} r^{2}\right)} ; \\
\left(\frac{2 \operatorname{sen} \sigma}{3 r}\right)^{\prime} & =-\frac{8 C_{1}\left(4+k r^{2}\right)^{2 / 3} \sqrt{r^{2 / 3}-C_{1}^{2}\left(4+k r^{2}\right)^{4 / 3}}}{9 r^{8 / 3}} .
\end{aligned}
$$

Finalmente, substituindo estas expressões em (2.41) temos

$$
\frac{8 C_{1}\left(\kappa-4 \tau^{2}\right)\left(4+k r^{2}\right)^{2 / 3}\left(\tau^{2} r^{8 / 3}+C_{1}^{2}\left(4+k r^{2}\right)^{4 / 3}\right) \cos \sigma}{9 r^{2}\left(1+\tau^{2} r^{2}\right)^{3 / 2}}=0 .
$$

Sendo os demais termos da multiplicação no numerador não nulos, concluímos que $\cos \sigma=0$. Logo $r$ é constante e, devido a (2.26), temos $\cos \vartheta=0$. O resultado segue então do Teorema 2.13 ,

\subsubsection{O caso $\tau=0$}

Neste caso temos

$$
a=0, \quad b=z^{\prime}, \quad c=\frac{F z^{\prime}}{r}, \quad d=0,
$$

de forma que as expressões de $T$ e $J T$ ficam

$$
T=z^{\prime} \Phi_{s}, \quad J T=\frac{F z^{\prime}}{r} \Phi_{\theta} .
$$

Além disso, a condição de que $\gamma$ seja parametrizada pelo comprimento de arco, dada em (2.24) se torna

$$
\frac{r^{\prime 2}}{F^{2}}+z^{\prime 2}=1
$$


logo, de (2.26) segue que

$$
\cos \vartheta=\frac{r^{\prime}}{F} \quad \text { e } \quad \operatorname{sen} \vartheta=z^{\prime}
$$

Já (2.34) nos dá

$$
\cos \sigma=\frac{r^{\prime}}{F} \quad \text { e } \quad \operatorname{sen} \sigma=z^{\prime}
$$

donde concluímos que $\sigma=\vartheta$. As superfícies para este caso ficam, então, caracterizadas pelo seguinte teorema.

Teorema 2.19. Seja $\mathcal{M}$ uma superfície $G_{X}$-invariante de um $B C V$-espaço que possua $\tau=0$, cuja função curvatura média é dada por $f$. Então $\mathcal{M}$ será biconservativa se, e somente se, satisfizer o sistema

$$
\left\{\begin{array}{l}
f=\left(\frac{1}{r}-\frac{\kappa}{4} r\right) \operatorname{sen} \vartheta+\vartheta^{\prime}, \\
f^{\prime}\left(2 \vartheta^{\prime}+f\right)+2 \kappa f \cos \vartheta \operatorname{sen} \vartheta=0 .
\end{array}\right.
$$

Demonstração. Como a condição (2.36) é automaticamente satisfeita para este caso, temos como única restrição a primeira das equações de biconservatividade dada em (2.35), que pode ser reescrita usando (2.42) e (2.43). Desta forma, considerando a expressão de $f$ dada em (2.30) obtemos o sistema (2.45).

Chamamos a atenção para o fato de que é possível obter uma EDO sobre a função $r$, cujas soluções podem ser utilizadas para determinar $z$, de forma que possamos obter explicitamente a parametrização da curva geradora $\gamma$. Para isto, escrevemos

$$
z^{\prime}=\sin \vartheta=\sqrt{1-\cos ^{2} \vartheta}=\sqrt{1-\frac{16 r^{\prime 2}}{\left(4+\kappa r^{2}\right)^{2}}}
$$

em seguida usando

$$
\vartheta^{\prime}=-\frac{(\cos \vartheta)^{\prime}}{\sin \vartheta}
$$


na primeira equação de (2.45), podemos obter a seguinte expressão para $f$ como função apenas de $r$ e suas derivadas:

$$
f=\frac{r\left(k r\left(-k r^{2}\left(k r^{2}+4\right)+48\left(r^{\prime}\right)^{2}+16\right)-16\left(k r^{2}+4\right) r^{\prime \prime}\right)-64\left(\left(r^{\prime}\right)^{2}-1\right)}{4 r\left(k r^{2}+4\right) \sqrt{\left(k r^{2}+4\right)^{2}-16\left(r^{\prime}\right)^{2}}} .
$$

Finalmente podemos obter a EDO que determina $r$ substituindo $f, \cos \vartheta \sin \vartheta$ e $\vartheta^{\prime}$ na segunda equação de (2.45). A equação resultante é

$$
\begin{aligned}
& -32 \kappa r^{2} r^{\prime}\left(\kappa^{2} r^{4}+8 \kappa r^{2}-16 r^{2}+16\right)^{2} \\
& \left(r\left(\kappa r\left(\kappa^{2} r^{4}+4 \kappa r^{2}-48 r^{2}-16\right)+16\left(\kappa r^{2}+4\right) r^{\prime \prime}\right)+64\left(\left(r^{\prime}\right)^{2}-1\right)\right)+ \\
& \left(\kappa^{3} r^{6}+4 \kappa^{2} r^{4}+48 \kappa r^{3} r^{\prime \prime}-16 r^{2}\left(7 \kappa\left(r^{\prime}\right)^{2}+\kappa\right)+192 r r^{\prime \prime}+64\left(\left(r^{\prime}\right)^{2}-1\right)\right) \\
& {\left[-256\left(3 \kappa^{2} r^{4}-24 \kappa r^{2}-16\right)\left(r^{\prime}\right)^{5}+32\left(\kappa r^{2}+4\right)\left(r^{\prime}\right)^{3}\right.} \\
& \left(3 \kappa^{3} r^{6}-4 \kappa^{2} r^{4}+24 \kappa r^{3} r^{\prime \prime}-80 \kappa r^{2}-32 r r^{\prime \prime}-64\right)+ \\
& \left(\kappa r^{2}+4\right)^{2} r^{\prime}\left(\kappa^{4} r^{8}+16 \kappa^{3} r^{6}-112 \kappa^{2} r^{5} r^{\prime \prime}+96 \kappa^{2} r^{4}-384 \kappa r^{3} r^{\prime \prime}\right. \\
& \left.+256 r^{2}\left(\kappa+\left(r^{\prime \prime}\right)^{2}\right)+256 r r^{\prime \prime}+256\right)+ \\
& \left.16 r^{2} r^{(3)}\left(\kappa r^{2}+4\right)^{4}-256 r^{2} r^{(3)}\left(\kappa r^{2}+4\right)^{2} r^{\prime 2}\right]=0 .
\end{aligned}
$$

O caso $\tau=\kappa=0$

Um caso importante a ser analisado é o caso $\tau=\kappa=0$, em que o BCV-espaço é o espaço Euclidiano tridimensional. Neste caso, a segunda equação do sistema (2.45) nos dá que $f$ é constante, ou

$$
2 \vartheta^{\prime}+f=0
$$

Além disso, substituindo $\kappa=0$ na primeira equação de (2.45), obtemos

$$
f=\frac{1}{r} \operatorname{sen} \vartheta+\vartheta^{\prime}
$$


Combinando essas duas equações e usando (2.44), que implica que $\vartheta=\arcsin z^{\prime}$, obtemos

$$
\frac{z^{\prime}}{r}+3 \frac{z^{\prime \prime}}{\sqrt{1-z^{\prime 2}}}=0
$$

Em seguida, usando a condição (2.24) conseguimos

$$
3 r^{\prime \prime} r=1-r^{2}
$$

Para integrarmos esta equação, fazemos a substituição $y=r^{\prime 2}$, donde

$$
\frac{y^{\prime}}{1-y}=\frac{2}{3} \frac{r^{\prime}}{r}
$$

e, portanto,

$$
\frac{1}{1-y}=r^{(2 / 3)}
$$

Finalmente, substituindo $y=r^{\prime 2}$ resulta

$$
\frac{r^{\prime}}{\sqrt{1-r^{(3 / 2)} c}}=1
$$

onde $c$ é uma constante de integração.

Observação 2.5. A equação (2.47) também pode ser obtida substituindo $\kappa=0$ em (2.46), o que nos dá:

$$
\begin{aligned}
& \left(3 r r^{\prime \prime}+\left(r^{\prime}\right)^{2}-1\right)\left[r^{\prime}\left(r^{2}\left(r^{\prime \prime}\right)^{2}+\left(\left(r^{\prime}\right)^{2}-1\right)^{2}-r\left(\left(r^{\prime}\right)^{2}-1\right) r^{\prime \prime}\right)\right. \\
& \left.-r^{2} r^{(3)}\left(\left(r^{\prime}\right)^{2}-1\right)\right]=0
\end{aligned}
$$

$e$, então, observamos que o termo entre colchetes é exatamente o numerador de $f^{\prime}$ para $\kappa=0$. Logo, se f não for constante, obtemos (2.47).

Observação 2.6. $O$ caso $\tau=\kappa=0$ foi estudado em [50], onde os autores consideram a função $J$ dada por

$$
J=r^{(1 / 3)} \operatorname{sen} \vartheta
$$

e demonstram que esta função é contante ao longo das curvas soluções de (2.47). Em seguida, usam este fato para mostrar que a curva geradora da superfície em questão é do tipo catenária. 


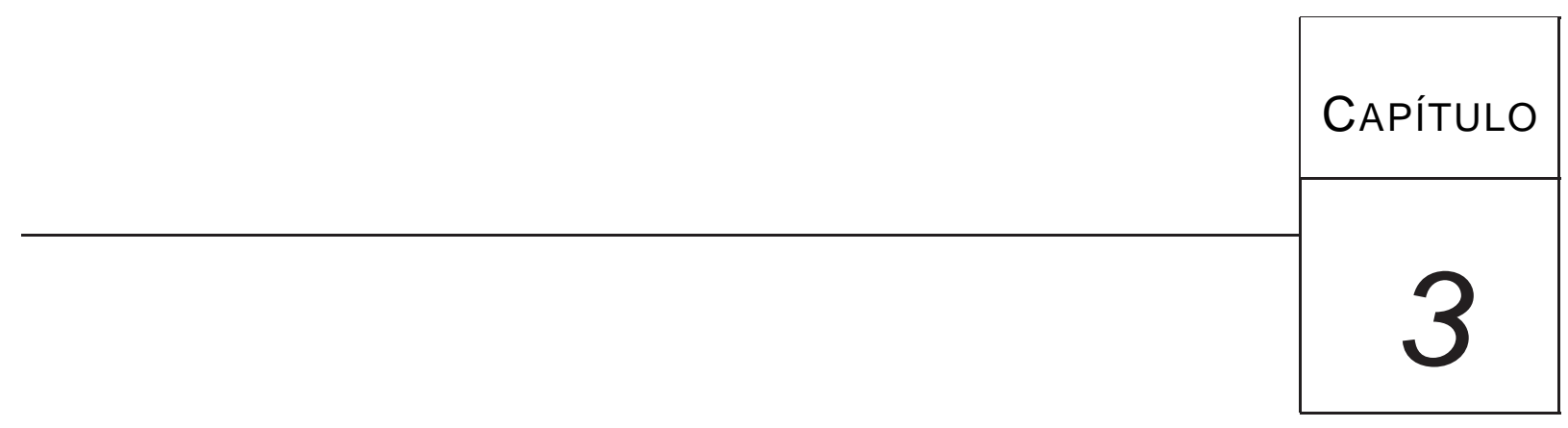

\section{Superfícies com aplicação de Gauss}

bi-harmônicas

\subsection{Introdução}

Uma das ferramentas mais úteis no estudo de subvariedades orientadas do espaço Euclidiano é a aplicação de Gauss associada a esta. Através da aplicação de Gauss diversas propriedades e características extrínsecas da subvariedade podem ser determinadas. Um exemplo de aplicação desta poderosa ferramenta é o Teorema de Ruh-Vilms (ver [64]), que diz que a aplicação de Gauss

$$
\gamma: \mathcal{M}^{m} \subset \mathbb{R}^{m+n} \rightarrow G(m, n)
$$


que a cada ponto $p$ de $\mathcal{M}$ associa o espaço tangente $T_{p} \mathcal{M}$, visto como um ponto da variedade Grassmanniana dos subespaços $m$-dimensionais orientados de $\mathbb{R}^{m+n}$, é harmônica se, e somente se, o campo curvatura média de $\mathcal{M}$ é paralelo.

Uma generalização natural para o Teorema de Ruh-Vilms é o estudo das subvariedades do $\mathbb{R}^{m+n}$ que possuem aplicação de Gauss bi-harmônica. Este estudo foi iniciado por A. Balmuş, S. Montaldo e C. Oniciuc em [6], onde é dada a caracterização destas subvariedades através do seguinte teorema:

Teorema 3.1 ( [6]]). A aplicação de Gauss associada a uma subvariedade m-dimensional $\mathcal{M}$ de $\mathbb{R}^{m+n}$ é bi-harmônica própria se, e somente se, $\nabla^{\perp} H \neq 0$ e

$$
\begin{aligned}
& \nabla_{X}^{\perp} \Delta^{\perp} H-m \nabla_{A_{H}(X)}^{\perp}+\operatorname{tr} B\left(2 A_{\nabla_{(\cdot)}^{\perp} H}(X)-A_{\nabla_{(X)}^{\perp} H}(\cdot), \cdot\right) \\
& +2 \operatorname{tr} \mathrm{R}^{\perp}(\cdot, X) \nabla_{(\cdot)}^{\perp} H+\operatorname{tr}\left(\nabla_{(\cdot)}^{\perp} \mathrm{R}^{\perp}\right)(\cdot, X) H=0,
\end{aligned}
$$

para todo campo de vetores diferenciável $X \in T \mathcal{M}$. Aqui A denota o operador de Weingarten, $H$ o campo curvatura média de $\mathcal{M}$ em $\mathbb{R}^{m+n}, B$ a segunda fundamental $e \nabla^{\perp}, \Delta^{\perp} e \mathrm{R}^{\perp}$ denotam, respectivamente, a conexão, o Laplaciano generalizado e o tensor de curvatura agindo no fibrado normal de $\mathcal{M}$.

Para o caso de hipersuperfícies a equação (3.1) se simplifica, ficando na forma dada no teorema que segue.

Teorema 3.2 ( [6]). A aplicação de Gauss para uma hipersuperfície não mínima $\mathcal{M}^{m} \subset \mathbb{R}^{m+1} e ́$ bi-harmônica própria se, e somente se, $f \neq 0$ e

$$
\Delta \operatorname{grad} f+A^{2}(\operatorname{grad} f)-\|A\|^{2} \operatorname{grad} f=0,
$$

sendo $\Delta$ o Laplaciano generalizado em TM, enquanto $f$ e A são a função curvatura média e o operador de forma, respectivamente.

Ainda em [6], os autores consideram hipercones no espaço Euclidiano, obtendo exemplos de superfícies com aplicação de Gauss bi-harmônica própria. São estudados os hipercones gerados 
por hipersuperfícies de curvatura média constante em esferas e, em particular, por hiperesferas. Além disso, é demonstrada a não existência de cones em $\mathbb{R}^{3}$ com aplicação de Gauss bi-harmônica própria.

Neste capítulo estudaremos a equação (3.2) no caso especial em que a superfície faz um ângulo constante com uma direção pré-fixada, a fim de obtermos exemplos de superfícies com aplicação de Gauss bi-harmônica, com o intuito de compreender melhor tal condição.

\subsection{Superfícies de ângulo constante com aplicação de Gauss bi-harmônica}

Considere uma superfície $\mathcal{M}$ de $\mathbb{R}^{3}$, orientada e cujo campo de vetores normais faz um ângulo constante com uma direção prefixada. Sem perda de generalidade, podemos tomar a direção fixa como sendo o campo $\frac{\partial}{\partial z}$. Como salientado na Observação 2.1, embora as técnicas descritas na Seção 2.2 tenham sido desenvolvidas e aplicadas para os BCV-espaços com grupo de isometria de dimensão 4, podemos proceder de forma análoga para as superfícies isometricamente imersas no $\mathbb{R}^{3}$. Desta forma, se $N$ é um campo de vetores unitário e normal à superfície $\mathcal{M}$, consideramos que ele faz ângulo constante $\vartheta \operatorname{com} \frac{\partial}{\partial z}$, o que nos dá

$$
\left|\left\langle\frac{\partial}{\partial z}, N\right\rangle\right|=\cos \vartheta, \quad \vartheta \in[0, \pi / 2]
$$

Então, projetando $\frac{\partial}{\partial z}$ no plano tangente e $\mathcal{M}$ temos

$$
\frac{\partial}{\partial z}=T+\cos \vartheta N
$$

onde $T$ é tangente à superfície e satisfaz $\langle T, T\rangle=\operatorname{sen}^{2} \vartheta$.

Usaremos $\nabla$ para denotar a conexão de Levi-Civita em $\mathcal{M}$.

Proposição 3.3. Para um campo $X$ tangente a $\mathcal{M} \subset \mathbb{R}^{3}$ qualquer, vale

$$
\nabla_{X} T=\cos \vartheta A X, \quad\langle A X, T\rangle=0 .
$$


Demonstração. A demonstração é feita de forma inteiramente análoga à da Proposição 2.5, com as devidas considerações (para $\mathbb{R}^{3}$ as constantes $\tau$ e $\kappa$ são nulas).

Agora, considere a base $\{T, J T\}$, com $J T:=N \wedge T$. Tomando

$$
e_{1}=\frac{T}{\operatorname{sen} \vartheta} \quad \text { e } \quad e_{2}=\frac{J T}{\operatorname{sen} \vartheta}
$$

obtemos uma base ortonormal para o fibrado tangente $T \mathcal{M}$. Então, definindo

$$
\lambda:=\left\langle A e_{2}, e_{2}\right\rangle
$$

e usando (3.4), obtemos a matriz associada a $A$ com respeito à base $\left\{e_{1}, e_{2}\right\}$,

$$
A=\left(\begin{array}{ll}
0 & 0 \\
0 & \lambda
\end{array}\right) \text {. }
$$

Dessa forma a função curvatura média é dada por

$$
f=\operatorname{tr} A=\lambda \text {. }
$$

Com o uso das fórmulas (3.4), obtemos as componentes da conexão:

$$
\begin{array}{ll}
\nabla_{e_{1}} e_{1}=0, & \nabla_{e_{2}} e_{1}=f \cot \vartheta e_{2}, \\
\nabla_{e_{1}} e_{2}=0, & \nabla_{e_{2}} e_{2}=-f \cot \vartheta e_{1} .
\end{array}
$$

Nos servirão, também, as equações de Gauss e Codazzi que para este caso assumem a forma:

$$
\begin{gathered}
K=\operatorname{det} A \\
\nabla_{X} A(Y)-\nabla_{Y} A(X)=A[X, Y]
\end{gathered}
$$

para quaisquer $X, Y \in T \mathcal{M}$.

Com as notações estabelecidas acima, vale a proposição que segue.

Proposição 3.4. Seja $\mathcal{M}$ uma superfície orientada em $\mathbb{R}^{3}$ com ângulo constante $\vartheta$. Então, sua função curvatura média $f$ satisfaz.

$$
e_{1}(f)=-f^{2} \cot \vartheta
$$


Demonstração. Para a demonstração utilizamos a equação de Codazzi fazendo $X=e_{1}$ e $Y=e_{2}$ obtemos:

$$
\begin{aligned}
\nabla_{e_{1}} A\left(e_{2}\right)-\nabla_{e_{2}} A\left(e_{1}\right) & =A\left[e_{1}, e_{2}\right] \\
\nabla_{e_{1}}\left(f e_{2}\right) & =A\left(-f \cot \vartheta e_{2}\right) \\
e_{1}(f) e_{2} & =-f^{2} \cot \vartheta e_{2},
\end{aligned}
$$

de onde segue a tese da proposição.

Observação 3.1. A equação de Gauss nos dá a condição $e_{1}(f) \cot \vartheta=-f^{2} \cot ^{2} \vartheta$, que também pode ser obtida a partir da expressão dada na proposição acima.

Em seguida, consideraremos o caso em que a superfície $\mathcal{M}$ possui aplicação de Gauss biharmônica.

Proposição 3.5. Seja $\mathcal{M} \subset \mathbb{R}^{3}$ uma superfície com ângulo constante $\vartheta$. Para que a aplicação de Gauss associada a M seja bi-harmônica é necessário e suficiente que seja satisfeito o sistema

$$
\left\{\begin{array}{l}
4 \cot \vartheta f e_{2} e_{2}(f)+3 \cot \vartheta e_{2}(f)^{2}+3 \cot ^{3} \vartheta f^{4}+\cot \vartheta f^{4}=0 \\
e_{2} e_{2} e_{2}(f)+3 \cot ^{2} \vartheta f^{2} e_{2}(f)=0
\end{array}\right.
$$

Demonstração. Para a prova calcularemos cada termo da equação (3.2). Temos:

$$
\begin{aligned}
\operatorname{grad} f & =e_{1}(f) e_{1}+e_{2}(f) e_{2} \\
& =-f^{2} \cot \vartheta e_{1}+e_{2}(f) e_{2} .
\end{aligned}
$$

Consequentemente, usando (3.5) obtemos

$$
\begin{aligned}
\|A\|^{2} \operatorname{grad} f & =\left(\sum_{i, j=1}^{2}\left\langle A\left(e_{i}\right), e_{j}\right\rangle^{2}\right)\left(-f^{2} \cot \vartheta e_{1}+e_{2}(f) e_{2}\right) \\
& =-f^{4} \cot \vartheta e_{1}+f^{2} e_{2}(f) e_{2}
\end{aligned}
$$

e, também,

$$
A^{2}(\operatorname{grad} f)=f^{2} e_{2}(f) e_{2} .
$$


Para o termo $\Delta(\operatorname{grad} f)$ nos será útil a expressão

$$
e_{1} e_{2}(f)=\left[e_{1}, e_{2}\right](f)+e_{2} e_{1}(f)=\left(\nabla_{e_{1}} e_{2}-\nabla_{e_{2}} e_{1}\right)(f)+e_{2}\left(-f^{2} \cot \vartheta\right)=-3 \cot \vartheta f e_{2}(f) .
$$

Calculamos

$$
\Delta(\operatorname{grad} f)=-\left\{\nabla_{e_{1}} \nabla_{e_{1}}-\nabla_{\nabla_{e_{1}} e_{1}}+\nabla_{e_{2}} \nabla_{e_{2}}-\nabla_{\nabla_{e_{2}} e_{2}}\right\}(\operatorname{grad} f),
$$

fazendo de forma individual as derivações covariantes e em seguida efetuando a soma. Sendo assim temos

$$
\begin{aligned}
\nabla_{e_{1}} \nabla_{e_{1}}(\operatorname{grad} f) & =\nabla_{e_{1}}\left(-2 \cot \vartheta f e_{1}(f) e_{1}+e_{1} e_{2}(f) e_{2}\right) \\
& =\nabla_{e_{1}}\left(-2 \cot ^{2} \vartheta f^{3} e_{1}-3 \cot \vartheta f e_{2}(f) e_{2}\right) \\
& =6 \cot ^{2} \vartheta f^{2} e_{1}(f) e_{1}-3 \cot \vartheta\left(e_{1}(f) e_{2}(f)+f e_{1} e_{2}(f)\right) e_{2} \\
& =-6 \cot ^{3} \vartheta f^{4} e_{1}+12 \cot ^{2} \vartheta f^{2} e_{2}(f) e_{2},
\end{aligned}
$$

enquanto que $\nabla_{e_{1}} e_{1}=0$ nos fornece

$$
\nabla_{\nabla_{e_{1}} e_{1}}(\operatorname{grad} f)=0
$$

Além disso,

$$
\begin{aligned}
\nabla_{e_{2}} \nabla_{e_{2}}(\operatorname{grad} f)= & \nabla_{e_{2}}\left(-2 \cot \vartheta f e_{2}(f) e_{1}-\cot \vartheta f^{2}\left(\cot \vartheta f e_{2}\right)+e_{2} e_{2}(f) e_{2}\right) \\
= & \left(-4 \cot \vartheta f e_{2} e_{2}(f)-3 \cot \vartheta e_{2}(f)^{2}+f^{4} \cot ^{3} \vartheta\right) e_{1} \\
& +\left(-6 \cot ^{2} \vartheta f^{2} e_{2}(f)+e_{2} e_{2} e_{2}(f)\right) e_{2},
\end{aligned}
$$

e, por fim,

$$
\begin{aligned}
\nabla_{\nabla_{e_{2}} e_{2}}(\operatorname{grad} f) & =-\cot \vartheta f\left(2 \cot ^{2} \vartheta f^{3} e_{1}-3 \cot \vartheta f e_{2}(f) e_{2}\right) \\
& =-2 \cot ^{3} \vartheta f^{4} e_{1}+3 \cot ^{2} \vartheta f^{2} e_{2}(f) e_{2} .
\end{aligned}
$$

Portanto

$$
\begin{aligned}
\Delta(\operatorname{grad} f) & =\left(4 \cot \vartheta f e_{2} e_{2}(f)+3 \cot \vartheta e_{2}(f)^{2}+3 \cot ^{3} \vartheta f^{4}\right) e_{1} \\
& -\left(e_{2} e_{2} e_{2}(f)+3 \cot ^{2} \vartheta f^{2} e_{2}(f)\right) e_{2} .
\end{aligned}
$$

O resultado segue substituindo as expressões obtidas acima na equação (3.2) considerando as componentes na direção de $e_{1}$ e de $e_{2}$. 
A partir da Proposição 3.5 conseguimos classificar as superfícies de $\mathbb{R}^{3}$ com ângulo constante que possuem aplicação de Gauss bi-harmônica.

Teorema 3.6. Seja $\mathcal{M} \subset \mathbb{R}^{3}$ uma superfície com ângulo constante $\vartheta$. Se $\mathcal{M}$ possuir aplicação de Gauss bi-harmônica, então $\mathcal{M}$ será uma parte de um cilindro sobre uma clotóide.

Demonstração. Para a prova deste teorema usamos o sistema (3.7), dividindo o estudo dele em dois casos: o caso em que $\cot \vartheta=0$, e o caso em quem $\cot \vartheta \neq 0$.

1) Se $\cot \vartheta=0$, então $N$ é normal ao campo $\frac{\partial}{\partial z}$, de forma que (3.3) implica em

$$
e_{1}=\frac{\partial}{\partial z}
$$

Concluímos que a superfície $\mathcal{M}$ é um cilindro ortogonal ao plano-xy, sobre a curva integral do campo de vetores $e_{2}$, que denotaremos por $\gamma(s)$.

Do sistema (3.7), resulta que

$$
e_{2} e_{2} e_{2}(f)=0
$$

Já a condição (3.6) se torna

$$
e_{1}(f)=0
$$

donde podemos concluir que

$$
f=C_{0}+C_{1} s+C_{2} s^{2} .
$$

Além disso, as conexões do espaço ambiente $\mathbb{R}^{3}$ se tornam

$$
\begin{array}{ll}
\bar{\nabla}_{e_{1}} e_{1}=0, & \bar{\nabla}_{e_{2}} e_{1}=0, \\
\bar{\nabla}_{e_{1}} e_{2}=0, & \bar{\nabla}_{e_{2}} e_{2}=f N,
\end{array}
$$

de forma que a curva $\gamma$ possui curvatura geodésica igual a $f$. Uma curva do espaço Euclidiano com tal curvatura é conhecida como clotóide ou espiral de Cornu. Destacamos que a superfície do cilindro assim obtida é a mesma descrita em [6]. 
2) No caso em que $\cot \vartheta \neq 0$, as equações (3.7) dão

$$
\left\{\begin{array}{l}
4 f e_{2} e_{2}(f)+3 e_{2}(f)^{2}+\left(3 \cot ^{2} \vartheta+1\right) f^{4}=0 ; \\
e_{2} e_{2} e_{2}(f)=-3 \cot ^{2} \vartheta f^{2} e_{2}(f) .
\end{array}\right.
$$

Usando a segunda destas, obtemos

$$
e_{2} e_{2}(f)=-\cot ^{2} \vartheta f^{3}+C_{0}
$$

sendo $C_{0}$ uma função definida sobre $\mathcal{M}$ tal que $e_{2}\left(C_{0}\right)=0$. Combinando a primeira equação de (3.8) com (3.9) resulta

$$
3 e_{2}(f)^{2}+\left(-\cot ^{2} \vartheta+1\right) f^{4}+4 C_{0} f=0,
$$

que, ao ser derivada com respeito a $e_{2}$, fornece

$$
6 e_{2}(f) e_{2} e_{2}(f)+4\left(-\cot ^{2} \vartheta+1\right) f^{3} e_{2}(f)+4 C_{0} e_{2}(f)=0 .
$$

Supondo $e_{2}(f)=0$, da primeira equação do sistema (3.8) obtemos

$$
\left(3 \cot ^{2} \vartheta+1\right) f^{4}=0
$$

donde $f=0$, o que nos leva à uma contradição. Portanto, como $e_{2}(f) \neq 0$, substituindo a expressão de $e_{2} e_{2}(f)$ dada em (3.9) na equação (3.11) obtemos

$$
\left(-10 \cot ^{2} \vartheta+4\right) f^{3}=-10 C_{0}
$$

Derivando esta última equação com respeito a $e_{2}$, resulta que $\cot ^{2} \vartheta=2 / 5$, e logo $C_{0}=0$. Substituindo esses valores em (3.10) obtemos

$$
3 e_{2}(f)^{2}+\frac{3}{5} f^{4}=0
$$

o que é um absurdo, já que cada termo do lado esquerdo da equação é estritamente positivo. Concluímos que $\cot \vartheta \neq 0$ não pode ocorrer. 


\begin{tabular}{|l|l|}
\hline CAPÍtulo \\
\hline 1 \\
\hline 1
\end{tabular}

\section{Superfícies de ângulo constante no} grupo especial linear

Nos últimos anos muito avanço tem sido feito para entender a geometria de superfícies cujo campo de vetores normal unitário faz um ângulo constante com uma direção fixa do espaço ambiente. Essas superfícies são chamadas de superfícies do tipo hélice ou superfícies de ângulo constante e vêm sendo estudadas em grande parte das variedades Riemannianas tridimensionais. Em [16] Cermelli e Di Scala analisaram o caso de superfícies de ângulo constante em $\mathbb{R}^{3}$ obtendo uma importante relação com a equação de Hamilton-Jacobi e mostrando sua aplicação na configuração de equilíbrio de cristais líquidos. Este trabalho desempenhou um papel fundamental e 
motivacional para o estudo das superfícies de ângulo constante em variedades Riemannianas. No que segue, apresentamos os principais resultados obtidos por Cermelli e Di Scala.

\section{Superfícies de ângulo constante em $\mathbb{R}^{3}$ e cristais líquidos}

Dizemos que uma superfície de $\mathbb{R}^{3}$ é de ângulo constante de acordo com a definição que segue.

Definição 4.1. Seja X um campo de vetores unitário de $\mathbb{R}^{3}$. Uma superfície orientada $M^{2} \subset \mathbb{R}^{3}$ será dita de ângulo constante (com respeito a $X$ ) se seu campo de vetores normal unitário $N$ fizer um ângulo constante $\vartheta$ com $X$.

Desta forma, dada uma superfície $M$, com campo de vetores normais unitários $N$, a condição $M$ ser de ângulo constante com respeito a um campo pré-definido $X$ é

$$
|\langle N, X\rangle|=\cos \vartheta, \quad \vartheta \in[0, \pi / 2] .
$$

Dada uma superfície regular, ao menos localmente, podemos sempre considerar que esta seja uma superfície de nível de uma dada função diferenciável $f: \mathbb{R}^{3} \rightarrow \mathbb{R}$. Isto é, dado um valor regular $c \in \mathbb{R}$ de $f$, consideramos a superfície dada por

$$
M=\left\{x \in \mathbb{R}^{3}: f(x)=c\right\} .
$$

A superfície $M$ assim obtida é diferenciável e possui como campo de vetores normal unitário

$$
N=\frac{\operatorname{grad} f}{\|\operatorname{grad} f\|}
$$

Neste caso a condição (4.1) se torna equivalente a equação quadrática de Hamilton-Jacobi

$$
\left\langle\operatorname{grad} f, A_{\vartheta}(\operatorname{grad} f)\right\rangle=0
$$

sendo $A_{\vartheta}=\left(\cos ^{2} \vartheta\right) \mathrm{Id}-X^{\mathrm{b}} \otimes X$. Definimos a Hamiltoniana associada a esta equação por $H(x, p)=\left\langle p, A_{\vartheta}(x) p\right\rangle$. Em seguida, usando o método característico, constatamos que as solu- 
ções para a equação de Hamilton-Jacobi são as soluções do sistema

$$
\left\{\begin{array}{l}
\dot{x}=\frac{\partial H}{\partial p} \\
\dot{p}=-\frac{\partial H}{\partial x} .
\end{array}\right.
$$

As superfícies de ângulo constante estão relacionadas com o estudo de cristais líquidos em sua fase nemática. Para entender essa relação, primeiro precisamos compreender o que vem a ser um cristal líquido em sua fase nemática. Um cristal líquido, como é descrito em [23], é uma mesofase entre os estados sólido e líquido da matéria. Nesta fase, as moléculas da matéria possuem uma certa organização que intermedeia a completa organização apresentada no estado sólido e a disposição casual do líquido. No caso da fase nemática de um cristal líquido a organização apresentada se trata de uma tendência das moléculas em se alinharem de acordo com uma direção, embora as mesmas não estejam uniformemente agrupadas, possuindo uma distribuição de centros de massa desordenada.

Dessa forma, para descrever um cristal líquido em fase nemática definimos sobre o domínio $\Omega \subset \mathbb{R}^{3}$, que o contém, um campo unitário de direções $X$. Por ser um campo de direções, o campo $X$ pode ser visto como uma aplicação

$$
X: \Omega \rightarrow P^{2}(\mathbb{R})=\mathbb{S}^{2} /\{p,-p\}
$$

Além disso, para que o cristal líquido se encontre em um estado de equilíbrio, o campo $X$ deve ser um ponto crítico do funcional energia

$$
E(X)=\frac{1}{2} \int_{\Omega}\|d X\|^{2} d v
$$

cuja equação de Euler, descrita por Eells e Sampson em [29], é

$$
\Delta X+\|d X\|^{2} X=0
$$

As soluções da equação (4.2) são conhecidas como campos harmônicos.

A conexão com as superfícies de ângulo constante aparece ao considerarmos o bordo $\partial \Omega$ de $\Omega$. No contexto que estamos considerando, $\partial \Omega$ representa uma superfície que separa o cristal líquido 
em sua fase nemática de sua fase isotrópica (aquela onde há ausência de padrões de comportamento de moléculas que não estejam suficientemente próximas, feita exceção pela densidade de partículas, ver [23]). Na natureza, a disposição desta superfície ocorre de forma que, em suas proximidades, a direção na qual as moléculas da fase nemática estão alinhadas forma um ângulo constante com o campo de vetores normal a $\partial \Omega$. Segue daí o interesse no estudo de superfícies de ângulo constante para descrever essas superfícies de separação entre as fases da matéria.

Em [16] P. Cermelli e A. Di Scala consideraram o problema de determinar as superfícies acima citadas em dois casos:

$\left.1^{\circ}\right) X=$ constante.

Neste caso, os autores observam que o fato de $N$ fazer um ângulo constante com uma direção fixa, implica que, ao considerar $N$ como aplicação de $M^{2}$ em $\mathbb{S}^{2}$, sua imagem deve ser uma circunferência. Desta forma concluem que a curvatura Gaussiana da superfície deve ser nula e, como consequência, esta deve ser uma superfície regrada. Em seguida, consideram que a superfície seja localmente um gráfico de uma aplicação $g: D \subset \mathbb{R}^{2} \rightarrow \mathbb{R}$ e $X=\frac{\partial}{\partial z}$. Dessa forma a condição para que $M^{2}$ seja de ângulo constante se torna equivalente à equação eikonal:

$$
|\operatorname{grad} g|=\tan \vartheta
$$

cujas soluções geram superfícies cônicas.

$2^{\circ}$ ) $X$ ortogonal a uma direção constante do espaço.

Para este caso os autores consideram um domínio cilíndrico $\Omega=D \times \mathbb{R}$, com $D \subset \mathbb{R}^{2}$. Então assumem que $X$ seja planar, ou seja considerando uma base ortonormal $\{\mathbf{i}, \mathbf{j}, \mathbf{k}\}$, de forma que a direção $\mathbf{k}$ seja aquela ortogonal a $D$, pode-se escrever o campo $X$ na forma

$$
X=\cos \phi(\mathbf{x}) \mathbf{i}+\operatorname{sen} \phi(\mathbf{x}) \mathbf{j}
$$


sendo $\mathrm{x}$ um ponto de $D$. Com isto, a condição de que $X$ seja um campo harmônico se resume a

$$
\Delta \phi=0
$$

É então considerado o caso em que o campo $X$ possui singularidade ao longo de uma reta vertical paralela a k. Dessa forma as soluções de (4.3) são descritas em função do índice de rotação $k$ de $X$ ao redor da singularidade.

As superfícies obtidas são cilindros com geratriz paralela a k e sobre curvas de $D$. As curvas são descritas como conjunto de nível de uma função diferenciável $f: D \subset \mathbb{R}^{2} \rightarrow \mathbb{R}$, ou seja, denotando por $C$ a curva diretriz, temos

$$
C=\{\mathbf{x} \in D: f(\mathbf{x})=c\}
$$

$\operatorname{com} c \in \mathbb{R}$.

Utilizando coordenadas polares $(\varrho, \alpha)$ as curvas obtidas são classificadas da seguinte forma:

- Se $k \leq 1$, então a curva é dada por

$$
\varrho=\frac{c}{\left|\cos \left(\frac{2-k}{2} \alpha\right)\right|^{\frac{2}{2-k}}} .
$$

- Se $k=2$, então dois subcasos ocorrem:

(I) $\operatorname{se} \vartheta=0$ então a curva é circunferência com $\varrho=$ constante;

(II) $\operatorname{se} \vartheta \neq 0$ então a curva é uma espiral logarítmica com $\varrho=c e^{\alpha \tan \vartheta}$.

- Se $k \geq 3$, então a curva é descrita por

$$
\varrho=c\left|\cos \left(\frac{2-k}{2} \alpha\right)\right|^{\frac{2}{2-k}}
$$

\section{Superfícies de ângulo constante em variedades Riemannianas}

Após o trabalho de Cermielli e Di Scala, o estudo de superfícies de ângulo constante passou a ser considerado em diversas variedades Riemannianas. Em [54] Munteanu e Nistor voltaram a 
considerar as superfícies de ângulo constante no espaço Euclidiano tridimensional, com uma abordagem diferente da feita por Cermelli e Di Scala, obtendo a classificação completa das mesmas. Também foram classificadas as superfícies que fazem ângulo constante com a direção $\mathbb{R}$ nos espaços produtos $\mathbb{S}^{2} \times \mathbb{R}$ e $\mathbb{H}^{2} \times \mathbb{R}$, graças a Dillen-Fastenakels-Van der Veken-Vrancken ( [21]), e Dillen-Munteanu ( [20]), respectivamente. Além disso, subvariedades do tipo hélice foram estudadas no espaço Euclidiano e em espaços produtos de dimensões mais altas (ver [24, 25, 65]).

Ressaltamos que espaços $\mathbb{S}^{2} \times \mathbb{R}$ e $\mathbb{H}^{2} \times \mathbb{R}$ podem ser vistos como dois casos particulares de espaços de Bianchi-Cartan-Vranceanu, que foram descritos na Seção 2.1 e que constituem uma submersão sobre superfícies de curvatura Gaussiana constante. Motivados por este fato e tendo em conta os resultados obtidos (em [18]) por Daniel, descritos na Seção 2.2, em [33] os autores consideraram superfícies em um BCV-espaço na qual o ângulo $\vartheta$ (entre o normal unitário à superfície e o campo tangente as fibras da fibração de Hopf) é constante, dando uma classificação local completa no caso em que este BCV-espaço é o espaço de Heisenberg $\mathbb{H}_{3}$.

Posteriormente, López-Munteanu em [43] definiram e classificaram dois tipos de superfícies de ângulo constante no espaço homogêneo tridimensional $\mathrm{Sol}_{3}$, cujo grupo de isometrias possui dimensão 3. Além disso, Montaldo-Onnis, em [51], caracterizaram as superfícies do tipo hélice na família a um parâmetro das esferas de Berger $\mathbb{S}_{\epsilon}^{3}$, com $\epsilon>0$, provando que, localmente, uma tal superfície é determinada por uma família a um parâmetro de isometrias do ambiente e por uma geodésica de um toro bidimensional na esfera tridimensional.

Neste capítulo apresentamos os resultados que compõem o artigo [52], onde são consideradas as superfícies do tipo hélice no espaço homogêneo tridimensional dado pelo grupo especial linear $\mathrm{SL}(2, \mathbb{R})$ munido com uma família a um parâmetro de métricas $g_{\tau}$ que descreveremos na Seção1.2. Este estudo depende de uma constante $B:=\left(\tau^{2}+1\right) \cos ^{2} \vartheta-1$, onde $\vartheta$ é o ângulo entre o campo normal à superfície e o campo de Hopf de $\operatorname{SL}(2, \mathbb{R})$. Dessa forma, dividiremos nosso estudo de acordo com três possibilidades: $B>0, B=0$ e $B<0$. 


\subsection{Superfícies de ângulo constante em $\mathrm{SL}(2, \mathbb{R})_{\tau}$}

Começamos esta seção dando a definição de superfície de ângulo constante na família a um parâmetro de grupos especiais lineares $\mathrm{SL}(2, \mathbb{R})_{\tau}$ (descrita na Seção 1.2) .

Definição 4.2. Dizemos que uma superfície no grupo especial linear $\mathrm{SL}(2, \mathbb{R})_{\tau}$ é uma superfície do tipo hélice ou uma superfície de ângulo constante se for orientável e o ângulo $\vartheta \in[0, \pi)$ entre seu campo de vetores normal unitário e o campo de vetores de Killing unitário $E_{1}$ (tangente a fibração de Hopf) for constante para todo ponto da superfície.

Seja $M^{2}$ uma superfície de ângulo constante orientada em $\operatorname{SL}(2, \mathbb{R})_{\tau}$ e seja $N$ um campo de vetores normal unitário a $M$. Então, por definição,

$$
\left|g_{\tau}\left(E_{1}, N\right)\right|=\cos \vartheta
$$

para um $\vartheta \in[0, \pi / 2]$ fixo. Observe que $\vartheta \neq 0$. De fato, se $\vartheta$ fosse nulo, $E_{2}$ e $E_{3}$ seriam tangentes à superfície $M^{2}$, o que é absurdo já que a distribuição horizontal da aplicação de Hopf não é integrável. Se $\vartheta=\pi / 2$, teríamos $E_{1}$ sempre tangente a $M$ e, logo, $M$ seria um cilindro de Hopf. Portanto, de agora em diante assumiremos que o ângulo constante $\vartheta \notin\{\pi / 2,0\}$.

Para o estudo das superfícies de ângulo constante em $\mathrm{SL}(2, \mathbb{R})_{\tau}$ nos convém utilizar as técnicas descritas na Seção 2.2. Porém, ressaltamos que devemos verificar a validade destas técnicas para o espaço $\operatorname{SL}(2, \mathbb{R})_{\tau}$, uma vez que o mesmo não compõe os $\mathrm{BCV}$-espaços, não sendo sequer simplesmente conexo.

As fórmulas de Gauss e de Weingarten são dadas por

$$
\begin{aligned}
\nabla^{\tau}{ }_{X} Y & =\nabla_{X} Y+\alpha(X, Y), \\
\nabla^{\tau}{ }_{X} N & =-A(X),
\end{aligned}
$$

onde com $A$ indicamos o operador de forma de $M \operatorname{em~SL}(2, \mathbb{R})_{\tau}$, com $\nabla$ a conexão de Levi-Civita induzida em $M$ e com $\alpha$ a segunda forma fundamental de $M$ em $\operatorname{SL}(2, \mathbb{R})_{\tau}$. Projetando $E_{1}$ no 
plano tangente a $M$ obtemos

$$
E_{1}=T+\cos \vartheta N
$$

onde $T$ denota a sua parte tangente, a qual satisfaz $g_{\tau}(T, T)=\operatorname{sen}^{2} \vartheta$.

Para todo $X \in T M$, temos que

$$
\begin{aligned}
\nabla_{X}^{\tau} E_{1} & =\nabla_{X}^{\tau} T-\cos \vartheta A(X) \\
& =\nabla_{X} T+g_{\tau}(A(X), T) N-\cos \vartheta A(X) .
\end{aligned}
$$

Por outro lado, se $X=\sum X_{i} E_{i}$,

$$
\begin{aligned}
\nabla_{X}^{\tau} E_{1} & =\tau\left(X_{3} E_{2}-X_{2} E_{3}\right)=\tau X \wedge E_{1} \\
& =\tau g_{\tau}(J X, T) N-\tau \cos \vartheta J X
\end{aligned}
$$

onde denotamos por $J X=N \wedge X$ a rotação de ângulo $\pi / 2$ em $T M$. Identificando as componentes tangentes e normais de (4.4) e (4.5) respectivamente, obtemos

$$
\nabla_{X} T=\cos \vartheta(A(X)-\tau J X)
$$

e

$$
g_{\tau}(A(X)-\tau J X, T)=0
$$

Proposição 4.3. As equações de Gauss e Codazzi em $\mathrm{SL}(2, \mathbb{R})_{\tau}$ são, respectivamente,

$$
\begin{gathered}
K=\operatorname{det} A+\tau^{2}-4\left(1+\tau^{2}\right) \cos ^{2} \vartheta \\
\nabla_{X} A Y-\nabla_{Y} A X-A[X, Y]=-4\left(1+\tau^{2}\right) \cos \vartheta\left(g_{\tau}(Y, T) X-g_{\tau}(X, T) Y\right),
\end{gathered}
$$

sendo $X, Y$ campos de vetores tangentes a $M$.

Demonstração. Observamos que as componentes do tensor de curvatura dadas em (1.5) coincidem com as componentes do BCV-espaço $\widetilde{S L}(2, \mathbb{R})$ com $\kappa=-4$, dadas em (2.4). Desta forma, os resultados das Proposições 2.2 e 2.3 se estendem para o espaço $\operatorname{SL}(2, \mathbb{R})_{\tau}$. Consequentemente, a demonstração da Proposição 2.4 se adapta perfeitamente a este caso. 
Lema 4.4. Seja $M^{2}$ uma superfície orientada em $\operatorname{SL}(2, \mathbb{R})_{\tau}$ com ângulo constante $\vartheta$. Então, temos as seguintes propriedades.

(i) Com respeito à base $\{T, J T\}$, a matriz associada ao operador de forma A é

$$
A=\left(\begin{array}{cc}
0 & -\tau \\
-\tau & \lambda
\end{array}\right) \text {, }
$$

para alguma função $\lambda$ em $M$.

(ii) A conexão de Levi-Civita $\nabla$ de $M$ é dada por

$$
\begin{aligned}
& \nabla_{T} T=-2 \tau \cos \vartheta J T, \\
& \nabla_{J T} T=\lambda \cos \vartheta J T, \\
& \nabla_{T} J T=2 \tau \cos \vartheta T, \quad \nabla_{J T} J T=-\lambda \cos \vartheta T .
\end{aligned}
$$

(iii) A curvatura Gaussiana de $M$ é constante e satisfaz

$$
K=-4\left(1+\tau^{2}\right) \cos ^{2} \vartheta
$$

(iv) A função $\lambda$ satisfaz a equação

$$
T \lambda+\lambda^{2} \cos \vartheta+4 B \cos \vartheta=0
$$

onde $B:=\left(\tau^{2}+1\right) \cos ^{2} \vartheta-1$.

Demonstração. O item (i) segue diretamente de (4.7). De (4.6) e usando

$$
g_{\tau}(T, T)=g_{\tau}(J T, J T)=\operatorname{sen}^{2} \vartheta, \quad g_{\tau}(T, J T)=0
$$

obtemos (ii). Da equação de Gauss para $\mathrm{SL}(2, \mathbb{R})_{\tau}$, dada em (4.8), e (i), temos que a curvatura Gaussiana de $M$ é dada por

$$
K=-4\left(1+\tau^{2}\right) \cos ^{2} \vartheta
$$

Finalmente, usando a equação de Codazzi (4.9), $\operatorname{com} X=T, Y=J T$, e usando o item (i) temos

$$
\nabla_{T}(-\tau T+\lambda J T)-\nabla_{J T}(-\tau J T)-A\left(\nabla_{T} J T-\nabla_{J T} T\right)=-4\left(1+\tau^{2}\right) \cos \vartheta\left(-\operatorname{sen}^{2} \vartheta J T\right) .
$$

O resultado segue então de cálculos diretos com o uso de (i) e (ii). 
Observe agora que, sendo $g_{\tau}\left(E_{1}, N\right)=\cos \vartheta$, existe uma função suave $\varphi$ em $M$ tal que

$$
N=\cos \vartheta E_{1}+\operatorname{sen} \vartheta \cos \varphi E_{2}+\operatorname{sen} \vartheta \operatorname{sen} \varphi E_{3} .
$$

Portanto

$$
T=E_{1}-\cos \vartheta N=\operatorname{sen} \vartheta\left[\operatorname{sen} \vartheta E_{1}-\cos \vartheta \cos \varphi E_{2}-\cos \vartheta \operatorname{sen} \varphi E_{3}\right]
$$

e

$$
J T=\operatorname{sen} \vartheta\left(\operatorname{sen} \varphi E_{2}-\cos \varphi E_{3}\right) .
$$

Além disso

$$
\begin{aligned}
A(T) & =-\nabla_{T}^{\tau} N=\left(T \varphi-\tau^{-1}\left(2+\tau^{2}\right) \operatorname{sen}^{2} \vartheta+\tau \cos ^{2} \vartheta\right) J T, \\
A(J T) & =-\nabla_{J T}^{\tau} N=(J T \varphi) J T-\tau T .
\end{aligned}
$$

Comparando (4.12) com o item (i) do Lema 4.4, resulta que

$$
\left\{\begin{array}{l}
J T \varphi=\lambda, \\
T \varphi=-2 \tau^{-1} B .
\end{array}\right.
$$

Observamos que, como

$$
[T, J T]=\left(\nabla_{T} J T-\nabla_{J T} T\right)=\cos \vartheta(2 \tau T-\lambda J T)
$$

a condição de compatibilidade do sistema (4.13),

$$
[T, J T] \varphi=T(J T \varphi)-J T(T \varphi)
$$

é equivalente à equação (4.10).

Agora, consideramos coordenadas locais $(u, v)$ em $M$ de forma que

$$
\partial_{u}=T
$$

Como $\partial_{v}$ é tangente a $M$, podemos escrever

$$
\partial_{v}=a T+b J T
$$


para certas funções definidas por $a=a(u, v)$ e $b=b(u, v)$. De

$$
0=\left[\partial_{u}, \partial_{v}\right]=\left(a_{u}+2 \tau b \cos \vartheta\right) T+\left(b_{u}-b \lambda \cos \vartheta\right) J T
$$

segue

$$
\left\{\begin{array}{l}
a_{u}=-2 \tau b \cos \vartheta \\
b_{u}=b \lambda \cos \vartheta
\end{array}\right.
$$

Além disso, a equação (4.10) do Lema 4.4 pode ser rescrita como

$$
\lambda_{u}+\cos \vartheta \lambda^{2}+4 B \cos \vartheta=0 .
$$

Dependendo do valor de $B$ a integração de (4.17) gera as possibilidades que seguem.

(i) $\mathrm{Se} B=0$,

$$
\lambda(u, v)=\frac{1}{u \cos \vartheta+\eta(v)},
$$

para alguma função suave $\eta$ dependendo de $v$. Então, a solução do sistema (4.16) é dada por

$$
\left\{\begin{array}{l}
a(u, v)=-\tau u \cos \vartheta(u \cos \vartheta+2 \eta(v)), \\
b(u, v)=u \cos \vartheta+\eta(v) .
\end{array}\right.
$$

(ii) Se $B>0$,

$$
\lambda(u, v)=2 \sqrt{B} \tan (\eta(v)-2 \cos \vartheta \sqrt{B} u),
$$

para alguma função suave $\eta$ dependendo de $v$. Resolvendo o sistema (4.16), obtemos

$$
\left\{\begin{array}{l}
a(u, v)=\frac{\tau}{\sqrt{B}} \operatorname{sen}(\eta(v)-2 \cos \vartheta \sqrt{B} u), \\
b(u, v)=\cos (\eta(v)-2 \cos \vartheta \sqrt{B} u)
\end{array}\right.
$$

(iii) $\operatorname{Se} B<0$,

$$
\lambda(u, v)=2 \sqrt{-B} \tanh (\eta(v)+2 \cos \vartheta \sqrt{-B} u),
$$

para alguma função suave $\eta$ dependendo de $v$. Resolvendo o sistema (4.16), temos

$$
\left\{\begin{array}{l}
a(u, v)=-\frac{\tau}{\sqrt{-B}} \operatorname{senh}(\eta(v)+2 \cos \vartheta \sqrt{-B} u), \\
b(u, v)=\cosh (\eta(v)+2 \cos \vartheta \sqrt{-B} u) .
\end{array}\right.
$$


Ainda, no caso (i) o sistema (4.13) se torna

$$
\left\{\begin{array}{l}
\varphi_{u}=0, \\
\varphi_{v}=1,
\end{array}\right.
$$

e portanto $\varphi(u, v)=v+c, c \in \mathbb{R}$. Nos casos (ii) e (iii), o sistema (4.13) se torna

$$
\left\{\begin{array}{l}
\varphi_{u}=-2 \tau^{-1} B \\
\varphi_{v}=0
\end{array}\right.
$$

para o qual a solução geral é dada por

$$
\varphi(u, v)=-2 \tau^{-1} B u+c
$$

onde $c$ é uma constante real.

Com respeito às coordenadas locais $(u, v)$ que estamos considerando, temos a seguinte caracterização para o vetor posição de uma superfície de ângulo constante em $\operatorname{SL}(2, \mathbb{R})_{\tau}$.

Proposição 4.5. Seja $M^{2}$ uma superfície de ângulo constante $\vartheta$ em $\mathrm{SL}(2, \mathbb{R})_{\tau} \subset \mathbb{R}_{2}^{4}$. Então, com respeito às coordenadas locais $(u, v)$ em $M$ definidas em (4.14), o vetor posição $F$ de $M^{2}$ em $\mathbb{R}_{2}^{4}$ satisfaz as seguintes equações:

(a) se $B=0$,

$$
\frac{\partial^{2} F}{\partial u^{2}}=0
$$

(b) se $B \neq 0$,

$$
\frac{\partial^{4} F}{\partial u^{4}}+\left(\tilde{b}^{2}-2 \tilde{a}\right) \frac{\partial^{2} F}{\partial u^{2}}+\tilde{a}^{2} F=0
$$

onde

$$
\tilde{a}=-\tau^{-2} \operatorname{sen}^{2} \vartheta B, \quad \tilde{b}=-2 \tau^{-1} B
$$

Demonstração. Seja $M^{2}$ uma superfície de ângulo constante e seja $F$ o vetor posição de $M^{2}$ em $\mathbb{R}_{2}^{4}$. Então, com respeito às coordenadas locais $(u, v)$ em $M$ definidas em (4.14), podemos escrever 
$F(u, v)=\left(F_{1}(u, v), \ldots, F_{4}(u, v)\right)$. Por definição, considerando (4.11), temos que

$$
\begin{aligned}
F_{u} & =\left(\partial_{u} F_{1}, \partial_{u} F_{2}, \partial_{u} F_{3}, \partial_{u} F_{4}\right)=T \\
& =\operatorname{sen} \vartheta\left[\operatorname{sen} \vartheta E_{1 \mid F(u, v)}-\cos \vartheta \cos \varphi E_{2 \mid F(u, v)}-\cos \vartheta \operatorname{sen} \varphi E_{3 \mid F(u, v)}\right] .
\end{aligned}
$$

Usando as expressões de $E_{1}, E_{2}$ e $E_{3}$ com respeito aos campos coordenados de $\mathbb{R}_{2}^{4}$, a última equação implica em

$$
\left\{\begin{array}{l}
\partial_{u} F_{1}=\operatorname{sen} \vartheta\left(\tau^{-1} \operatorname{sen} \vartheta F_{2}-\cos \vartheta \cos \varphi F_{4}-\cos \vartheta \operatorname{sen} \varphi F_{3}\right), \\
\partial_{u} F_{2}=-\operatorname{sen} \vartheta\left(\tau^{-1} \operatorname{sen} \vartheta F_{1}+\cos \vartheta \cos \varphi F_{3}-\cos \vartheta \operatorname{sen} \varphi F_{4}\right), \\
\partial_{u} F_{3}=\operatorname{sen} \vartheta\left(\tau^{-1} \operatorname{sen} \vartheta F_{4}-\cos \vartheta \cos \varphi F_{2}-\cos \vartheta \operatorname{sen} \varphi F_{1}\right), \\
\partial_{u} F_{4}=-\operatorname{sen} \vartheta\left(\tau^{-1} \operatorname{sen} \vartheta F_{3}+\cos \vartheta \cos \varphi F_{1}-\cos \vartheta \operatorname{sen} \varphi F_{2}\right) .
\end{array}\right.
$$

Portanto, se $B=0$, derivando (4.23) com respeito a $u$ e usando (4.18), obtemos que $F_{u u}=0$.

Se $B \neq 0$, derivando (4.23) com respeito a $u$ e usando (4.19), encontramos duas constantes $\tilde{a}$ e $\tilde{b}$ tais que

$$
\left\{\begin{array}{l}
\left(F_{1}\right)_{u u}=\tilde{a} F_{1}+\tilde{b}\left(F_{2}\right)_{u} \\
\left(F_{2}\right)_{u u}=\tilde{a} F_{2}-\tilde{b}\left(F_{1}\right)_{u} \\
\left(F_{3}\right)_{u u}=\tilde{a} F_{3}+\tilde{b}\left(F_{4}\right)_{u} \\
\left(F_{4}\right)_{u u}=\tilde{a} F_{4}-\tilde{b}\left(F_{3}\right)_{u}
\end{array}\right.
$$

onde

$$
\tilde{a}=\frac{\tau^{-1} \operatorname{sen}^{2} \vartheta}{2} \varphi_{u}=-\tau^{-2} \operatorname{sen}^{2} \vartheta B, \quad \tilde{b}=\varphi_{u} .
$$

Finalmente, derivando duas vezes (4.24) com respeito a $u$ e usando (4.23) e (4.24) obtemos a equação desejada (4.21).

Observação 4.1. Como $\langle F, F\rangle=1$, usando (4.21), (4.23) e (4.24), concluímos que $F(u, v)$ e suas derivadas devem satisfazer as relações:

$$
\begin{array}{lll}
\langle F, F\rangle=1, \quad\left\langle F_{u}, F_{u}\right\rangle=\tilde{a}, & \left\langle F, F_{u}\right\rangle=0, \\
\left\langle F_{u}, F_{u u}\right\rangle=0, \quad\left\langle F_{u u}, F_{u u}\right\rangle=D, & \left\langle F, F_{u u}\right\rangle=-\tilde{a}, \\
\left\langle F_{u}, F_{\text {uuu }}\right\rangle=-D, & \left\langle F_{\text {uu }}, F_{\text {uuu }}\right\rangle=0, & \left\langle F, F_{\text {uuu }}\right\rangle=0, \\
\left\langle F_{\text {uuu }}, F_{\text {uuu }}\right\rangle=E, &
\end{array}
$$


onde

$$
D=\tilde{a} \tilde{b}^{2}-3 \tilde{a}^{2}, \quad E=\left(\tilde{b}^{2}-2 \tilde{a}\right) D-\tilde{a}^{3}
$$

Mais ainda, como

$$
J_{1} F(u, v)=X_{1 \mid F(u, v)}=-\tau E_{1 \mid F(u, v)}=-\tau\left(F_{u}+\cos \vartheta N\right),
$$

usando (4.21)-4.25), obtemos as seguintes identidades

$$
\begin{aligned}
& \left\langle J_{1} F, F_{u}\right\rangle=-\tau^{-1} \operatorname{sen}^{2} \vartheta, \\
& \left\langle J_{1} F, F_{u u}\right\rangle=0, \\
& \left\langle F_{u}, J_{1} F_{u u}\right\rangle=\tilde{a}\left(\tilde{b}-\tau^{-1} \operatorname{sen}^{2} \vartheta\right):=I, \\
& \left\langle J_{1} F_{u}, F_{\text {uuu }}\right\rangle=0, \\
& \left\langle J_{1} F_{u}, F_{\text {uu }}\right\rangle+\left\langle J_{1} F, F_{\text {uuu }}\right\rangle=0, \\
& \left\langle J_{1} F_{u u}, F_{\text {uuи }}\right\rangle+\left\langle J_{1} F_{u}, F_{\text {uuиu }}\right\rangle=0 .
\end{aligned}
$$

Usando a Observação 4.1 podemos provar a seguinte proposição que dá condições sobre as quais uma imersão define uma superfície de ângulo constante.

Proposição 4.6. Seja $F: \Omega \rightarrow \operatorname{SL}(2, \mathbb{R})_{\tau} \subset \mathbb{R}_{2}^{4}$ uma imersão de um conjunto aberto $\Omega \subset \mathbb{R}^{2}$, com $F(\Omega)$ superfície orientada e $(u, v)$ coordenadas locais tais que a projeção de $E_{1}=-\tau^{-1} J_{1} F$ no espaço tangente de $F(\Omega) \subset \mathrm{SL}(2, \mathbb{R})_{\tau}$ seja $F_{u}$. Então $F(\Omega) \subset \mathrm{SL}(2, \mathbb{R})_{\tau}$ descreve uma superfície de ângulo constante $\vartheta$ se, e somente se,

$$
g_{\tau}\left(F_{u}, F_{u}\right)=g_{\tau}\left(E_{1}, F_{u}\right)=\operatorname{sen}^{2} \vartheta
$$

$e$

$$
g_{\tau}\left(F_{v}, E_{1}\right)-g_{\tau}\left(F_{u}, F_{v}\right)=0 .
$$

Demonstração. Suponha que $F$ seja uma superfície de ângulo constante $\vartheta$. Então

$$
\begin{aligned}
g_{\tau}\left(F_{u}, F_{u}\right) & =-\left\langle F_{u}, F_{u}\right\rangle+\left(1+\tau^{2}\right)\left\langle F_{u}, J_{1} F\right\rangle^{2} \\
& =\tau^{-2} \operatorname{sen}^{2} \vartheta B+\left(1+\tau^{2}\right)\left(\tau^{-2} \operatorname{sen}^{4} \vartheta\right) \\
& =\operatorname{sen}^{2} \vartheta .
\end{aligned}
$$


Analogamente

$$
\begin{aligned}
g_{\tau}\left(E_{1}, F_{u}\right) & =\tau^{-1}\left\langle J_{1} F, F_{u}\right\rangle-\tau^{-1}\left(1+\tau^{2}\right)\left\langle J_{1} F, F_{u}\right\rangle\left\langle J_{1} F, J_{1} F\right\rangle \\
& =\tau^{-1}\left\langle J_{1} F, F_{u}\right\rangle\left[1-\left(1+\tau^{2}\right)\right]=\operatorname{sen}^{2} \vartheta
\end{aligned}
$$

Por fim, usando (4.15), temos

$$
\begin{aligned}
g_{\tau}\left(F_{v}, E_{1}\right)-g_{\tau}\left(F_{u}, F_{v}\right) & =-\frac{a}{\tau} g_{\tau}\left(F_{u}, J_{1} F\right)-\frac{b}{\tau} g_{\tau}\left(J_{1} F_{u}, J_{1} F\right)-a g_{\tau}\left(F_{u}, F_{u}\right)-b g_{\tau}\left(J_{1} F_{u}, F_{u}\right) \\
& =a \operatorname{sen}^{2} \vartheta-0-a \operatorname{sen}^{2} \vartheta-0=0 .
\end{aligned}
$$

Para a volta, seja

$$
T_{2}:=F_{v}-\frac{g_{\tau}\left(F_{v}, F_{u}\right) F_{u}}{g_{\tau}\left(F_{u}, F_{u}\right)}
$$

Então, se denotamos por $N$ o campo unitário normal à superfície $F(\Omega)$, temos que $\left\{F_{u}, T_{2}, N\right\}$ é uma base ortogonal do espaço tangente de $\mathrm{SL}(2, \mathbb{R})_{\tau}$ ao longo da superfície $F(\Omega)$. Agora, usando (4.28), obtemos $g_{\tau}\left(E_{1}, T_{2}\right)=0$, e logo $E_{1}=a F_{u}+c N$. Além disso, usando (4.27) e $g_{\tau}\left(E_{1}, F_{u}\right)=a g_{\tau}\left(F_{u}, F_{u}\right)$, concluímos que $a=1$. Finalmente,

$$
1=g_{\tau}\left(E_{1}, E_{1}\right)=g_{\tau}\left(F_{u}+c N, F_{u}+c N\right)=\operatorname{sen}^{2} \vartheta+c^{2}
$$

o que implica que $c^{2}=\cos ^{2} \vartheta$. Portanto

$$
g_{\tau}\left(E_{1}, N\right)=g_{\tau}\left(F_{u}+\cos \vartheta N, N\right)=\cos \vartheta
$$

o que termina a prova.

Antes de passar ao estudo dos três casos dependendo da constante $B$ ser nula, positiva ou negativa, fazemos a seguinte

Observação 4.2. Consideramos uma família a um parâmetro $A(v), v \in(a, b) \subset \mathbb{R}$, que consiste em matrizes $4 \times 4$ ortogonais indefinidas que comutam (respectivamente anti-comutam) com $J_{1}$. Para descrever explicitamente a família $A(v)$, usaremos as duas estruturas de produto de $\mathbb{R}_{2}^{4}$ dadas 
por

$$
J_{2}=\left(\begin{array}{cccc}
0 & 0 & 0 & 1 \\
0 & 0 & 1 & 0 \\
0 & 1 & 0 & 0 \\
1 & 0 & 0 & 0
\end{array}\right), \quad J_{3}=\left(\begin{array}{cccc}
0 & 0 & 1 & 0 \\
0 & 0 & 0 & -1 \\
1 & 0 & 0 & 0 \\
0 & -1 & 0 & 0
\end{array}\right) .
$$

Como $A(v)$ é uma matriz ortogonal indefinida, sua primeira linha deve ser um vetor unitário $\mathbf{r}_{1}(v)$ de $\mathbb{R}_{2}^{4}$ para todo $v \in(a, b)$. Portando, sem perda de generalidade, tomamos

$\mathbf{r}_{1}(v)=\left(\cosh \xi_{1}(v) \cos \xi_{2}(v),-\cosh \xi_{1}(v) \operatorname{sen} \xi_{2}(v), \operatorname{senh} \xi_{1}(v) \cos \xi_{3}(v),-\operatorname{senh} \xi_{1}(v) \operatorname{sen} \xi_{3}(v)\right)$

sendo $\xi_{1}, \xi_{2}$ e $\xi_{3}$ funções reais definidas em $(a, b)$. Como $A(v)$ comuta (anti-comuta, respectivamente) com $J_{1}$, a segunda linha de $A(v)$ deve ser $\mathbf{r}_{2}(v)= \pm J_{1} \mathbf{r}_{1}(v)$. Dessa forma, os vetores $\left\{\mathbf{r}_{1}, J_{1} \mathbf{r}_{1}, J_{2} \mathbf{r}_{1}, J_{3} \mathbf{r}_{1}\right\}$ formam uma base pseudo-ortonormal de $\mathbb{R}_{2}^{4}$, portanto a terceira linha $\mathbf{r}_{3}(v)$ de $A(v)$ será uma combinação linear destes. Como $\mathbf{r}_{3}(v)$ é unitário e ortogonal a ambos $\mathbf{r}_{1}(v) e$ $J_{1} \mathbf{r}_{1}(v)$, existe uma função $\xi(v)$ tal que

$$
\mathbf{r}_{3}(v)=\cos \xi(v) J_{2} \mathbf{r}_{1}(v)+\operatorname{sen} \xi(v) J_{3} \mathbf{r}_{1}(v) .
$$

Por fim, a quarta linha de $A(v)$ será $\mathbf{r}_{4}(v)= \pm J_{1} \mathbf{r}_{3}(v)=\mp \cos \xi(v) J_{3} \mathbf{r}_{1}(v) \pm \operatorname{sen} \xi(v) J_{2} \mathbf{r}_{1}(v)$. Isso significa que qualquer família a um parâmetro $A(v)$ de matrizes $4 \times 4$ ortogonais indefinidas que comutam (anti-comutam, respectivamente) com $J_{1}$ podem ser descritas por quatro funções $\xi_{1}, \xi_{2}, \xi_{3}$ e $\xi$ da forma

$$
A\left(\xi, \xi_{1}, \xi_{2}, \xi_{3}\right)(v)=\left(\begin{array}{c}
\mathbf{r}_{1}(v) \\
\pm J_{1} \mathbf{r}_{1}(v) \\
\cos \xi(v) J_{2} \mathbf{r}_{1}(v)+\operatorname{sen} \xi(v) J_{3} \mathbf{r}_{1}(v) \\
\mp \cos \xi(v) J_{3} \mathbf{r}_{1}(v) \pm \operatorname{sen} \xi(v) J_{2} \mathbf{r}_{1}(v)
\end{array}\right)
$$




\subsection{O caso $B=0$}

Teorema 4.7. Seja $M^{2}$ uma superfície de ângulo constante $\vartheta$ em $\operatorname{SL}(2, \mathbb{R})_{\tau} \subset \mathbb{R}_{2}^{4}$ tal que $B=0$. Então,

$$
\cos \vartheta=\frac{1}{\sqrt{1+\tau^{2}}}
$$

e, localmente, o vetor posição de $M^{2}$ em $\mathbb{R}_{2}^{4}$, com respeito às coordenadas locais $(u, v)$ em $M$ definidas em (4.14), é dado por

$$
F(u, v)=A(v)\left(1,-\frac{\tau u}{1+\tau^{2}}, \frac{\tau u}{1+\tau^{2}}, 0\right)
$$

onde $A(v)=A\left(\xi, \xi_{1}, \xi_{2}, \xi_{3}\right)(v)$ é uma família a um parâmetro de matrizes $4 \times 4$ ortogonais indefinidas que comutam com $J_{1}$, como descrito em (4.29), com

$$
\begin{aligned}
& {\left[-\xi^{\prime}(v)+\xi_{2}^{\prime}(v)+\xi_{3}^{\prime}(v)\right] \operatorname{sen}\left(\xi_{2}(v)-\xi_{3}(v)\right) \operatorname{senh}\left(2 \xi_{1}(v)\right)-} \\
& 2\left(\xi^{\prime}(v)-\xi_{3}^{\prime}(v)\right) \operatorname{senh}^{2} \xi_{1}(v)+2\left[\xi_{1}^{\prime}(v) \cos \left(\xi_{2}(v)-\xi_{3}(v)\right)+\xi_{2}^{\prime}(v) \cosh ^{2} \xi_{1}(v)\right]=0 .
\end{aligned}
$$

Reciprocamente, uma parametrização

$$
F(u, v)=A(v)\left(1,-\frac{\tau u}{1+\tau^{2}}, \frac{\tau u}{1+\tau^{2}}, 0\right),
$$

com $A(v)$ como acima, define uma superfície de ângulo constante no grupo especial linear com ângulo $\vartheta=\arccos \frac{1}{\sqrt{1+\tau^{2}}}$.

Demonstração. Como $B=0$ temos imediatamente que $\cos ^{2} \vartheta=1 /\left(1+\tau^{2}\right)$. Integrando (4.20), obtemos que

$$
F(u, v)=h^{1}(v)+u h^{2}(v),
$$

onde $h^{i}(v), i=1,2$, são campos de vetores em $\mathbb{R}_{2}^{4}$, dependendo apenas de $v$.

Avaliando em $(0, v)$ as identidades:

$$
\begin{aligned}
& \langle F, F\rangle=1, \quad\left\langle F_{u}, F_{u}\right\rangle=0, \\
& \left\langle F, F_{u}\right\rangle=0, \quad\left\langle J_{1} F, F_{u}\right\rangle=-\tau^{-1} \operatorname{sen}^{2} \vartheta=-\frac{\tau}{1+\tau^{2}},
\end{aligned}
$$


resulta que

$$
\begin{aligned}
& \left\langle h^{1}(v), h^{1}(v)\right\rangle=1, \quad\left\langle h^{1}(v), h^{2}(v)\right\rangle=0, \\
& \left\langle h^{2}(v), h^{2}(v)\right\rangle=0, \quad\left\langle J_{1} h^{1}(v), h^{2}(v)\right\rangle=-\frac{\tau}{1+\tau^{2}} .
\end{aligned}
$$

Além disso, usando (4.23) em $(0, v)$ e $\operatorname{sen}^{2} \vartheta=\tau^{2}\left(1+\tau^{2}\right)^{-1}$, temos que

$$
h^{2}(v)=-\frac{\tau}{1+\tau^{2}}\left(J_{1} h^{1}(v)-h^{3}(v)\right)
$$

onde, em virtude de (4.32), sabemos que $h^{3}(v)$ é um campo de vetores em $\mathbb{R}_{2}^{4}$ satisfazendo

$$
\left\langle h^{3}(v), h^{3}(v)\right\rangle=-1, \quad\left\langle h^{1}(v), h^{3}(v)\right\rangle=0, \quad\left\langle J_{1} h^{1}(v), h^{3}(v)\right\rangle=0 .
$$

Consequentemente, se fixarmos a base ortonormal $\left\{\hat{E}_{i}\right\}_{i=1}^{4}$ de $\mathbb{R}_{2}^{4}$ dada por

$$
\hat{E}_{1}=(1,0,0,0), \quad \hat{E}_{2}=(0,1,0,0), \quad \hat{E}_{3}=(0,0,1,0), \quad \hat{E}_{4}=(0,0,0,1),
$$

deve existir uma família a um parâmetro de matrizes $A(v) \in \mathrm{O}_{2}(4)$, com $J_{1} A(v)=A(v) J_{1}$, de forma que

$$
h^{1}(v)=A(v) \hat{E}_{1}, \quad J_{1} h^{1}(v)=A(v) \hat{E}_{2}, \quad h^{3}(v)=A(v) \hat{E}_{3}, \quad J_{1} h^{3}(v)=A(v) \hat{E}_{4} .
$$

Então (4.31) se torna

$$
F(u, v)=h^{1}(v)-\frac{\tau u}{1+\tau^{2}}\left(J_{1} h^{1}(v)-h^{3}(v)\right)=A(v)\left(1,-\frac{\tau u}{1+\tau^{2}}, \frac{\tau u}{1+\tau^{2}}, 0\right) .
$$

Observamos que $F(u, v)$ como acima escrita, garante que (4.28) seja equivalente a $\left\langle F_{u}, F_{v}\right\rangle=0$. De fato, usando (1.3), podemos reescrever (4.28) na forma

$$
-\left\langle F_{v}, E_{1}\right\rangle+\left(1+\tau^{2}\right)\left\langle F_{v}, X_{1}\right\rangle\left\langle E_{1}, X_{1}\right\rangle+\left\langle F_{u}, F_{v}\right\rangle-\left(1+\tau^{2}\right)\left\langle F_{u}, X_{1}\right\rangle\left\langle F_{v}, X_{1}\right\rangle=0,
$$

e então, usando (4.33) para calcular $\left\langle F_{u}, X_{1}\right\rangle$ e substituindo $E_{1}=\tau^{-1} X_{1}$, obtemos $\left\langle F_{u}, F_{v}\right\rangle=0$.

Finalmente, analisemos a família a um parâmetro $A(v)$. De acordo com 44.29), $A(v)$ depende de quatro funções $\xi_{1}(v), \xi_{2}(v), \xi_{3}(v)$ e $\xi(v)$. Como neste caso a condição (4.28) se reduz a $\left\langle F_{u}, F_{v}\right\rangle=0$, que é equivalente à equação (4.30), concluímos esta parte da demonstração. 
Para a volta, seja

$$
F(u, v)=A(v)\left(1,-\frac{\tau u}{1+\tau^{2}}, \frac{\tau u}{1+\tau^{2}}, 0\right),
$$

uma parametrização onde $A(v)=A\left(\xi(v), \xi_{1}(v), \xi_{2}(v), \xi_{3}(v)\right)$ é uma família a um parâmetro de matrizes ortogonais com funções $\xi(v), \xi_{1}(v), \xi_{2}(v), \xi_{3}(v)$ satisfazendo (4.30). Como $A(v)$ satisfaz (4.30), F satisfaz (4.28), e portanto, em virtude da Proposição 4.6, devemos mostrar apenas que (4.27) é satisfeita para algum ângulo constante $\vartheta$. Para este fim, seja

$$
\gamma(u)=\left(1,-\frac{\tau u}{1+\tau^{2}}, \frac{\tau u}{1+\tau^{2}}, 0\right) .
$$

Usando (1.3) e levando em consideração que $A(v)$ comuta com $J_{1}$, obtemos

$$
\begin{aligned}
g_{\tau}\left(F_{u}, F_{u}\right) & =-\left\langle A(v) \gamma^{\prime}(u), A(v) \gamma^{\prime}(u)\right\rangle+\left(1+\tau^{2}\right)\left\langle A(v) \gamma^{\prime}(u), J_{1} A(v) \gamma(u)\right\rangle^{2} \\
& =\left(1+\tau^{2}\right)\left\langle\gamma^{\prime}(u), J_{1} \gamma(u)\right\rangle^{2}=\frac{\tau^{2}}{1+\tau^{2}}
\end{aligned}
$$

e podemos escolher $\vartheta$ tal que $\tau^{2} /\left(1+\tau^{2}\right)=\operatorname{sen}^{2} \vartheta$. Analogamente,

$$
\begin{aligned}
g_{\tau}\left(E_{1}, F_{u}\right) & =-\left\langle E_{1}, F_{u}\right\rangle+\left(1+\tau^{2}\right)\left\langle E_{1}, J_{1} F\right\rangle\left\langle F_{u}, J_{1} F\right\rangle \\
& =\frac{\left\langle F_{u}, J_{1} F\right\rangle}{\tau}\left[1-\left(1+\tau^{2}\right)\right] \\
& =(-\tau)\left\langle\gamma^{\prime}(u), J_{1} \gamma(u)\right\rangle=\frac{\tau^{2}}{1+\tau^{2}} .
\end{aligned}
$$

\subsection{O caso $B>0$}

Quando $B>0$, a integração de (4.21) fornece a seguinte

Proposição 4.8. Seja $M^{2}$ uma superfície de ângulo constante em $\operatorname{SL}(2, \mathbb{R})_{\tau}$ com ângulo $\vartheta$ tal que $B>0$. Então, com respeito às coordenadas locais $(u, v)$ de $M$ definidas em (4.14), o vetor posição F de $M^{2}$ em $\mathbb{R}_{2}^{4}$ é dado por

$$
F(u, v)=\cos \left(\alpha_{1} u\right) g^{1}(v)+\operatorname{sen}\left(\alpha_{1} u\right) g^{2}(v)+\cos \left(\alpha_{2} u\right) g^{3}(v)+\operatorname{sen}\left(\alpha_{2} u\right) g^{4}(v),
$$


onde

$$
\alpha_{1,2}=\frac{1}{\tau}(\tau \sqrt{B} \cos \vartheta \pm B)
$$

são constantes reais positivas, enquanto que $g^{i}(v), i \in\{1, \ldots, 4\}$, são campos de vetores ortogonais entre si em $\mathbb{R}_{2}^{4}$, dependendo apenas de $v$, tais que

$$
\begin{aligned}
& g_{11}=\left\langle g^{1}(v), g^{1}(v)\right\rangle=g_{22}=\left\langle g^{2}(v), g^{2}(v)\right\rangle=-\frac{\tau}{2 B} \alpha_{2}, \\
& g_{33}=\left\langle g^{3}(v), g^{3}(v)\right\rangle=g_{44}=\left\langle g^{4}(v), g^{4}(v)\right\rangle=\frac{\tau}{2 B} \alpha_{1} .
\end{aligned}
$$

Demonstração. Primeiro, uma integração direta de (4.21), confere a solução

$$
F(u, v)=\cos \left(\alpha_{1} u\right) g^{1}(v)+\operatorname{sen}\left(\alpha_{1} u\right) g^{2}(v)+\cos \left(\alpha_{2} u\right) g^{3}(v)+\operatorname{sen}\left(\alpha_{2} u\right) g^{4}(v)
$$

onde

$$
\alpha_{1,2}=\sqrt{\frac{\tilde{b}^{2}-2 \tilde{a} \pm \sqrt{\tilde{b}^{4}-4 \tilde{a} \tilde{b}^{2}}}{2}}
$$

são duas constantes, enquanto que $g^{i}(v), i \in\{1, \ldots, 4\}$, são campos de vetores em $\mathbb{R}_{2}^{4}$ que dependem apenas de $v$. Agora, levando em consideração os valores de $\tilde{a}$ e $\tilde{b}$ dados em (4.22), obtemos

$$
\alpha_{1,2}=\frac{1}{\tau}(\tau \sqrt{B} \cos \vartheta \pm B)
$$

Fazendo $g_{i j}(v)=\left\langle g^{i}(v), g^{j}(v)\right\rangle$, e avaliando as relações (4.25) em $(0, v)$, obtemos:

$$
\begin{gathered}
g_{11}+g_{33}+2 g_{13}=1, \\
\alpha_{1}^{2} g_{22}+\alpha_{2}^{2} g_{44}+2 \alpha_{1} \alpha_{2} g_{24}=\tilde{a}, \\
\alpha_{1} g_{12}+\alpha_{2} g_{14}+\alpha_{1} g_{23}+\alpha_{2} g_{34}=0, \\
\alpha_{1}^{3} g_{12}+\alpha_{1} \alpha_{2}^{2} g_{23}+\alpha_{1}^{2} \alpha_{2} g_{14}+\alpha_{2}^{3} g_{34}=0, \\
\alpha_{1}^{4} g_{11}+\alpha_{2}^{4} g_{33}+2 \alpha_{1}^{2} \alpha_{2}^{2} g_{13}=D, \\
\alpha_{1}^{2} g_{11}+\alpha_{2}^{2} g_{33}+\left(\alpha_{1}^{2}+\alpha_{2}^{2}\right) g_{13}=\tilde{a}, \\
\alpha_{1}^{4} g_{22}+\alpha_{1}^{3} \alpha_{2} g_{24}+\alpha_{1} \alpha_{2}^{3} g_{24}+\alpha_{2}^{4} g_{44}=D,
\end{gathered}
$$




$$
\begin{gathered}
\alpha_{1}^{5} g_{12}+\alpha_{1}^{3} \alpha_{2}^{2} g_{23}+\alpha_{1}^{2} \alpha_{2}^{3} g_{14}+\alpha_{2}^{5} g_{34}=0, \\
\alpha_{1}^{3} g_{12}+\alpha_{1}^{3} g_{23}+\alpha_{2}^{3} g_{14}+\alpha_{2}^{3} g_{34}=0, \\
\alpha_{1}^{6} g_{22}+\alpha_{2}^{6} g_{44}+2 \alpha_{1}^{3} \alpha_{2}^{3} g_{24}=E .
\end{gathered}
$$

De (4.38), 4.39), (4.43), (4.44), segue que

$$
g_{12}=g_{14}=g_{23}=g_{34}=0 \text {. }
$$

Além disso, de (4.36), 4.40) e 4.41), obtemos

$$
g_{11}=\frac{\tau^{2}\left(D+\alpha_{2}^{4}\right)+2 B \operatorname{sen}^{2} \vartheta \alpha_{2}^{2}}{\tau^{2}\left(\alpha_{1}^{2}-\alpha_{2}^{2}\right)^{2}}, \quad g_{13}=0, \quad g_{33}=\frac{\tau^{2}\left(D+\alpha_{1}^{4}\right)+2 B \operatorname{sen}^{2} \vartheta \alpha_{1}^{2}}{\tau^{2}\left(\alpha_{1}^{2}-\alpha_{2}^{2}\right)^{2}} .
$$

Finalmente, usando 4.37, 4.42) e 4.45), obtemos

$$
g_{22}=\frac{\tau^{2}\left(E-2 D \alpha_{2}^{2}\right)-B \operatorname{sen}^{2} \vartheta \alpha_{2}^{4}}{\tau^{2} \alpha_{1}^{2}\left(\alpha_{1}^{2}-\alpha_{2}^{2}\right)^{2}}, \quad g_{24}=0, \quad g_{44}=\frac{\tau^{2}\left(E-2 D \alpha_{1}^{2}\right)-B \operatorname{sen}^{2} \vartheta \alpha_{1}^{4}}{\tau^{2} \alpha_{2}^{2}\left(\alpha_{1}^{2}-\alpha_{2}^{2}\right)^{2}} .
$$

Observamos que

$$
g_{11}=g_{22}=\frac{\sqrt{B}-\tau \cos \vartheta}{2 \sqrt{B}}<0, \quad g_{33}=g_{44}=\frac{\sqrt{B}+\tau \cos \vartheta}{2 \sqrt{B}}>0 .
$$

Portanto, levando em conta (4.35), obtemos as expressões (4.34).

Agora estamos prontos para anunciar o resultado principal desta seção.

Teorema 4.9. Seja $M^{2}$ uma superfície de ângulo constante em $\operatorname{SL}(2, \mathbb{R})_{\tau} \subset \mathbb{R}_{2}^{4}$, com ângulo $\vartheta \neq \pi / 2$ tal que $B>0$. Então, localmente, o vetor posição de $M^{2}$ em $\mathbb{R}_{2}^{4}$, com respeito às coordenadas locais $(u, v)$ de $M$ definidas em (4.14), é dado por

$$
F(u, v)=A(v) \gamma(u)
$$

onde

$$
\gamma(u)=\left(\sqrt{g_{33}} \cos \left(\alpha_{2} u\right),-\sqrt{g_{33}} \operatorname{sen}\left(\alpha_{2} u\right), \sqrt{-g_{11}} \cos \left(\alpha_{1} u\right), \sqrt{-g_{11}} \operatorname{sen}\left(\alpha_{1} u\right)\right)
$$


é uma curva em $\mathrm{SL}(2, \mathbb{R})_{\tau}, g_{11}, g_{33}, \alpha_{1}, \alpha_{2}$ são as quatro constantes dadas na Proposição 4.8 ] $A(v)=A\left(\xi, \xi_{1}, \xi_{2}, \xi_{3}\right)(v)$ é uma família a um parâmetro de matrizes $4 \times 4$ ortogonais indefinidas que comutam com $J_{1}$, como descrito em 4.29), com $\xi=$ constante $e$

$$
\cosh ^{2}\left(\xi_{1}(v)\right) \xi_{2}^{\prime}(v)+\operatorname{senh}^{2}\left(\xi_{1}(v)\right) \xi_{3}^{\prime}(v)=0
$$

Reciprocamente, uma parametrização $F(u, v)=A(v) \gamma(u)$, com $\gamma(u)$ e A(v) como acima, define uma superfície de ângulo constante em $\mathrm{SL}(2, \mathbb{R})_{\tau}$ com ângulo $\vartheta \neq \pi / 2$.

Demonstração. Com respeito às coordenadas locais $(u, v)$ de $M$ definidas em (4.14), a Proposição 4.8 implica que o vetor posição de uma superfície de ângulo constante em $\mathbb{R}_{2}^{4}$ é dado por

$$
F(u, v)=\cos \left(\alpha_{1} u\right) g^{1}(v)+\operatorname{sen}\left(\alpha_{1} u\right) g^{2}(v)+\cos \left(\alpha_{2} u\right) g^{3}(v)+\operatorname{sen}\left(\alpha_{2} u\right) g^{4}(v)
$$

onde os vetores $\left\{g^{i}(v)\right\}_{i=1}^{4}$ são ortogonais entre si e

$$
\begin{gathered}
\left\|g^{1}(v)\right\|=\left\|g^{2}(v)\right\|=\sqrt{-g_{11}}=\text { constante }, \\
\left\|g^{3}(v)\right\|=\left\|g^{4}(v)\right\|=\sqrt{g_{33}}=\text { constante } .
\end{gathered}
$$

Portanto, se fizermos $e_{i}(v)=g^{i}(v) /\left\|g^{i}(v)\right\|, i \in\{1, \ldots, 4\}$, podemos escrever:

$$
\begin{aligned}
F(u, v)= & \sqrt{-g_{11}}\left(\cos \left(\alpha_{1} u\right) e_{1}(v)+\operatorname{sen}\left(\alpha_{1} u\right) e_{2}(v)\right) \\
& +\sqrt{g_{33}}\left(\cos \left(\alpha_{2} u\right) e_{3}(v)+\operatorname{sen}\left(\alpha_{2} u\right) e_{4}(v)\right) .
\end{aligned}
$$

Agora, as identidades (4.26), avaliadas em $(0, v)$, se tornam respectivamente:

$$
\begin{aligned}
& \alpha_{2} g_{33}\left\langle J_{1} e_{3}, e_{4}\right\rangle-\alpha_{1} g_{11}\left\langle J_{1} e_{1}, e_{2}\right\rangle \\
& +\sqrt{-g_{11} g_{33}}\left(\alpha_{1}\left\langle J_{1} e_{3}, e_{2}\right\rangle+\alpha_{2}\left\langle J_{1} e_{1}, e_{4}\right\rangle\right)=-\tau^{-1} \operatorname{sen}^{2} \vartheta \\
& \left\langle J_{1} e_{1}, e_{3}\right\rangle=0, \\
& \alpha_{2}^{3} g_{33}\left\langle J_{1} e_{3}, e_{4}\right\rangle-\alpha_{1}^{3} g_{11}\left\langle J_{1} e_{1}, e_{2}\right\rangle \\
& +\sqrt{-g_{11} g_{33}}\left(\alpha_{1} \alpha_{2}^{2}\left\langle J_{1} e_{3}, e_{2}\right\rangle+\alpha_{1}^{2} \alpha_{2}\left\langle J_{1} e_{1}, e_{4}\right\rangle\right)=-I,
\end{aligned}
$$




$$
\begin{gathered}
\left\langle J_{1} e_{2}, e_{4}\right\rangle=0, \\
\alpha_{1}\left\langle J_{1} e_{2}, e_{3}\right\rangle+\alpha_{2}\left\langle J_{1} e_{1}, e_{4}\right\rangle=0, \\
\alpha_{2}\left\langle J_{1} e_{2}, e_{3}\right\rangle+\alpha_{1}\left\langle J_{1} e_{1}, e_{4}\right\rangle=0 .
\end{gathered}
$$

Observamos que para obter as identidades acima fizemos divisões por $\alpha_{1}^{2}-\alpha_{2}^{2}=4 \tau^{-1} \sqrt{B^{3}} \cos \vartheta$ que é, por suposição em $\vartheta$, sempre diferente de zero. De (4.50) e (4.51), levando em conta que $\alpha_{1}^{2}-\alpha_{2}^{2} \neq 0$, resulta que

$$
\left\langle J_{1} e_{3}, e_{2}\right\rangle=0, \quad\left\langle J_{1} e_{1}, e_{4}\right\rangle=0 \text {. }
$$

\section{Portanto}

$$
\left|\left\langle J_{1} e_{1}, e_{2}\right\rangle\right|=1=\left|\left\langle J_{1} e_{3}, e_{4}\right\rangle\right|
$$

Substituindo (4.52) em (4.48) e (4.49), obtemos o sistema

$$
\left\{\begin{array}{l}
\alpha_{1} g_{11}\left\langle J_{1} e_{1}, e_{2}\right\rangle-\alpha_{2} g_{33}\left\langle J_{1} e_{3}, e_{4}\right\rangle=\tau^{-1} \operatorname{sen}^{2} \vartheta \\
\alpha_{1}^{3} g_{11}\left\langle J_{1} e_{1}, e_{2}\right\rangle-\alpha_{2}^{3} g_{33}\left\langle J_{1} e_{3}, e_{4}\right\rangle=I,
\end{array}\right.
$$

cuja solução é

$$
\left\langle J_{1} e_{1}, e_{2}\right\rangle=\frac{\tau I-\alpha_{2}^{2} \operatorname{sen}^{2} \vartheta}{\tau g_{11} \alpha_{1}\left(\alpha_{1}^{2}-\alpha_{2}^{2}\right)}, \quad\left\langle J_{1} e_{3}, e_{4}\right\rangle=\frac{\tau I-\alpha_{1}^{2} \operatorname{sen}^{2} \vartheta}{\tau g_{33} \alpha_{2}\left(\alpha_{1}^{2}-\alpha_{2}^{2}\right)}
$$

Agora, como

$$
g_{11} g_{33}=-\frac{\operatorname{sen}^{2} \vartheta}{4 B}, \quad \alpha_{1} \alpha_{2}=\frac{B}{\tau^{2}} \operatorname{sen}^{2} \vartheta, \quad \alpha_{1}^{2}-\alpha_{2}^{2}=\frac{4 \sqrt{B^{3}}}{\tau} \cos \vartheta
$$

resulta que

$$
\left\langle J_{1} e_{1}, e_{2}\right\rangle\left\langle J_{1} e_{3}, e_{4}\right\rangle=1 \text {. }
$$

Além disso, como

$$
\tau I-\alpha_{2}^{2} \operatorname{sen}^{2} \vartheta=2 \tau^{-1} \sqrt{B^{3}} \cos \vartheta \operatorname{sen}^{2} \vartheta
$$

resulta que $\left\langle J_{1} e_{1}, e_{2}\right\rangle<0$. Consequentemente,

$$
\left\langle J_{1} e_{1}, e_{2}\right\rangle=\left\langle J_{1} e_{3}, e_{4}\right\rangle=-1
$$


e $J_{1} e_{1}=e_{2}, J_{1} e_{3}=-e_{4}$.

Então, se fixarmos a base ortonormal de $\mathbb{R}_{2}^{4}$ dada por

$$
\tilde{E}_{1}=(0,0,1,0), \quad \tilde{E}_{2}=(0,0,0,1), \quad \tilde{E}_{3}=(1,0,0,0), \quad \tilde{E}_{4}=(0,-1,0,0),
$$

deve existir uma família a um parâmetro de matrizes $4 \times 4$ ortogonais indefinidas $A(v) \in \mathrm{O}_{2}(4)$, com $J_{1} A(v)=A(v) J_{1}$, tal que $e_{i}(v)=A(v) \tilde{E}_{i}$. Substituindo $e_{i}(v)=A(v) \tilde{E}_{i}$ em (4.47) obtemos

$$
F(u, v)=A(v) \gamma(u)
$$

onde

$$
\gamma(u)=\left(\sqrt{g_{33}} \cos \left(\alpha_{2} u\right),-\sqrt{g_{33}} \operatorname{sen}\left(\alpha_{2} u\right), \sqrt{-g_{11}} \cos \left(\alpha_{1} u\right), \sqrt{-g_{11}} \operatorname{sen}\left(\alpha_{1} u\right)\right)
$$

é uma curva em $\mathrm{SL}(2, \mathbb{R})_{\tau}$.

Examinemos agora a família a um parâmetro $A(v)$ que, de acordo com (4.29), depende de quatro funções $\xi_{1}(v), \xi_{2}(v), \xi_{3}(v)$ e $\xi(v)$.

Primeiro observamos que $\left\langle F_{v}, F_{v}\right\rangle=-\operatorname{sen}^{2} \vartheta=$ constante. De fato, usando (4.15) obtemos

$$
\left\langle F_{v}, F_{v}\right\rangle=a^{2}\left\langle F_{u}, F_{u}\right\rangle+2 a b\left\langle F_{u}, J F_{u}\right\rangle+b^{2}\left\langle J F_{u}, J F_{u}\right\rangle
$$

onde, de (4.25), temos

$$
\left\langle F_{u}, F_{u}\right\rangle=\widetilde{a}=-\tau^{-2} \operatorname{sen}^{2} \vartheta B
$$

Além disso, com auxílio de (1.3) e (4.25) obtemos $\left\langle F_{u}, J F_{u}\right\rangle=0$ e $\left\langle J F_{u}, J F_{u}\right\rangle=-\operatorname{sen}^{2} \vartheta$, uma vez que $\left\langle J F_{u}, J_{1} F_{u}\right\rangle=0$. Portanto, temos de fato $\left\langle F_{v}, F_{v}\right\rangle=-\operatorname{sen}^{2} \vartheta$.

Dessa forma, resulta

$$
\frac{\partial}{\partial u}\left\langle F_{v}, F_{v}\right\rangle_{\mid u=0}=0
$$

Agora, se denotarmos por $\mathbf{c}_{1}, \mathbf{c}_{2}, \mathbf{c}_{3}, \mathbf{c}_{4}$ as quatro colunas de $A(v)$, a equação (4.53) implica que

$$
\left\{\begin{array}{l}
\left\langle\mathbf{c}_{2}{ }^{\prime}, \mathbf{c}_{3}{ }^{\prime}\right\rangle=0, \\
\left\langle\mathbf{c}_{2}{ }^{\prime}, \mathbf{c}_{4}{ }^{\prime}\right\rangle=0,
\end{array}\right.
$$


onde com ' indicamos a derivação com respeito a $v$. Substituindo em (4.54) as expressões de $\mathbf{c}_{i}$ como funções de $\xi_{1}(v), \xi_{2}(v), \xi_{3}(v)$ e $\xi(v)$, obtemos

$$
\left\{\begin{array}{l}
\xi^{\prime} h(v)=0, \\
\xi^{\prime} k(v)=0,
\end{array}\right.
$$

onde $h(v)$ e $k(v)$ são duas funções tais que

$$
h^{2}+k^{2}=4\left(\xi_{1}^{\prime}\right)^{2}+\operatorname{senh}^{2}\left(2 \xi_{1}\right)\left(-\xi^{\prime}+\xi_{2}^{\prime}+\xi_{3}^{\prime}\right)^{2} .
$$

De (4.55) temos duas possibilidades:

(i) $\xi=$ constante;

$\mathrm{ou}$

(ii) $4\left(\xi_{1}^{\prime}\right)^{2}+\operatorname{senh}^{2}\left(2 \xi_{1}\right)\left(-\xi^{\prime}+\xi_{2}^{\prime}+\xi_{3}^{\prime}\right)^{2}=0$.

Mostraremos que o caso (ii) não pode ocorrer, mais precisamente, mostraremos que se valer (ii) então a parametrização $F(u, v)=A(v) \gamma(u)$ define um cilindro de Hopf, ou seja, o campo de Hopf $E_{1}$ é tangente à superfície. Para este fim, escrevemos o campo de vetores normal unitário $N$ como

$$
N=\frac{N_{1} E_{1}+N_{2} E_{2}+N_{3} E_{3}}{\sqrt{N_{1}^{2}+N_{2}^{2}+N_{3}^{2}}} .
$$

Um cálculo longo mas direto (que também pode ser feito com o auxílio de um software de cálculos simbólicos) nos dá

$$
\begin{aligned}
N_{1} & =1 / 2\left(\alpha_{1}+\alpha_{2}\right) \sqrt{-g_{11}} \sqrt{g_{33}}\left[2 \xi_{1}^{\prime} \cos \left(\alpha_{1} u+\alpha_{2} u-\xi_{2}+\xi_{3}\right)\right. \\
& \left.+\operatorname{senh}\left(2 \xi_{1}\right) \operatorname{sen}\left(\alpha_{1} u+\alpha_{2} u-\xi_{2}+\xi_{3}\right)\left(-\xi^{\prime}+\xi_{2}^{\prime}+\xi_{3}^{\prime}\right)\right] .
\end{aligned}
$$

Agora, o caso (ii) ocorre se, e somente se, $\xi_{1}=$ constante $=0$, ou se $\xi_{1}=$ constante $\neq 0$ e $-\xi^{\prime}+\xi_{2}^{\prime}+\xi_{3}^{\prime}=0$. Em ambos casos $N_{1}=0$, isso implica que

$$
g_{\tau}\left(N, J_{1} F\right)=-\tau g_{\tau}\left(N, E_{1}\right)=0,
$$


i.e. o campo de Hopf é tangente à superfície. Então concluímos que $\xi=$ constante.

Finalmente, neste caso, (4.28) é equivalente a equação:

$$
\tau \cos \vartheta \sqrt{B}\left[\cosh ^{2}\left(\xi_{1}(v)\right) \xi_{2}^{\prime}(v)+\operatorname{senh}^{2}\left(\xi_{1}(v)\right) \xi_{3}^{\prime}(v)\right]=0
$$

Como $\vartheta \neq \pi / 2$ concluímos que a condição (4.46) é satisfeita.

A volta do teorema segue imediatamente da Proposição 4.6, já que com cálculos diretos obte$\operatorname{mos} g_{\tau}\left(F_{u}, F_{u}\right)=g_{\tau}\left(E_{1}, F_{u}\right)=\operatorname{sen}^{2} \vartheta$, ou seja, (4.27) é satisfeita, enquanto que (4.46) é equivalente a equação (4.28).

\subsection{0 caso $B<0$}

Nesta seção estudaremos o caso em que a constante $B<0$. Integrando (4.21) temos o seguinte resultado:

Proposição 4.10. Seja $M^{2}$ uma superfície de ângulo constante em $\operatorname{SL}(2, \mathbb{R})_{\tau}$ com ângulo $\vartheta$ e $B<0$. Então, com respeito às coordenadas locais $(u, v)$ de $M$ definidas em (4.14), o vetor posição F de $M^{2}$ em $\mathbb{R}_{2}^{4}$ é dado por

$$
\begin{aligned}
F(u, v) & =\cos \left(\frac{\tilde{b}}{2} u\right)\left[\cosh (\beta u) w^{1}(v)+\operatorname{senh}(\beta u) w^{3}(v)\right] \\
& +\operatorname{sen}\left(\frac{\tilde{b}}{2} u\right)\left[\cosh (\beta u) w^{2}(v)+\operatorname{senh}(\beta u) w^{4}(v)\right],
\end{aligned}
$$

onde

$$
\beta=\sqrt{-B} \cos \vartheta
$$

é uma constante real, $\tilde{b}=-2 \tau^{-1} B$, enquanto que $w^{i}(v), i \in\{1, \ldots, 4\}$, são campos de vetores em $\mathbb{R}^{4}$, dependendo apenas de $v$, tais que

$$
\begin{aligned}
& \left\langle w^{1}(v), w^{1}(v)\right\rangle=\left\langle w^{2}(v), w^{2}(v)\right\rangle=-\left\langle w^{3}(v), w^{3}(v)\right\rangle=-\left\langle w^{4}(v), w^{4}(v)\right\rangle=1, \\
& \left\langle w^{1}(v), w^{2}(v)\right\rangle=\left\langle w^{1}(v), w^{3}(v)\right\rangle=\left\langle w^{2}(v), w^{4}(v)\right\rangle=\left\langle w^{3}(v), w^{4}(v)\right\rangle=0, \\
& \left\langle w^{1}(v), w^{4}(v)\right\rangle=-\left\langle w^{2}(v), w^{3}(v)\right\rangle=-\frac{2 \beta}{\tilde{b}} .
\end{aligned}
$$


Demonstração. Uma integração de (4.21), nos dá como solução

$$
\begin{aligned}
F(u, v) & =\cos \left(\frac{\tilde{b}}{2} u\right)\left[\cosh (\beta u) w^{1}(v)+\operatorname{senh}(\beta u) w^{3}(v)\right] \\
& +\operatorname{sen}\left(\frac{\tilde{b}}{2} u\right)\left[\cosh (\beta u) w^{2}(v)+\operatorname{senh}(\beta u) w^{4}(v)\right]
\end{aligned}
$$

onde

$$
\beta=\frac{\sqrt{4 \tilde{a}-\tilde{b}^{2}}}{2}=\sqrt{-B} \cos \vartheta
$$

é uma constante, enquanto que $w^{i}(v), i \in\{1, \ldots, 4\}$, são campos de vetores de $\mathbb{R}^{4}$ que dependem apenas de $v$. Se $w_{i j}(v):=\left\langle w^{i}(v), w^{j}(v)\right\rangle$, avaliando as relações (4.25) em $(0, v)$, obtemos

$$
\begin{aligned}
& w_{11}=1, \\
& \frac{\tilde{b}^{2}}{4} w_{22}+\beta^{2} w_{33}+\beta \tilde{b} w_{23}=\tilde{a}, \\
& \frac{\tilde{b}}{2} w_{12}+\beta w_{13}=0 \\
& \frac{\tilde{b}}{2}\left(\beta^{2}-\frac{\tilde{b}^{2}}{4}\right) w_{12}+\beta^{2} \tilde{b} w_{34}+\beta \frac{\tilde{b}^{2}}{2} w_{24}+\beta\left(\beta^{2}-\frac{\tilde{b}^{2}}{4}\right) w_{13}=0, \\
& \left(\beta^{2}-\frac{\tilde{b}^{2}}{4}\right)^{2} w_{11}+\beta^{2} \tilde{b}^{2} w_{44}++2 \beta \tilde{b}\left(\beta^{2}-\frac{\tilde{b}^{2}}{4}\right) w_{14}=D, \\
& \left(\beta^{2}-\frac{\tilde{b}^{2}}{4}\right) w_{11}+\beta \tilde{b} w_{14}=-\tilde{a}, \\
& \frac{\tilde{b}^{2}}{4}\left(3 \beta^{2}-\frac{\tilde{b}^{2}}{4}\right) w_{22}+\beta^{2}\left(\beta^{2}-3 \frac{\tilde{b}^{2}}{4}\right) w_{33}++\beta \frac{\tilde{b}}{2}\left(4 \beta^{2}-\tilde{b}^{2}\right) w_{23}=-D \text {, } \\
& \frac{\tilde{b}}{2}\left(3 \beta^{2}-\frac{\tilde{b}^{2}}{4}\right)\left(\beta^{2}-\frac{\tilde{b}^{2}}{4}\right) w_{12}+\tilde{b} \beta^{2}\left(\beta^{2}-3 \frac{\tilde{b}^{2}}{4}\right) w_{34} \\
& +\beta\left(\beta^{2}-3 \frac{\tilde{b}^{2}}{4}\right)\left(\beta^{2}-\frac{\tilde{b}^{2}}{4}\right) w_{13}+\beta \frac{\tilde{b}^{2}}{2}\left(3 \beta^{2}-\frac{\tilde{b}^{2}}{4}\right) w_{24}=0, \\
& \frac{\tilde{b}}{2}\left(3 \beta^{2}-\frac{\tilde{b}^{2}}{4}\right) w_{12}+\beta\left(\beta^{2}-3 \frac{\tilde{b}^{2}}{4}\right) w_{13}=0, \\
& \frac{\tilde{b}^{2}}{4}\left(3 \beta^{2}-\frac{\tilde{b}^{2}}{4}\right)^{2} w_{22}+\beta^{2}\left(\beta^{2}-3 \frac{\tilde{b}^{2}}{4}\right)^{2} w_{33} \\
& +\beta \tilde{b}\left(3 \beta^{2}-\frac{\tilde{b}^{2}}{4}\right)\left(\beta^{2}-3 \frac{\tilde{b}^{2}}{4}\right) w_{23}=E .
\end{aligned}
$$


De (4.57), 4.61) e (4.62), segue que

$$
w_{11}=-w_{44}=1, \quad w_{14}=-\frac{2 \beta}{\tilde{b}} .
$$

Além disso, de (4.59) e (4.65), obtemos

$$
w_{12}=w_{13}=0
$$

e, portanto, de (4.60) e (4.64),

$$
w_{24}=w_{34}=0 .
$$

Também, usando (4.58), (4.63) e (4.66), obtemos

$$
w_{22}=-w_{33}=1, \quad w_{23}=\frac{2 \beta}{\tilde{b}} .
$$

Teorema 4.11. Seja $M^{2}$ uma superfície de ângulo constante em $\operatorname{SL}(2, \mathbb{R})_{\tau}$ com ângulo $\vartheta \neq \pi / 2$ tal que $B<0$. Então, localmente, o vetor posição de $M^{2}$ em $\mathbb{R}_{2}^{4}$, com respeito às coordenadas locais $(u, v)$ de $M$ definidas em (4.14), é dado por

$$
F(u, v)=A(v) \gamma(u)
$$

onde a curva $\left.\gamma(u)=\left(\gamma_{1}(u), \gamma_{2}(u), \gamma_{3}(u), \gamma_{4}(u)\right)\right)$ é dada por

$$
\left\{\begin{array}{l}
\gamma_{1}(u)=\cos \left(\frac{\tilde{b}}{2} u\right) \cosh (\beta u)-\frac{2 \beta}{\tilde{b}} \operatorname{sen}\left(\frac{\tilde{b}}{2} u\right) \operatorname{senh}(\beta u), \\
\gamma_{2}(u)=\operatorname{sen}\left(\frac{\tilde{b}}{2} u\right) \cosh (\beta u)+\frac{2 \beta}{\tilde{b}} \cos \left(\frac{\tilde{b}}{2} u\right) \operatorname{senh}(\beta u), \\
\gamma_{3}(u)=\frac{\operatorname{sen} \vartheta}{\sqrt{-B}} \cos \left(\frac{\tilde{b}}{2} u\right) \operatorname{senh}(\beta u), \\
\gamma_{4}(u)=\frac{\operatorname{sen} \vartheta}{\sqrt{-B}} \operatorname{sen}\left(\frac{\tilde{b}}{2} u\right) \operatorname{senh}(\beta u),
\end{array}\right.
$$

$\beta=\sqrt{-B} \cos \vartheta, \tilde{b}=-2 \tau^{-1} B$ e $A(v)=A\left(\xi, \xi_{1}, \xi_{2}, \xi_{3}\right)(v)$ é uma família a um parâmetro de matrizes $4 \times 4$ ortogonais indefinidas que anti-comutam com $J_{1}$, como descrito em (4.29), com 
$\xi=$ constante $e$

$\operatorname{sen} \vartheta\left[2 \cos \left(\xi_{2}(v)-\xi_{3}(v)\right) \xi_{1}^{\prime}(v)+\left(\xi_{2}^{\prime}(v)+\xi_{3}^{\prime}(v)\right) \operatorname{sen}\left(\xi_{2}(v)-\xi_{3}(v)\right) \operatorname{senh}\left(2 \xi_{1}(v)\right)\right]$

$-2 \tau \cos \vartheta\left[\cosh ^{2}\left(\xi_{1}(v)\right) \xi_{2}^{\prime}(v)+\operatorname{senh}^{2}\left(\xi_{1}(v)\right) \xi_{3}^{\prime}(v)\right]=0$.

Reciprocamente, uma parametrização $F(u, v)=A(v) \gamma(u)$, com $\gamma(u)$ e A(v) como acima, define uma superfície de ângulo constante em $\operatorname{SL}(2, \mathbb{R})_{\tau}$ com ângulo $\vartheta \neq \pi / 2$.

Demonstração. De (4.56), podemos definir a seguinte base pseudo-ortonormal em $\mathbb{R}_{2}^{4}$ :

$$
\left\{\begin{array}{l}
e_{1}(v)=w^{1}(v), \\
e_{2}(v)=w^{2}(v), \\
e_{3}(v)=\frac{1}{\operatorname{sen} \vartheta}\left[\sqrt{-B} w^{3}(v)-\tau \cos \vartheta w^{2}(v)\right], \\
e_{4}(v)=\frac{1}{\operatorname{sen} \vartheta}\left[\sqrt{-B} w^{4}(v)+\tau \cos \vartheta w^{1}(v)\right],
\end{array}\right.
$$

Onde $\left\langle e_{1}, e_{1}\right\rangle=1=\left\langle e_{2}, e_{2}\right\rangle$ e $\left\langle e_{3}, e_{3}\right\rangle=-1=\left\langle e_{4}, e_{4}\right\rangle$.

Avaliando as identidades (4.26) em $(0, v)$, e levando em conta que:

$$
\begin{aligned}
& F(0, v)=w^{1}(v) \\
& F_{u}(0, v)=\frac{\tilde{b}}{2} w^{2}(v)+\beta w^{3}(v) \\
& F_{\text {uи }}(0, v)=\left(\beta^{2}-\frac{\tilde{b}^{2}}{4}\right) w^{1}(v)+\beta \tilde{b} w^{4}(v) \\
& F_{\text {uиu }}(0, v)=\frac{\tilde{b}}{2}\left(3 \beta^{2}-\frac{\tilde{b}^{2}}{4}\right) w^{2}(v)+\beta\left(\beta^{2}-\frac{3}{4} \tilde{b}^{2}\right) w^{3}(v), \\
& F_{\text {uииu }}(0, v)=\left(\beta^{4}-\frac{3}{2} \beta^{2} \tilde{b}^{2}+\frac{\tilde{b}^{4}}{16}\right) w^{1}(v)+2 \beta \tilde{b}\left(\beta^{2}-\frac{\tilde{b}^{2}}{4}\right) w^{4}(v),
\end{aligned}
$$

concluímos que

$$
\begin{aligned}
& \left\langle J_{1} w^{3}, w^{4}\right\rangle=-\left\langle J_{1} w^{1}, w^{2}\right\rangle=1, \\
& \left\langle J_{1} w^{3}, w^{2}\right\rangle=\left\langle J_{1} w^{1}, w^{4}\right\rangle=0, \\
& \left\langle J_{1} w^{2}, w^{4}\right\rangle=\left\langle J_{1} w^{1}, w^{3}\right\rangle=-\frac{\tau \cos \vartheta}{\sqrt{-B}} .
\end{aligned}
$$

Então,

$$
-\left\langle J_{1} e_{1}, e_{2}\right\rangle=\left\langle J_{1} e_{3}, e_{4}\right\rangle=1
$$




$$
\left\langle J_{1} e_{1}, e_{4}\right\rangle=\left\langle J_{1} e_{1}, e_{3}\right\rangle=\left\langle J_{1} e_{2}, e_{3}\right\rangle=\left\langle J_{1} e_{2}, e_{4}\right\rangle=0
$$

Dessa forma, obtemos

$$
J_{1} e_{1}=-e_{2}, \quad J_{1} e_{3}=-e_{4}
$$

Consequentemente, se considerarmos a base pseudo-ortonormal $\left\{\hat{E}_{i}\right\}_{i=1}^{4}$ de $\mathbb{R}_{2}^{4}$ dada por

$$
\hat{E}_{1}=(1,0,0,0), \quad \hat{E}_{2}=(0,1,0,0), \quad \hat{E}_{3}=(0,0,1,0), \quad \hat{E}_{4}=(0,0,0,1),
$$

deve haver uma família a um parâmetro de matrizes $A(v) \in \mathrm{O}_{2}(4)$, com $J_{1} A(v)=-A(v) J_{1}$, tal que $e_{i}(v)=A(v) \hat{E}_{i}, i \in\{1, \ldots, 4\}$. Como

$$
F=\left\langle F, e_{1}\right\rangle e_{1}+\left\langle F, e_{2}\right\rangle e_{2}-\left\langle F, e_{3}\right\rangle e_{3}-\left\langle F, e_{4}\right\rangle e_{4},
$$

calculando $\left\langle F, e_{i}\right\rangle$ e substituindo $e_{i}(v)=A(v) \hat{E}_{i}$, obtemos que $F(u, v)=A(v) \gamma(u)$, onde a curva $\gamma(u)$ de $\operatorname{SL}(2, \mathbb{R})_{\tau}$ é dada em 4.67).

Examinemos agora a família a um parâmetro $A(v)$ que, de acordo com (4.29), depende de quatro funções $\xi_{1}(v), \xi_{2}(v), \xi_{3}(v)$ e $\xi(v)$. Analogamente ao que foi feito na prova do Teorema 4.9. temos que

$$
\frac{\partial}{\partial u}\left\langle F_{v}, F_{v}\right\rangle_{\mid u=0}=0
$$

implica que as funções $\xi_{1}(v), \xi_{2}(v), \xi_{3}(v)$ e $\xi(v)$ satisfazem a equação

$$
\xi^{\prime}\left[2 \operatorname{sen}\left(\xi_{2}-\xi_{3}\right) \xi_{1}^{\prime}-\left(\xi_{2}^{\prime}+\xi_{3}^{\prime}-\xi^{\prime}\right) \cos \left(\xi_{2}-\xi_{3}\right) \operatorname{senh}\left(2 \xi_{1}\right)\right]=0 .
$$

Então temos duas possibilidades:

(i) $\xi=$ constante;

ou

(ii) $2 \operatorname{sen}\left(\xi_{2}-\xi_{3}\right) \xi_{1}^{\prime}-\left(\xi_{2}^{\prime}+\xi_{3}^{\prime}-\xi^{\prime}\right) \cos \left(\xi_{2}-\xi_{3}\right) \operatorname{senh}\left(2 \xi_{1}\right)=0$. 
Também neste caso, usando os mesmos argumentos que no Teorema 4.9, vemos que a condição (ii) implica que a superfície seja um cilindro de Hopf, portanto podemos assumir que $\xi=$ constante. Finalmente, um cálculo longo mostra que, no caso em que $\xi=$ constante, (4.28) é equivalente (4.68).

A volta do teorema segue imediatamente da Proposição 4.6 já que, um cálculo direto confere $g_{\tau}\left(F_{u}, F_{u}\right)=g_{\tau}\left(E_{1}, F_{u}\right)=\operatorname{sen}^{2} \vartheta$, ou seja, vale (4.27) enquanto (4.68) é equivalente a (4.28). 

[1] G.B. Airy. On the strains in the interior of beams. Philos. Trans. R. Soc. London Ser. A, 153 (1863), 49-79.

[2] A. Back, M.P. do Carmo, W.Y. Hsiang. On some fundamental equations of equivariant Riemannian geometry. Tamkang J. Math. 40 (2009), no. 4, 343-376.

[3] P. Baird, J. Eells. A conservation law for harmonic maps. Geometry Symposium Utrecht 1980, 1-25, Lecture Notes in Math., 894, Springer 1981.

[4] A. Balmuş. On the biharmonic curves of the Euclidean and Berger 3-dimensional spheres. Sci. Ann. Univ. Agric. Sci. Vet. Med. 47 (2004), 87-96.

[5] A. Balmuş, S. Montaldo, C. Oniciuc. Biharmonic PNMC submanifolds in sphere. Ark. Mat. 51 (2013), 1-16.

[6] A. Balmuş, S. Montaldo, C. Oniciuc. Submanifolds with biharmonic Gauss map. Internat. J. Math. 21 (2010), no. 12, 1585-163.

[7] L. Bianchi. Gruppi continui e finiti. Ed. Zanichelli, Bologna (1928). 
[8] R. Caddeo, S. Montaldo, C. Oniciuc. Biharmonic submanifolds of $\mathbb{S}^{3}$. Internat. J. Math. 12 (2001), 867-876.

[9] R. Caddeo, S. Montaldo, C. Oniciuc. Biharmonic submanifolds in spheres. Israel J. Math. 130 (2002), 109-123.

[10] R. Caddeo, S. Montaldo, C. Oniciuc, P. Piu. Surfaces in three-dimensional space forms with divergence-free stress-bienergy tensor. Ann. Mat. Pura Appl. Volume 193 (2014), $529-550$.

[11] R. Caddeo, S. Montaldo, C. Oniciuc, P. Piu. The Euler-Lagrange method for biharmonic curves. Mediterr. J. Math. 3 (2006), no. 3-4, 449-465.

[12] R. Caddeo, S. Montaldo, P. Piu. Biharmonic curves on a surface. Rendiconti di Matematica e delle sue Applicazioni Serie VII, Volume 21, Roma (2001), 143-157.

[13] R. Caddeo, P. Piu, C. Oniciuc. Explicit formulas for non-geodesic biharmonic curves of the Heisenberg group. Ren. Mat. Univ. Politc. Torino 62 (2004), no. 3, 265-277.

[14] R. Caddeo, L. Vanhecke. Does " $\Delta^{2} d^{2-n}=0$ on a Riemannian manifold" implies flatness? Period. Math. Hungar. 17 (1986), 109-117.

[15] É. Cartan. Leçons sur la géométrie des espaces de Riemann. Gauthier Villars, Paris (1946).

[16] P. Cermelli, A. J. Di Scala. Constant-angle surfaces in liquid crystals. Phil. Mag. 87 (2007), 1871-1888.

[17] B-Y. Chen. Total Mean Curvature and Submanifolds of finite Type. Series in Pure Mathematics 1. World Scintific Publishing Co., Singapore, 1884.

[18] B. Daniel. Isometric immersions into 3-dimensional homogeneous manifolds. Comment. Math. Helv. 82, (2007), 87-131. 
[19] M. Dajczer, J. H. de Lira. Killing graphs with prescribed mean curvature and Riemannian submersions. Ann. Ins. H. Poincaré Anal. Non Linéaire 26 (2009), no. 3, 763-775.

[20] F. Dillen, M.I. Munteanu. Constant angle surfaces in $\mathbb{H}^{2} \times \mathbb{R}$. Bull. Braz. Math. Soc. (N.S.) 40 (2009), 85-97.

[21] F. Dillen, J. Fastenakels, J. Van der Veken, L. Vrancken. Constant angle surfaces in $\mathbb{S}^{2} \times \mathbb{R}$. Monatsh. Math. 152 (2007), 89-96.

[22] L. Sario, M. Nakai, C. Wang, L. Chung. Classification theory of Riemannian manifolds. Harmonic, quasiharmonic and biharmonic functions. Lecture Notes in Mathematics, Vol. 605. Springer-Verlag, Berlin-New York, 1977.

[23] P.G. de Gennes, J. Prost. The Physics of Liquid Crystals, 2nd edn. Clarendon Press, Oxford (1993).

[24] A. Di Scala, G. Ruiz-Hernández. Higher codimensional Euclidean helix submanifolds. Kodai Math. J. 33 (2010), 192-210.

[25] A. Di Scala, G. Ruiz-Hernández. Helix submanifolds of Euclidean spaces. Monatsh. Math. 157 (2009), 205-215.

[26] I. Dimitric. Submanifolds of $\mathbb{E}^{m}$ with harmonic mean curvature vetor. Bull. Inst. Math. Acad. Sinica 20 (1992), 53-65.

[27] M.P. do Carmo. Geometria Riemanniana. Projeto Euclides, 10. Instituto de Matemática Pura e Aplicada, Rio de Janeiro, 1979.

[28] J. Eells and L. Lemaire. Selected topics in harmonic maps. Conf. Board Math. Sci. 50 (1983).

[29] J. Eells, J. H. Sampson. Harmonic mappings of Riemannian manifolds. Amer. J. Math. 86 (1964), 109-160. 
[30] J. M. Espinar. Finite index operators on surfaces. J. Geom. Anal. 23 (2013), no. 1, 415437.

[31] J. M. Espinar, I. S. de Oliveira. Locally convex surfaces immersed in a Killing submersion. Bull. Braz. Math. soc. (N.S) 44 (2013), no. 1, 155-171.

[32] J. M. Espinar, H. Rosemberg. When strictly locally convex hypersurfaces are embedded. Math. Z. 271 (2012), no. 3-4, 1075-1090.

[33] J. Fastenakels, M.I. Munteanu, J. Van Der Veken. Constant angle surfaces in the Heisenberg group. Acta Math. Sin. (Engl. Ser.) 27 (2011), 747-756.

[34] D. Fetcu, C. Oniciuc, A. L. Pinheiro. CMC biconservative surfaces in $\mathbb{S}^{n} \times \mathbb{R}$ and $\mathbb{H}^{n} \times \mathbb{R}$. J. Math. Anal. Appl. 425 (2015), no. 1, 588-609.

[35] G. Fubini, Sugli spazi che ammettono un gruppo continuo di movimenti. Ann. di Matem. Tomo 8, serie III, (1903), 39-82.

[36] D. Hilbert. Die grundlagen der physik. Math. Ann. 92 (1924), 1-32.

[37] W.T. Hsiang, W.Y. Hsiang. On the existence of codimension one minimal spheres in compact symmetric spaces of rank 2, II. J. Diff. Geom. 17 (1982), 583-594.

[38] W.T. Hsiang, H.B. Lawson Jr. Minimal Submanifold of low cohomogeneity. J. Diff. Geom. 5 (1971), 1-38.

[39] G.Y Jiang. 2-harmonic isometric immersions between Riemannian manifolds. Chinese Ann. Math. Ser A 7 (1986), no. 2, 130-144.

[40] G.Y Jiang. 2-harmonic maps and their first and second variational formulas. Chinese Ann. Math. Ser A 7 (1986), no. 4, 389-402.

[41] G.Y Jiang. The conservative law for 2-harmonic maps between Riemannian manifolds. Acta. Math. Sinica 30 (1987), 220-225. 
[42] S. Kobayashi, K. Nomizu. Foundations of Differential Geometry I/II. Tracts in Mathematics 15, Interscience, New York (1963).

[43] R. López, M.I. Munteanu. On the geometry of constant angle surfaces in $\mathrm{Sol}_{3}$. Kyushu J. Math. 65 (2011), 237-249.

[44] E. Loubeau, S. Montaldo. Biminimal imersions. Proc. Edinb. Math. Soc. 51 (2008), $421-437$.

[45] E. Loubeau, S. Montaldo, C. Oniciuc.The stress-energy tensor for biharmonic maps. Math. Z. 259 (2008), 503-524.

[46] J. M. Manzano. On the classification of Killing submersions and their isometries. Pacific J. Math. 270 (2014), no. 2, 367-392.

[47] J.C. Maxwell. On reciprocal diagrams in space, and their relation to Airy's function of stress. Proc. London. Math. Soc. 2 (1868), 102-105.

[48] M. A. Meroño, I. Ortiz. First stability eingenvalue characterization of CMC Hopf tori Riemannian Killing submersions. J. Math. Anal. Appl. 417 (2014), no. 1, 400-410.

[49] S. Montaldo, C. Oniciuc. A short survey on biharmonic maps between Riemannian manifolds. Rev. Un. Mat. Argentina 47 (2006), 1-22.

[50] S. Montaldo, C. Oniciuc, A. Ratto. Proper biconservative immersions into the Euclidean space. Ann. Mat. Pura Appl., DOI: 10.1007/s10231-014-0469-4.

[51] S. Montaldo, I.I. Onnis. Helix surfaces in the Berger sphere. Israel J. of Math. 201 (2014), no.2, 949-966.

[52] S. Montaldo, I.I. Onnis, A.P. Passamani. Helix surfaces in the special linear group. Ann. Mat. Pura Appl., DOI: 10.1007/s10231-014-0452-0. 
[53] S. Myers, N. Steenrod. The group of isometries of a Riemannian manifold. Ann. of Math. 40 (1939).

[54] M.I. Munteanu, A. Nistor. A new approach on constant angle surface in $\mathbb{E}^{3}$. Turkish J. Math. 33 (2009), no. 2, 169-178.

[55] P.J. Olver. Aplication of Lie Groups to Differential Equations. GTM 170, Springer Verlag, New York (1986).

[56] I.I. Onnis, A.P. Passamani. On the biharmonic curves in the special linear group $S L(2, \mathbb{R})$. Mediterr. J. Math., DOI: 10.1007/s00009-014-0474-2.

[57] Y.-L. Ou. Biharmonic hypersurfaces in Riemannian manifolds. Pacific J. Math. 248 (2010), 217-232.

[58] Y.-L Ou, Z.-P. Wang. Constant mean curvature and totally umbilical biharmonic surfaces in 3-dimensional geometries. J. Geom. Phys. 61 (2011), no. 10, 1845-1853.

[59] C. Oniciuc. Biharmonic maps between Riemannian manifolds. An. Stiint. Univ. Al.I. Cusa Iasi Mat (N.S) 48 (2002), 237-248.

[60] R.S. Palais. On the existence of slices for actions of non-compact Lie groups. Ann. of Math. 73 (1961), 296-323.

[61] R.S. Palais, C.L. Terng. Critical Point Theory and Submanifold Theory. LNM 1353, Springer-Verlag, New York (1980).

[62] M.P. Piu. Sur certains types de distributions non-integrables totalement geodesiques. Thèse de Doctorat, Université de Haute-Alsace (1988).

[63] H. Rosemberg, R. Souam, E. Toubiana. General curvature estimates for stable $H$ surfaces in 3-manifolds and applications. J. Differential Gom. 84 (2010), no. 3, 623648. 
[64] E. Ruh, J. Vilms. The tension field of the Gauss map. Trans. Amer. Math. Soc. 149 (1970), 569-573.

[65] G. Ruiz-Hernández. Minimal helix surfaces in $N^{n} \times \mathbb{R}$. Abh. Math. Semin. Univ. Hambg. $81(2011), 55-67$.

[66] A. Sanini. Applicazioni tra varietà riemanniane con energia critica rispetto a deformazioni di metriche. Rend. Mat. 3 (1983), 53-63.

[67] G. Vranceanu. Leçons de géométrie differentielle. Ed. Acad. Rep. Pop. Roum., vol. I, Bucarest (1957).

[68] Yu Fu. On bi-conservative surfaces in Minkowski 3-spaces. J. Geom. Phys. 66 (2013), $71-79$. 